\title{
Advances in atomic force microscopy
}

\author{
Franz J. Giessibl* \\ Experimentalphysik VI, Electronic Correlations and Magnetism, Institute of Physics, \\ Augsburg University, D-86135 Augsburg, Germany
}

(Published 29 July 2003)

\begin{abstract}
This article reviews the progress of atomic force microscopy in ultrahigh vacuum, starting with its invention and covering most of the recent developments. Today, dynamic force microscopy allows us to image surfaces of conductors and insulators in vacuum with atomic resolution. The most widely used technique for atomic-resolution force microscopy in vacuum is frequency-modulation atomic force microscopy (FM-AFM). This technique, as well as other dynamic methods, is explained in detail in this article. In the last few years many groups have expanded the empirical knowledge and deepened our theoretical understanding of frequency-modulation atomic force microscopy. Consequently spatial resolution and ease of use have been increased dramatically. Vacuum atomic force microscopy opens up new classes of experiments, ranging from imaging of insulators with true atomic resolution to the measurement of forces between individual atoms.
\end{abstract}

\section{CONTENTS}

I. Introduction

II. Principle of Atomic Force Microscopy

A. Relation to scanning tunneling microscopy

1. Tunneling current in scanning tunneling microscopy

2. Experimental measurement and noise

B. Tip-sample forces $F_{t s}$

C. The force sensor (cantilever)

1. Cantilever tips

2. Measurement of cantilever deflection and noise

3. Thermal stability

D. Operating modes of AFM's

1. Static atomic force microscopy

2. Dynamic atomic force microscopy

III. Challenges Faced by Atomic Force Microscopy with Respect to Scanning Tunneling Microscopy

A. Stability

B. Nonmonotonic imaging signal

C. Contribution of long-range forces

D. Noise in the imaging signal

IV. Early AFM Experiments

V. The Rush for Silicon

VI. Frequency-Modulation Atomic Force Microscopy

A. Experimental setup

B. Experimental parameters

VII. Physical Observables in FM-AFM

A. Frequency shift and conservative forces

1. Generic calculation

2. An intuitive expression for frequency shifts as a function of amplitude

3. Frequency shift for a typical tip-sample force

4. Deconvolution of forces from frequency shifts

B. Average tunneling current for oscillating tips

C. Damping and dissipative forces

VIII. Noise in Frequency-Modulation Atomic Force Microscopy

\footnotetext{
*Electronic address: Franz.Giessibl@physik.uni-augsburg.de
}

A. Generic calculation $\quad 970$

B. Noise in the frequency measurement 971

C. Optimal amplitude for minimal vertical noise 972

IX. Applications of Classic Frequency-Modulation

Atomic Force Microscopy 972

A. Imaging 972

B. Spectroscopy 973

X. New Developments 974

A. Dissipation measurements and theory 974

B. Off-resonance technique with small amplitudes $\quad 974$

C. Dynamic mode with stiff cantilevers and small amplitudes

D. Dynamic lateral force microscopy 976

XI. Summary and Conclusions 977

XII. Outlook 977

Acknowledgments 977

$\begin{array}{ll}\text { References } & 978\end{array}$

\section{INTRODUCTION}

Imaging individual atoms was an elusive goal until the introduction of the scanning tunneling microscope (STM) in 1981 by Binnig, Rohrer, Gerber, and Weibel (1982). This humble instrument has provided a breakthrough in our ability to investigate matter on the atomic scale: for the first time, the individual surface atoms of flat samples could be made visible in real space. Within one year of its invention, the STM helped to solve one of the most intriguing problems in surface science: the structure of the $\operatorname{Si}(111)-(7 \times 7)$ surface. The adatom layer of $\mathrm{Si}(111)-(7 \times 7)$ was imaged with an STM by Binnig et al. (1983). This image, combined with x-ray-scattering and electron-scattering data helped Takayanagi, Tanishiro, Takahashi, and Takahashi (1985) to develop the dimer-adatom-stacking fault (DAS) model for $\mathrm{Si}(111)-(7 \times 7)$. G. Binnig and H. Rohrer, the inventors of the STM, were rewarded with the Nobel Prize in Physics in 1986. The historic initial steps and the rapid success of the STM, including the resolution of the silicon $7 \times 7$ reconstruction, were described in their No- 


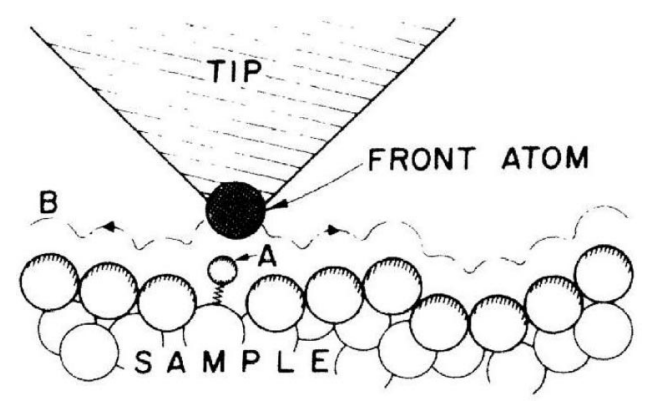

FIG. 1. Scanning tunneling microscope (STM) or atomic force microscope (AFM) tip close to a sample [Fig. 1(a) of Binnig et al. (1986)].

bel Prize lecture (1987). The spectacular spatial resolution of the STM along with its intriguing simplicity launched a broad research effort with a significant impact on surface science (Mody, 2002). A large number of metals and semiconductors have been investigated on the atomic scale and marvelous images of the world of atoms were created within the first few years after the inception of the STM. Today, the STM is an invaluable asset in the surface scientist's toolbox.

Despite the phenomenal success of the STM, it has a serious limitation. It requires electrical conduction of the sample material, because it uses the tunneling current which flows between a biased tip and a sample. However, early STM experiments showed that whenever the tip-sample distance was small enough that a current could flow, significant forces would act collaterally with the tunneling current. Soon it was speculated that these forces could be put to good use in the atomic force microscope (AFM). The force microscope was invented by Binnig (1986) and, shortly after its invention, Binnig, Quate, and Gerber (1986) introduced a working prototype, while Binnig and Gerber spent a sabbatical at Stanford and the IBM Research Laboratory in Almaden, California (Riordon, 2003). Binnig et al. (1986) were aware that, even during STM operation, significant forces between single atoms are acting, and they were confident that the AFM could ultimately achieve true atomic resolution (see Fig. 1, adapted from Binnig et al., 1986). The STM can only image electrically conductive samples, which limits its application to the imaging of metals and semiconductors. But even conductorsexcept for a few special materials, like highly oriented pyrolytic graphite (HOPG) — cannot be studied in ambient conditions by STM but have to be investigated in an ultrahigh vacuum. In ambient conditions, the surface layer of solids constantly changes by adsorption and desorption of atoms and molecules. An ultrahigh vacuum is required for clean and well-defined surfaces. Because electrical conductivity of the sample is not required in atomic force microscopy the AFM can image virtually any flat solid surface without the need for surface preparation. Consequently, thousands of AFM's are in use in university, public, and industrial research laboratories all over the world. Most of these instruments are operated in ambient conditions.
For studying surfaces on the atomic level, an ultrahigh-vacuum environment is required, where it is more difficult to operate an AFM. In addition to the experimental challenges of the STM, the AFM faces four more substantial experimental complications, which are summarized in Sec. III. While Binnig, Quate, and Gerber (1986) anticipated the true atomic resolution capability of the AFM from the beginning, it took five years before atomic resolution on inert surfaces could be demonstrated (Giessibl, 1991; Giessibl and Binnig, 1992b; Ohnesorge and Binnig, 1993; see Sec. IV). Resolving reactive surfaces by AFM with atomic resolution took almost a decade from the invention of the AFM. The Si(111)- $(7 \times 7)$ surface, a touchstone of the AFM's feasibility as a tool for surface science, was resolved with atomic resolution by dynamic atomic force microscopy (Giessibl, 1995). The new microscopy mode has proven to work as a standard method, and in 1997 Seizo Morita from Osaka University in Japan initiated an international workshop on the subject of "noncontact atomic force microscopy." A year later, the "First International Workshop on Non-contact Atomic Force Microscopy (NC-AFM)" was held in Osaka, Japan with about 80 attendees. This meeting was followed in 1999 by one in Pontresina (Switzerland) with roughly 120 participants and the "Third International Conference on Noncontact Atomic Force Microscopy (NC-AFM)" in Hamburg, Germany in 2000 with more than 200 participants. A fourth meeting took place in September 2001 in Kyoto, Japan, and the 2002 conference met at McGill University in Montreal, Canada. The next meeting is scheduled for Ireland in Summer 2003. The proceedings for these workshops and conferences (Morita and Tsukada, 1999; Bennewitz, Pfeiffer, et al., 2000; Schwarz et al., 2001; Tsukada and Morita, 2002; Hoffmann, 2003) and a recent review by Garcia and Perez (2002) are a rich source of information about atomic force microscopy and its role in surface science. Also, a multiauthor book about NC-AFM has recently become available (Morita et al., 2002). The introduction of this book (Morita, 2002) covers interesting aspects of the history of the AFM. This review can only cover a part of the field, and the author must apologize to the colleagues whose work he was not able to treat in the depth it deserved. However, many of these publications are listed in the bibliography and references therein.

\section{PRINCIPLE OF ATOMIC FORCE MICROSCOPY}

\section{A. Relation to scanning tunneling microscopy}

The AFM is closely related to the STM, and it shares its key components, except for the probe tip. The principle of the STM is explained very well in many excellent books and review articles, e.g., those of Binnig and Rohrer (1985, 1987, 1999); Güntherodt and Wiesendanger (1991); Chen (1993); Stroscio and Kaiser (1994); and Wiesendanger $(1994,1998)$. Nevertheless, the key principle of the STM is described here because the additional challenges faced by the AFM become apparent 


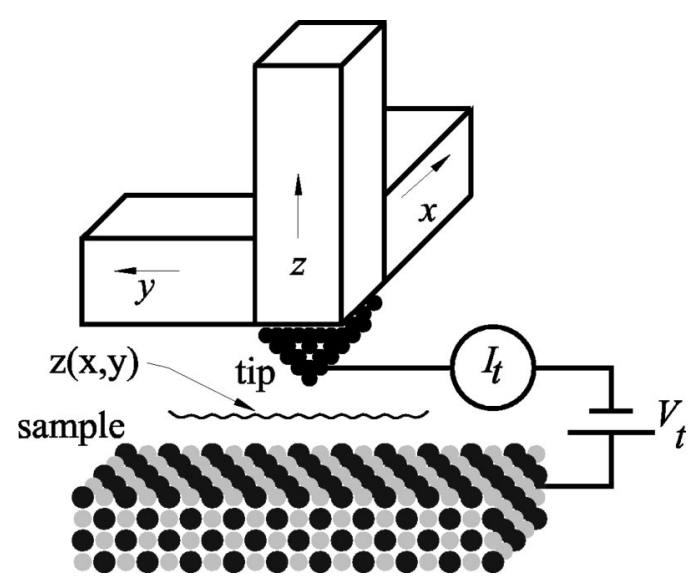

FIG. 2. A scanning tunneling microscope (schematic).

clearly in a direct comparison. Figure 2 shows the general setup of a scanning tunneling microscope (STM): a sharp tip is mounted on a scanning device known as an $x y z$ scanner, which allows three-dimensional positioning in the $x, y$, and $z$ directions with subatomic precision. The tunneling tip is typically a wire that has been sharpened by chemical etching or mechanical grinding. W, PtIr, or pure Ir are often chosen as the tip material. A bias voltage $V_{t}$ is applied to the sample, and when the distance between tip and sample is in the range of several angstroms, a tunneling current $I_{t}$ flows between the tip and sample. This current is used as the feedback signal in a $z$-feedback loop.

In the topographic mode, images are created by scanning the tip in the $x y$ plane and recording the $z$ position required to keep $I_{t}$ constant. In the constant-height mode, the probe scans rapidly so that the feedback cannot follow the atomic corrugations. The atoms are then apparent as modulations of $I_{t}$, which are recorded as a function of $x$ and $y$. The scanning is usually performed in a raster fashion with a fast scanning direction (sawtooth or sinusoidal signal) and a slow scanning direction (sawtooth signal). A computer controls the scanning of the surface in the $x y$ plane while recording the $z$ position of the tip (topographic mode) or $I_{t}$ (constant-height mode). Thus a three-dimensional image $z\left(x, y, I_{t}\right.$ $\approx$ const $)$ or $I_{t}(x, y, z \approx$ const $)$ is created.

In the AFM, the tunneling tip is replaced by a forcesensing cantilever. The tunneling tip can also be replaced by an optical near-field probe, a microthermometer etc., giving rise to a whole family of scanning probe microscopes (see Wickramasinghe, 1989).

\section{Tunneling current in scanning tunneling microscopy}

In an STM, a sharp tip is brought close to an electrically conductive surface that is biased at a voltage $V_{t}$. When the separation is small enough, a current $I_{t}$ flows between them. The typical distance between tip and sample under these conditions is a few atomic diameters, and the transport of electrons occurs by tunneling. When $\left|V_{t}\right|$ is small compared to the work function $\Phi$, the tunneling barrier is roughly rectangular (see Fig. 3) with

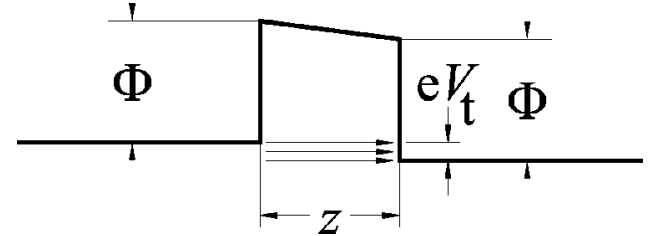

FIG. 3. Energy diagram of an idealized tunneling gap. The image charge effect (see Chen, 1993) is not taken into account here.

a width $z$ and a height given by the work function $\Phi$. According to elementary quantum mechanics, the tunneling current is given by

$$
I_{t}(z)=I_{0} e^{-2 \kappa_{t} z} .
$$

$I_{0}$ is a function of the applied voltage and the density of states in both tip and sample and

$$
\kappa_{t}=\sqrt{2 m \Phi} / \hbar,
$$

where $m$ is the mass of the electron and $\hbar$ is Planck's constant. For metals, $\Phi \approx 4 \mathrm{eV}$, thus $\kappa_{t} \approx 1 \AA^{-1}$. When $z$ is increased by one angstrom, the current drops by an order of magnitude. This strong distance dependence is pivotal for the atomic resolution capability of the STM. Most of the tunneling current is carried by the atom that is closest to the sample (the "front atom"). If the sample is very flat, this front atom remains the atom that is closest to the sample during scanning in $x$ and $y$, and even relatively blunt tips yield atomic resolution easily.

\section{Experimental measurement and noise}

The tunneling current is measured with a current-tovoltage converter (see Fig. 4), a simple form of which consists merely of a single operational amplifier (OPA) with low noise and low input bias current, and a feedback resistor with a typical impedance of $R=100 \mathrm{M} \Omega$ and small parasitic capacitance. The tunneling current $I_{t}$ is used to measure the distance between tip and sample. The noise in the imaging signal (the tunneling current in an STM, force or some derived quantity in an AFM) needs to be small enough that the corresponding vertical noise $\delta z$ is considerably smaller than the atomic corrugation of the sample. In the following, the noise levels

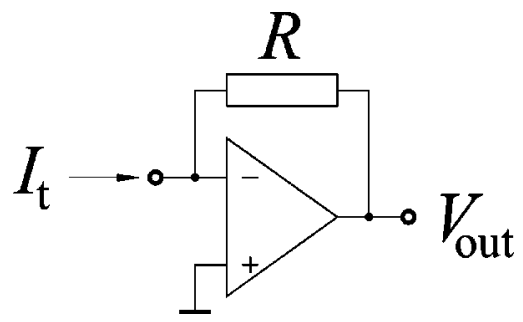

FIG. 4. A simple current-to-voltage converter for an STM and for the qPlus sensor shown in Fig. 11. It consists of an operational amplifier with high speed, low noise, and low input bias current, as well as a feedback resistor (typical impedance $R$ $\approx 10^{8} \Omega$ ) that has low parasitic capacitance. The output voltage is given by $V_{\text {out }}=-R \times I_{t}$. 


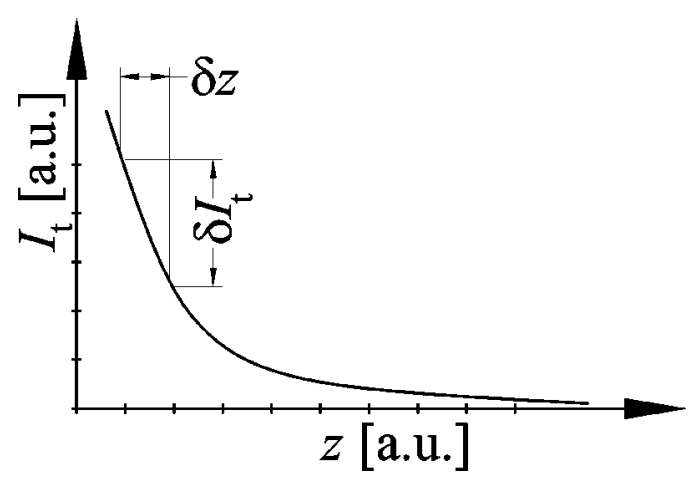

FIG. 5. Tunneling current as a function of distance and relation between current noise $\delta I_{t}$ and vertical noise $\delta z$ (arbitrary units).

for imaging signals and vertical positions are described by the root-mean-square (rms) deviation of the mean value and indicated by the prefix $\delta$, i.e.,

$$
\delta \xi \equiv \sqrt{\left\langle(\xi-\langle\xi\rangle)^{2}\right\rangle}
$$

To achieve atomic resolution with an STM or AFM, a first necessary condition is that the mechanical vibrations between tip and sample be smaller than the atomic corrugations. This condition is met by a microscope design emphasizing utmost stability and establishing proper vibration isolation, such as is described by Kuk and Silverman (1988); Chen (1993); or Park and Barrett (1993). In the following, proper mechanical design and vibration isolation will be presumed and are not discussed further. The inherent vertical noise in an STM is connected to the noise in the current measurement. Figure 5 shows the qualitative dependence of the tunneling current $I_{t}$ on vertical distance $z$. Because the measurement of $I_{t}$ is subject to noise, the vertical distance measurement is also subject to a noise level $\delta z$ :

$$
\delta z_{I_{t}}=\frac{\delta I_{t}}{\left|\frac{\partial I_{t}}{\partial z}\right|} .
$$

It is shown below that the noise in the current measurement $\delta I_{t}$ is small and that $\partial I_{t} / \partial z$ is quite large; consequently the vertical noise in an STM is very small.

The dominating noise sources in the tunneling current are the Johnson noise of the feedback resistor $R$ in the current amplifier, the Johnson noise in the tunneling junction, and the input noise of the operational amplifier. The Johnson noise density of a resistor $R$ at temperature $T$ is given by (Horowitz and Hill, 1989)

$$
n_{R}=\sqrt{4 k_{B} T R},
$$

where $k_{B}$ is the Boltzmann constant. In typical STM's, the tunneling current is of the order of $I_{t} \approx 100 \mathrm{pA}$ and is measured with an acquisition bandwidth of $B \approx 1 \mathrm{kHz}$, where $B$ is roughly determined by the spatial frequency of features that are to be scanned times the scanning speed. Thus, for a spatial frequency of 4 atoms $/ \mathrm{nm}$ and a scanning speed of $250 \mathrm{~nm} / \mathrm{s}$, a bandwidth of $B=1 \mathrm{kHz}$ is sufficient to map each atom as a single sinusoidal wave.

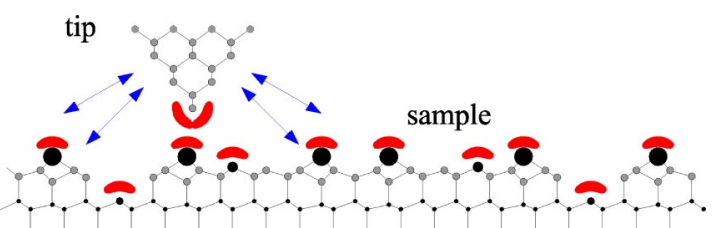

FIG. 6. (Color in online edition) Schematic view of an AFM tip close to a sample. Chemical short-range forces act when tip and sample orbitals (crescents) overlap. Long range forces (indicated with arrows) originate in the full volume and surface of the tip and are a critical function of the mesoscopic tip shape.

With a gain of $V / I=R=100 \mathrm{M} \Omega$ and $T=300 \mathrm{~K}$, the rms voltage noise is $n_{i} \sqrt{B}=\sqrt{4 k_{B} T R B}=40 \mu \mathrm{V}$ at room temperature, corresponding to a current noise of $\delta I_{t}$ $=0.4$ pA. With Eqs. (1) and (4), the vertical noise is

$$
\delta z_{I_{t}} \approx \frac{\sqrt{4 k_{B} T B / R}}{2 \kappa_{t}\left|I_{t}\right|}
$$

which amounts to a $z$ noise of $0.2 \mathrm{pm}$ in the present example. Thus in an STM the thermal noise in the tunneling current is not critical, because it is much smaller than the required resolution. It is interesting to note that the noise in an STM increases proportional to the square root of the required bandwidth $B$, a moderate rate compared to the $B^{1.5}$ dependence which holds for frequencymodulation atomic force microscopy [see Eq. (53)].

The spectacular spatial resolution and relative ease of obtaining atomic resolution by scanning tunneling microscopy rests on three properties of the tunneling current:

- As a consequence of the strong distance dependence of the tunneling current, even with a relatively blunt tip the chance is high that a single atom protrudes far enough out of the tip that it carries the main part of the tunneling current;

- Typical tunneling currents are in the nanoampere range-measuring currents of this magnitude can be done with a very good signal-to-noise ratio even with a simple experimental setup;

- Because the tunneling current is a monotonic function of the tip-sample distance, it is easy to establish a feedback loop that controls the distance so that the current is constant.

It is shown in the next section that none of these conditions is met in the case of the AFM, and therefore substantial hurdles had to be overcome before atomic resolution by AFM became possible.

\section{B. Tip-sample forces $F_{t s}$}

The AFM is similar to an STM, except that the tunneling tip is replaced by a force sensor. Figure 6 shows a sharp tip close to a sample. The potential energy between the tip and sample $V_{t s}$ causes a $z$ component of the tip-sample force $F_{t s}=-\partial V_{t s} / \partial z$ and a tip-sample 
spring constant $k_{t s}=-\partial F_{t s} / \partial z$. Depending on the mode of operation, the AFM uses $F_{t s}$ or some entity derived from $F_{t s}$ as the imaging signal.

Unlike the tunneling current, which has a very short range, $F_{t s}$ has long- and short-range contributions. We can classify the contributions by their range and strength. In vacuum, there are short-range chemical forces (fractions of $\mathrm{nm}$ ) and van der Waals, electrostatic, and magnetic forces with a long range (up to $100 \mathrm{~nm}$ ). In ambient conditions, meniscus forces formed by adhesion layers on tip and sample (water or hydrocarbons) can also be present.

A prototype of the chemical bond is treated in many textbooks on quantum mechanics (see, for example, Baym, 1969): the $\mathrm{H}_{2}{ }^{+}$ion is a model for the covalent bond. This quantum-mechanical problem can be solved analytically and gives interesting insights into the character of chemical bonds. The Morse potential (see, for example, Israelachvili, 1991)

$$
V_{\text {Morse }}=-E_{\text {bond }}\left(2 e^{-\kappa(z-\sigma)}-e^{-2 \kappa(z-\sigma)}\right)
$$

describes a chemical bond with bonding energy $E_{\text {bond }}$, equilibrium distance $\sigma$, and a decay length $\kappa$. With a proper choice of $E_{\text {bond }}, \sigma$, and $\kappa$, the Morse potential is an excellent fit for the exact solution of the $\mathrm{H}_{2}{ }^{+}$problem.

The Lennard-Jones potential (see, for example, Ashcroft and Mermin, 1981; Israelachvili, 1991),

$$
V_{\text {Lennard-Jones }}=-E_{\text {bond }}\left(2 \frac{z^{6}}{\sigma^{6}}-\frac{z^{12}}{\sigma^{12}}\right),
$$

has an attractive term $\propto r^{-6}$ originating from the van der Waals interaction (see below) and a repulsive term $\propto r^{-12}$.

While the Morse potential can be used for a qualitative description of chemical forces, it lacks an important property of chemical bonds: anisotropy. Chemical bonds, especially covalent bonds, show an inherent angular dependence of the bonding strength (see Pauling, 1957 and Coulson and McWeeny, 1991). Empirical models which take the directionality of covalent bonds into account are the Stillinger-Weber potential (Stillinger and Weber, 1985), the Tersoff potential, and others. For a review see Bazant and Kaxiras (1997) and references therein. The Stillinger-Weber (SW) potential appears to be a valid model for the interaction of silicon tips with silicon samples in AFM. As Bazant and Kaxiras (1997) write,

"Although the various terms [of the Stillinger-Weber potential] lose their physical significance for distortions of the diamond lattice large enough to destroy $s p^{3} h y$ bridization, the $S W$ potential seems to give a reasonable description of many states experimentally relevant, such as point defects, certain surface structures, and the liquid and amorphous states" (Bazant and Kaxiras, 1997).

Using the Stillinger-Weber potential, one can explain subatomic features in Si images (Giessibl, Hembacher, et al., 2000). Qualitatively, these findings have been reproduced with ab initio calculations (Huang et al., 2003). The Stillinger-Weber potential necessarily contains nearest- and next-nearest-neighbor interactions. Unlike solids with a face-centered-cubic or body-centered-cubic lattice structure, solids that crystallize in the diamond structure are unstable when only next-neighbor interactions are taken into account. The nearest-neighbor contribution of the Stillinger-Weber potential is

$$
\begin{aligned}
V_{n}(r)= & E_{\text {bond }} A\left[B\left(\frac{r}{\sigma^{\prime}}\right)^{-p}-\left(\frac{r}{\sigma^{\prime}}\right)^{-q}\right] \\
& \times e^{1 /\left(r / \sigma^{\prime}-a\right)} \text { for } r<a \sigma^{\prime} \text {, else } V_{n n}(r)=0 .
\end{aligned}
$$

The next-nearest-neighbor contribution is

$$
\begin{aligned}
V_{n n}\left(\mathbf{r}_{i}, \mathbf{r}_{j}, \mathbf{r}_{k}\right)= & E_{\text {bond }}\left[h\left(r_{i j}, r_{i k}, \theta_{j i k}\right)+h\left(r_{j i}, r_{j k}, \theta_{i j k}\right)\right. \\
& \left.+h\left(r_{k i}, r_{k j}, \theta_{i k j}\right)\right]
\end{aligned}
$$

with

$$
\begin{aligned}
h\left(r_{i j}, r_{i k}, \theta_{j i k}\right)= & \lambda e^{\gamma\left[1 /\left(r_{i j} / \sigma^{\prime}-a\right)+1 /\left(r_{i k} / \sigma^{\prime}-a\right)\right]} \\
& \times\left(\cos \theta_{j i k}+\frac{1}{3}\right)^{2} \\
& \text { for } r_{i j, i k}<a \sigma^{\prime}, \text { else } 0 .
\end{aligned}
$$

Stillinger and Weber found optimal agreement with experimental data for the following parameters:

$$
\begin{aligned}
& A=7.049556277, \quad p=4, \quad \gamma=1.20, \\
& B=0.6022245584, \quad q=0, \quad \lambda=21.0, \\
& E_{\text {bond }}=3.4723 \text { aJ, } \quad a=1.8, \quad \sigma^{\prime}=2.0951 \AA .
\end{aligned}
$$

The equilibrium distance $\sigma$ is related to $\sigma^{\prime}$ by $\sigma$ $=2^{1 / 6} \sigma^{\prime}$. The potential is constructed in such a way as to ensure that $V_{n}$ and $V_{n n}$ and all their derivatives with respect to distance vanish for $r>a \sigma^{\prime}=3.7718 \AA$. The diamond structure is favored by the Stillinger-Weber potential because of the factor $\left(\cos \theta+\frac{1}{3}\right)^{2}$-this factor is zero when $\theta$ equals the tetrahedron bond angle of $\theta$ $=109.47^{\circ}$.

With increasing computer power, it becomes more and more feasible to perform ab initio calculations for tip-sample forces. See, for example, Perez et al. (1997, 1998); Ke et al. (2001); Tobik et al. (2001); Huang et al. (2003).

The van der Waals interaction is caused by fluctuations in the electric dipole moment of atoms and their mutual polarization. For two atoms at distance $z$, the energy varies as $1 / z^{6}$ (Baym, 1969). Assuming additivity and disregarding the discrete nature of matter by replacing the sum over individual atoms by an integration over a volume with a fixed number density of atoms, the van der Waals interaction between macroscopic bodies can be calculated by the Hamaker approach (Hamaker, 1937). This approach does not account for retardation effects due to the finite speed of light and is therefore only appropriate for distances up to several hundred angstroms. For a spherical tip with radius $R$ next to a flat surface $(z$ is the distance between the plane connecting the centers of the surface atoms and the center of the closest tip atom) the van der Waals potential is given by (Israelachvili, 1991) 


$$
V_{v d W}=-\frac{A_{H} R}{6 z} .
$$

The van der Waals force for spherical tips is thus proportional to $1 / z^{2}$, while for pyramidal and conical tips, a $1 / z$ force law holds (Giessibl, 1997). The Hamaker constant $A_{H}$ depends on the type of materials (atomic polarizability and density) of the tip and sample. For most solids and interactions across a vacuum, $A_{H}$ is of the order of $1 \mathrm{eV}$. For a list of $A_{H}$ for various materials, see Krupp (1967) and French (2000). The van der Waals interaction can be quite large-the typical radius of an etched metal tip is $100 \mathrm{~nm}$ and with $z=0.5 \mathrm{~nm}$, the van der Waals energy is $\approx-30 \mathrm{eV}$, and the corresponding force is $\approx-10 \mathrm{nN}$. Because of their magnitude, van der Waals forces are a major disturbance in force microscopy. Ohnesorge and Binnig (1993) have shown (see Sec. IV) that large background van der Waals forces can be reduced dramatically by immersing the cantilever in water.

A more modern approach to the calculation of van der Waals forces is described by Hartmann (1991).

When the tip and sample are both conductive and have an electrostatic potential difference $U \neq 0$, electrostatic forces are important. For a spherical tip with radius $R$, the potential energy is given by Sarid (1994). If the distance between a flat surface and a spherical tip with radius $R$ is small compared to $R$, the force is approximately given by (see Olsson, Lin, Yakimov, and Erlandsson, 1998; Law and Rieutord, 2002)

$$
F_{\text {electrostatic }}(z)=-\frac{\pi \epsilon_{0} R U^{2}}{d} .
$$

Like the van der Waals interaction, the electrostatic interaction can also cause large forces-for a tip radius of $100 \mathrm{~nm}, U=1 \mathrm{~V}$, and $z=0.5 \mathrm{~nm}$, the electrostatic force is $\approx-5.5 \mathrm{nN}$.

It is interesting to note that short-range van der Waals forces (energy $\propto 1 / z^{6}$ ) add up to long-range overall tipsample forces because of their additivity. The opposite effect can occur with electrostatic forces: in ionic crystals, where adjacent atoms carry opposite charges, the envelope of the electrostatic field has a short-range exponential distance dependence (Giessibl, 1992). ${ }^{1}$

\section{The force sensor (cantilever)}

Tip-sample forces can vary strongly on the atomic scale, and Pethica (1986) has proposed that they even explain artifacts like giant corrugations apparent in STM experiments. However, it is difficult to isolate force effects in scanning tunneling microscopy, and a dedicated

\footnotetext{
${ }^{1}$ More information about tip-sample forces can be found in Ciraci et al. (1990); Israelachvili (1991); Sarid (1994); Perez et al. (1997, 1998); Shluger et al. (1997, 1999); Abdurixit et al. (1999); Drakova (2001); Ke et al. (2001, 2002); Tobik et al. (2001); Foster et al. (2002); Garcia and Perez (2002); Tsukada et al. (2002) and references therein.
}

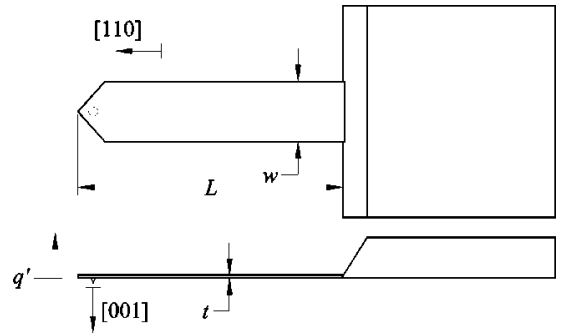

FIG. 7. Top view and side view of a microfabricated cantilever (schematic). Most cantilevers have this diving-board geometry.

sensor for detecting forces is needed. The central element of a force microscope and its major instrumental difference from a scanning tunneling microscope is the spring which senses the force between tip and sample. For sensing normal tip-sample forces, the force sensor should be rigid in two axes and relatively soft in the third axis. This property is fulfilled with a cantilever beam, and therefore the cantilever geometry is typically used for force detectors. A generic cantilever is shown in Fig. 7. For a rectangular cantilever with dimensions $w, t$, and $L$ (see Fig. 7), the spring constant $k$ is given by (Chen, 1993)

$$
k=\frac{Y w t^{3}}{4 L^{3}}
$$

where $Y$ is Young's modulus. The fundamental eigenfrequency $f_{0}$ is given by (Chen, 1993)

$$
f_{0}=0.162 \frac{t}{L^{2}} \sqrt{\frac{Y}{\rho}},
$$

where $\rho$ is the mass density of the cantilever material.

The properties of interest are the stiffness $k$, the eigenfrequency $f_{0}$, the quality factor $Q$, the variation of the eigenfrequency with temperature $\partial f_{0} / \partial T$, and of course the chemical and structural composition of the tip. The first AFM's were mostly operated in the static contact mode (see below), and for this mode the stiffness of the cantilever should be less than the interatomic spring constants of atoms in a solid (Rugar and Hansma, 1990), which amounts to $k \leqslant 10 \mathrm{~N} / \mathrm{m}$. This constraint on $k$ was assumed to hold for dynamic atomic force microscopy, as well. However, it turned out later that in dynamic atomic force microscopy, $k$ values exceeding hundreds of $\mathrm{N} / \mathrm{m}$ help to reduce noise and increase stability (Giessibl, Bielefeldt, et al., 1999). The $Q$ factor depends on the damping mechanisms present in the cantilever. For micromachined cantilevers operated in air, $Q$ is mainly limited by viscous drag and typically amounts to a few hundred, while in vacuum, internal and surface effects in the cantilever material are responsible for damping and $Q$ reaches hundreds of thousands.

The first cantilevers were made from a gold foil with a small diamond tip attached to it (Binnig, 1986). Simple cantilevers can even be cut from household aluminum foil (Rugar and Hansma, 1990) and etched tungsten wires (McClelland et al., 1987). Later, silicon micromachining technology was employed to build cantilevers in 


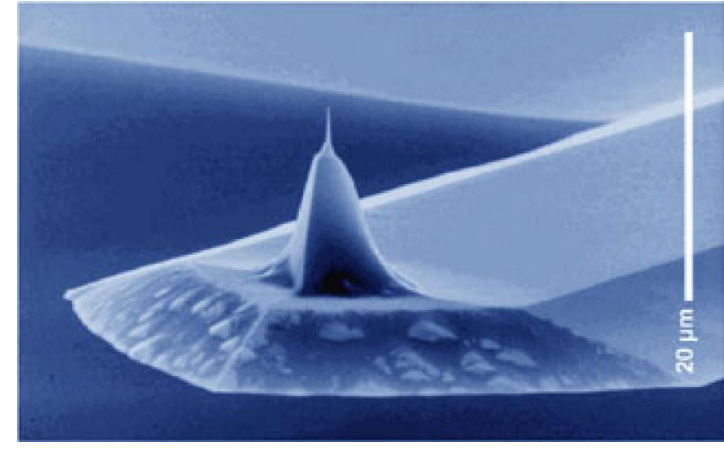

FIG. 8. (Color in online edition) Scanning electron micrograph of a micromachined silicon cantilever with an integrated tip pointing in the [001] crystal direction (Wolter et al., 1991). This is a Pointprobe sensor made by Nanosensors $\mathrm{GmbH}$ und Co. KG, Norderfriedrichskoog, Germany D-25870. Photo courtesy of Nanosensors GmbH \& Co. KG.

parallel production with well-defined mechanical properties. The first micromachined cantilevers were built at Stanford in the group of Calvin F. Quate. Initially, massproduced cantilevers were built from $\mathrm{SiO}_{2}$ and $\mathrm{Si}_{3} \mathrm{~N}_{4}$ (Albrecht et al., 1990). Later, cantilevers with integrated tips were machined from silicon-on-insulator wafers (Akamine et al., 1990). The most common cantilevers in use today are built from all-silicon with integrated tips pointing in a [001] crystal direction; these were developed by Wolter, Bayer, and Greschner (1991) at IBM Sindelfingen, Germany. Figures 8 and 9 show the type of cantilevers that are mainly used today: micromachined silicon cantilevers with integrated tips. Tortonese, Barrett, and Quate (1993) have built self-sensing cantilevers with integrated tips and a built-in deflection-measuring scheme utilizing the piezoresistive effect in silicon (see Fig. 10).

In dynamic atomic force microscopy, some requirements for the force sensor are similar to the desired properties of the time-keeping element in a watch: ut-

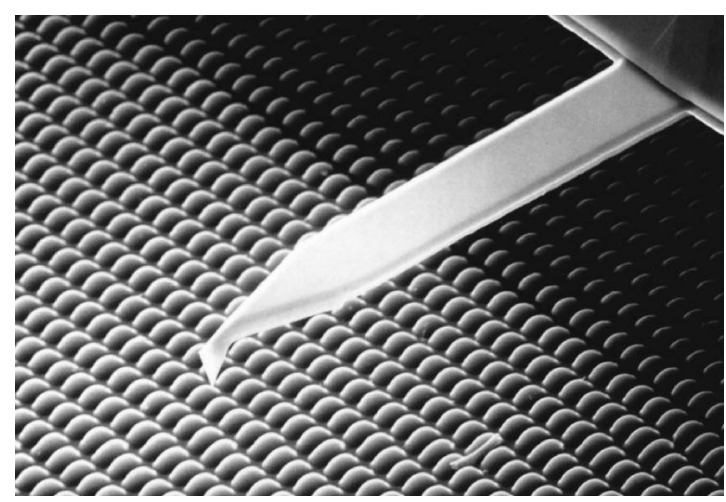

FIG. 9. Scanning electron micrograph of a micromachined silicon cantilever with an integrated tip pointing in the [001] crystal direction. In this type, the tip is etching free so that the sample area adjacent to the tip is visible in an optical microscope. Length, $120 \mu \mathrm{m}$; width, $30 \mu \mathrm{m}$; thickness, $2.8 \mu \mathrm{m} ; k$ $=15 \mathrm{~N} / \mathrm{m} ; f_{0}=300 \mathrm{kHz}$. Photo courtesy of Olympus Optical Co. Ltd, Hachioji, Tokyo 192-8507, Japan.

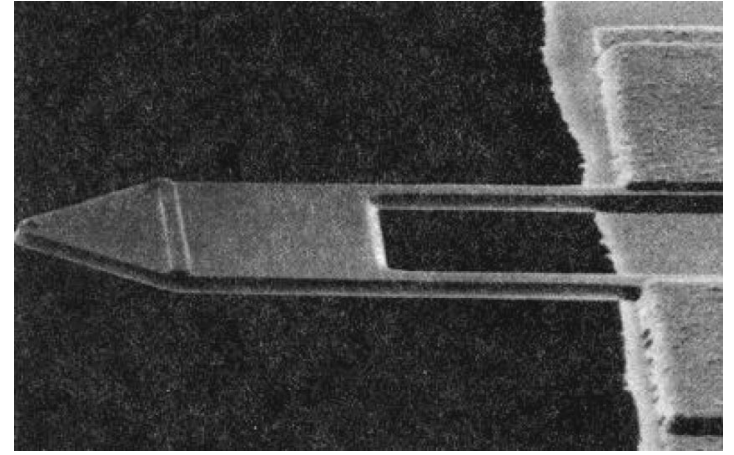

FIG. 10. Scanning electron micrograph of a piezoresistive cantilever built from silicon. Length, $250 \mu \mathrm{m}$; full width, $80 \mu \mathrm{m}$; thickness, $2 \mu \mathrm{m}$. From Tortonese et al., 1993.

most frequency stability over time and temperature changes and little energy consumption. Around 1970, the watch industry was revolutionized with the introduction of quartz tuning forks as frequency standards in clocks (Walls, 1985; Momosaki, 1997). Billions of these devices are now manufactured annually, and the deviations of even low-cost watches are no more than a few seconds a week. Experimental studies of using quartzbased force sensors were carried out soon after the invention of the AFM. Güthner et al. (1989) and Güthner (1992) used tuning forks as force sensors in acoustic near-field microscopy, while Karrai and Grober (1995) used a tuning fork to control the distance between the optical near-field probe and the surface in a scanning near-field-optical microscope. Bartzke et al. (1993) proposed the "needle sensor," a force sensor based on a quartz bar oscillator. Rychen et al. (1999) and Hembacher et al. (2002) demonstrated the use of quartz tuning forks at low temperature, and other applications of quartz tuning forks as force sensors can be found in Edwards et al. (1997); Ruiter et al. (1997); Todorovic and Schulz (1998); Tsai and Lu (1998); Wang (1998); and Rensen et al. (1999). Quartz tuning forks have many attractive properties, but their geometry gives them marked disadvantages for use as force sensors. The great benefit of the fork geometry is the high $Q$ factor, which is a consequence of the presence of an oscillation mode in which both prongs oscillate opposite to each other. The dynamic forces necessary to keep the two prongs oscillating cancel in this case exactly. However, this only works if the eigenfrequency of both prongs matches precisely. The mass of the tip mounted on one prong and the interaction of this tip with a sample breaks the symmetry of tuning fork geometry. This problem can be avoided by fixing one of the two beams and turning the fork symmetry into a cantilever symmetry, where the cantilever is attached to a high-mass substrate with a low-loss material. Figure 11 shows a quartz cantilever based on a quartz tuning fork (Giessibl, 1996, 1998, 2000). Quartz tuning forks are available in several sizes. We have found optimal performance with the type of tuning fork used in Swatch wristwatches. In contrast to micromachined silicon cantilevers, the quartz forks are large. Therefore a wide selection of tips can be mounted 


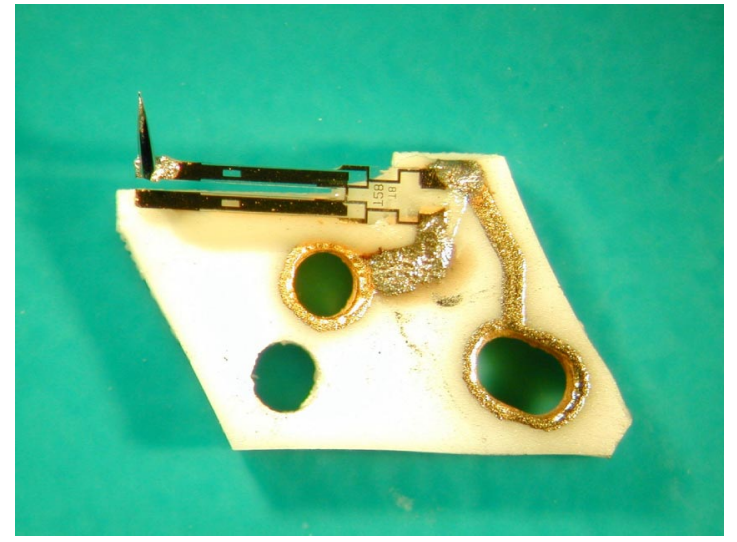

FIG. 11. (Color in online edition) Micrograph of a "qPlus" sensor-a cantilever made from a quartz tuning fork. One of the prongs is fixed to a large substrate and a tip is mounted to the free prong. Because the fixed prong is attached to a heavy mass, the device is mechanically equivalent to a traditional cantilever. The dimensions of the free prong: length, $2400 \mu \mathrm{m}$; width, $130 \mu \mathrm{m}$; thickness, $214 \mu \mathrm{m}$.

on a tuning fork with the mere help of tweezers and a stereoscopic microscope-sophisticated micromachining equipment is not needed. Tips made from tungsten, diamond, silicon, iron, cobalt, samarium, CoSm permanent magnets, and iridium have been built in our laboratory for various purposes. Figure 12 shows a quartz cantilever oriented for lateral force detection (see Sec. X.D; Giessibl, Herz, and Mannhart, 2002). Piezoelectric sensors based on thin films of materials with much higher piezoelectric constants than quartz (Itoh et al., 1996) are also available. However, these devices lack the very low internal dissipation and high-frequency stability of quartz. The general advantage of piezoelectric sensors versus piezoresistive sensors is that the latter dissipate power in the $\mathrm{mW}$ range, while electric dissipation is negligible in piezoelectric sensors. Therefore piezoelectric sensors are preferred over piezoresistive schemes for low-temperature applications.

\section{Cantilever tips}

For atomic-resolution atomic force microscopy, the front atom of the tip should ideally be the only atom that interacts strongly with the sample. In order to reduce the forces caused by the shaft of the tip, the tip radius should be as small as possible (see Sec. II.B). Cantilevers made of silicon with integrated tips are typically oriented so that the tip points in the [001] crystal direction. Due to the anisotropic etching rates of $\mathrm{Si}$ and $\mathrm{SiO}_{2}$, these tips can be etched so that they develop a very sharp apex (Marcus et al., 1990), as shown in Fig. 13. Recently, it has turned out that not only the sharpness of a tip is important for atomic force microscopy, but also the coordination of the front atom. Tip and sample can be viewed as two giant molecules (Chen, 1993). In chemical reactions between two atoms or molecules, the chemical identity and the spatial arrangement of both partners plays a crucial role. For atomic

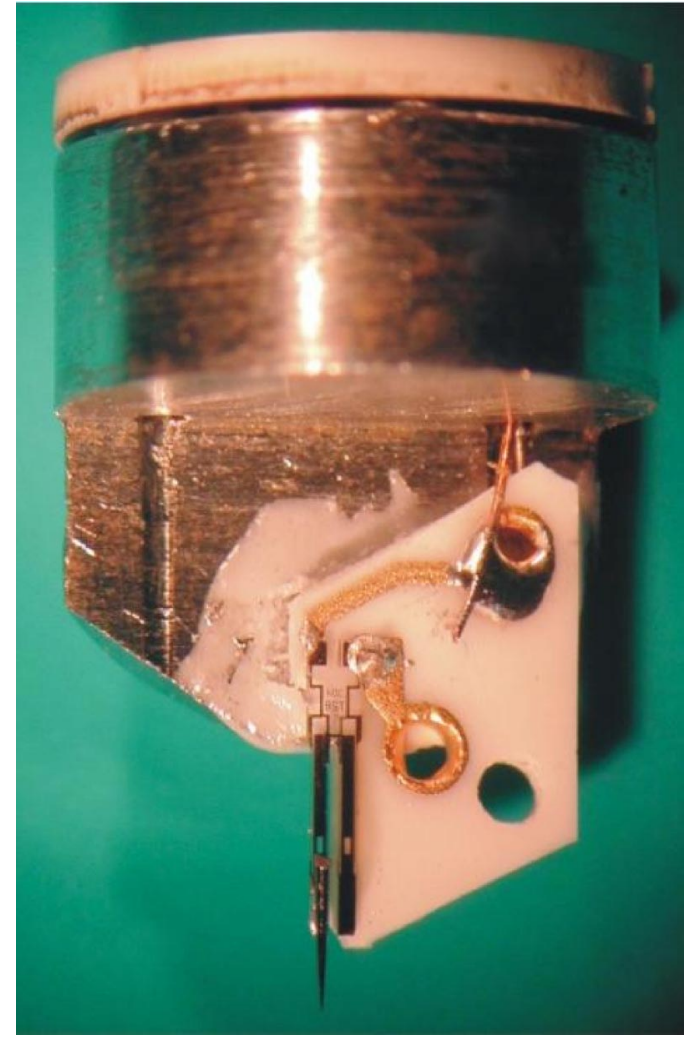

FIG. 12. (Color in online edition) Micrograph of a "qPlus" lateral force sensor. The lateral force sensor is similar to the normal force sensor in Fig. 11. It is rotated $90^{\circ}$ with respect to the normal force sensor and its tip is aligned parallel to the free prong.

force microscopy with true atomic resolution, the chemical identity and bonding configuration of the front atom is therefore critical. In [001]-oriented silicon tips, the front atom exposes two dangling bonds (if bulk termination is assumed) and has only two connecting bonds to the rest of the tip. If we assume bulk termination, it is immediately evident that tips pointing in the [111] direction are more stable, because then the front atom has three bonds to the rest of the tip (see Figs. 14). In a simple picture where only nearest-neighbor interactions are contributing significantly to the bonding energy, the front atom of a [111]-oriented silicon tip has 3/4 of the bulk atomic bonding energy. For a [111]-oriented metal tip with fcc bulk structure, the bonding energy of the front atom has only $3 / 12$ of the bulk value. This trivial picture might explain why silicon can be imaged with atomic resolution using positive frequency shifts (i.e., repulsive forces) with a [111] silicon tip (to be discussed below). Even if the [111] sidewalls of these tips reconstruct to, say, Si $7 \times 7$, the front atom is fixed by three bonds, and a very stable tip should emerge. Figure 15 shows a tip with [111] orientation. The tip is cleaved from a silicon wafer. Experiments show that these tips can come very close to a surface without getting damaged (Giessibl, Hembacher, et al., 2001b).

\section{Measurement of cantilever deflection and noise}

In the first AFM, the deflection of the cantilever was measured with an STM. The backside of the cantilever 


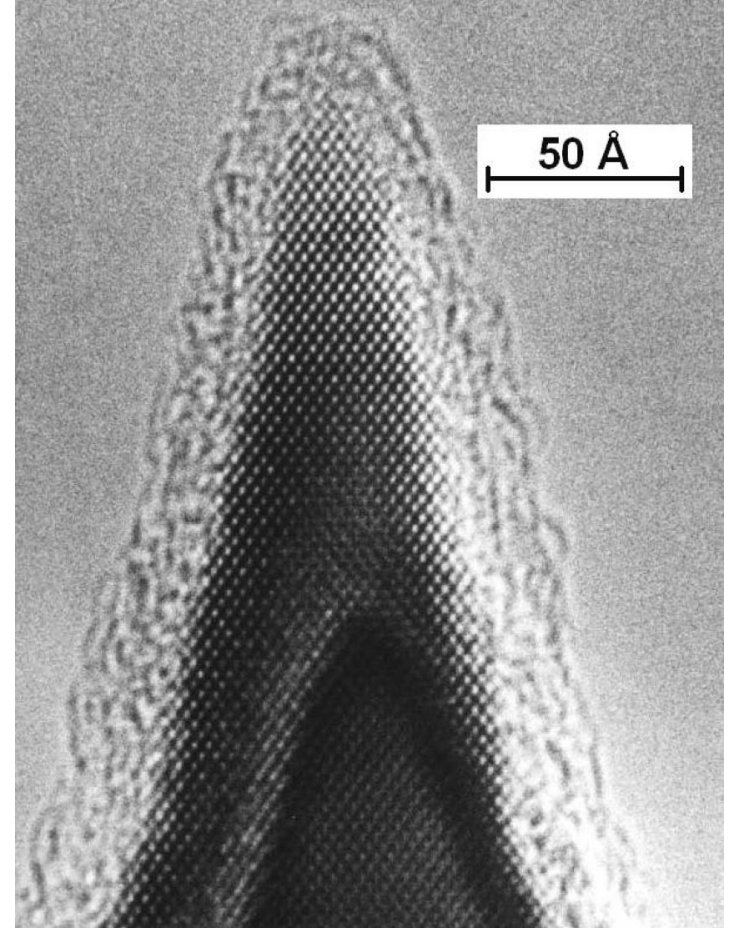

FIG. 13. Transmission electron micrograph of an extremely sharp silicon tip. The native oxide has been etched away with hydrofluoric acid before imaging. The 15-20- $\AA$-thick coating of the tip is mostly due to hydrocarbons which have been polymerized by the electron beam. Interestingly, the crystal structure appears to remain bulklike up to the apex of the tip. From Marcus et al., 1990.

was metalized, and a tunneling tip was brought close to it to measure the deflection (Binnig et al., 1986). While the tunneling effect is very sensitive to distance variations, this method has a number of drawbacks:

- It is difficult to position a tunneling tip so that it aligns with the very small area at the end of the cantilever.

- The tunneling tip exerts forces on the cantilever, and it is impossible to distinguish between forces caused by cantilever-sample and cantilever-tunneling tip interactions.

- When the cantilever is deflected, the lateral position of the tip on the backside of the cantilever is shifted. (a)

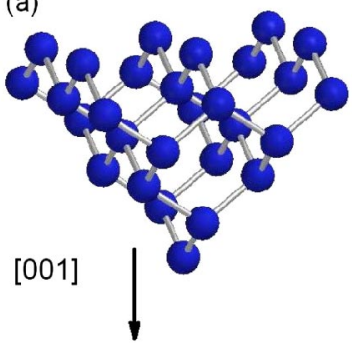

(b)

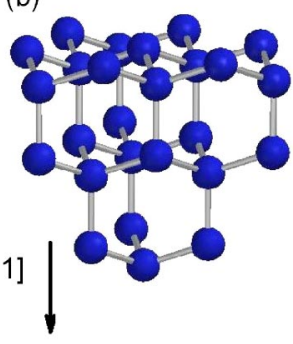

FIG. 14. (Color in online edition) Model of atomic arrangements for bulklike terminated silicon tips, (a) pointing in a [001] direction and (b) in a [111] direction.

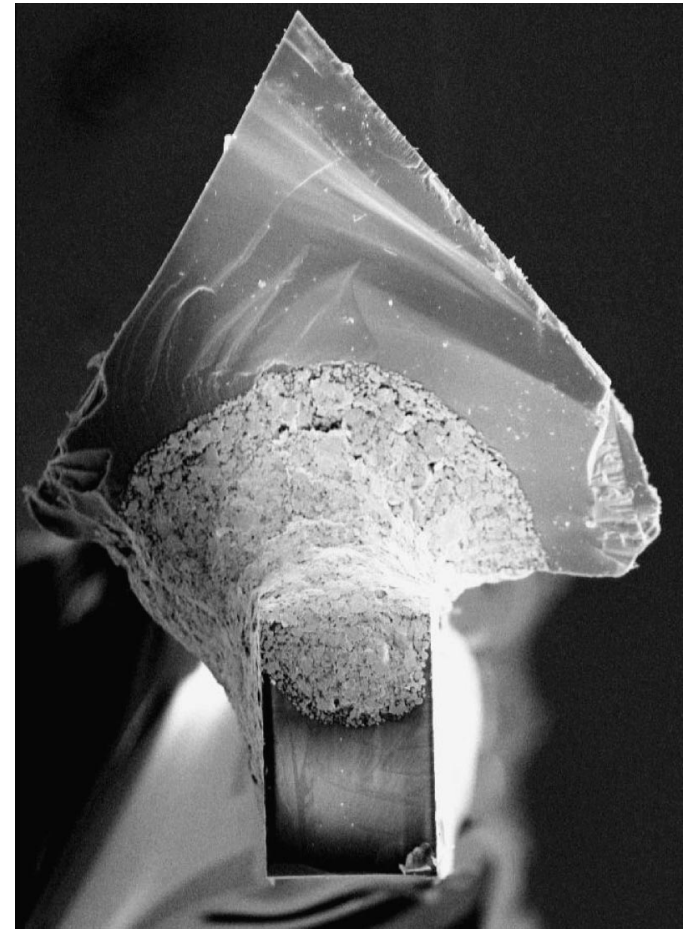

FIG. 15. Scanning electron micrograph of a cleaved singlecrystal silicon tip attached to the free prong of a qPlus sensor. The rectangular section is the end of the free prong with a width of $130 \mu \mathrm{m}$ and a thickness of $214 \mu \mathrm{m}$. The tip is pointed in the [111] direction and bounded by ( $\overline{1} \overline{1} \overline{1}),(1 \overline{1} \overline{1})$, and (111) planes according to the method of Giessibl et al. (2001b). Figure courtesy of Christian Schiller taken from Schiller, 2003.

The atomic roughness of the cantilever backside along with the lateral motion results in a nonlinear deflection signal.

Subsequent designs used optical (interferometer, beambounce) or electrical methods (piezoresistive, piezoelectric) for measuring the cantilever deflection. The deflection of silicon cantilevers is most commonly measured by optical detection through an interferometer or by bouncing a light beam off the cantilever and measuring its deflection (the "beam bounce method"). For detailed descriptions of these techniques, see Sarid (1994); optical detection techniques are discussed extensively by Howald (1994). The deflection of piezoresistive cantilevers is usually measured by making them part of a Wheatstone bridge (see Tortonese et al., 1993).

The deflection of the cantilever is subject to thermal drift and other noise factors. This can be expressed in a plot of the deflection noise density versus frequency. A typical noise density is plotted in Fig. 16, showing a $1 / f$ dependence for low frequency that merges into a constant noise density ("white noise") above the " $1 / f$ corner frequency." This $1 / f$ noise is also apparent in macroscopic force-sensing devices, such as scales. Typically, scales have a reset or zero button, which allows the user to reset the effects of long-term drift. Machining AFM's from materials with low thermal expansion coefficients 


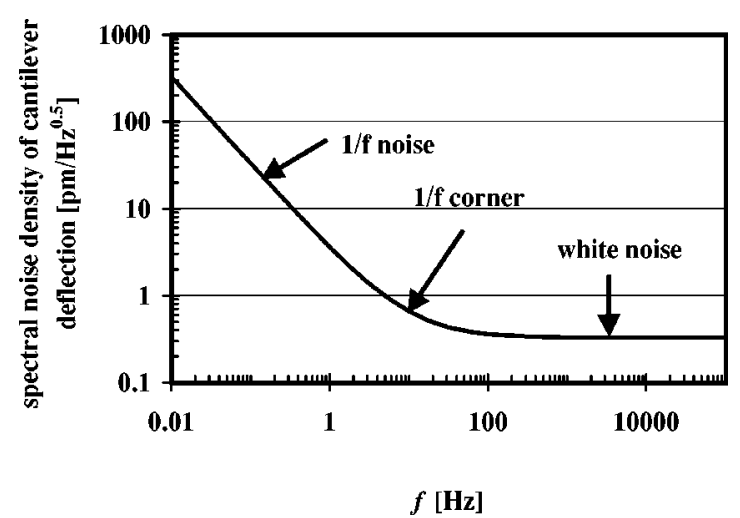

FIG. 16. Noise spectrum of a typical cantilever deflection detector (schematic), characterized by $1 / f$ noise for low frequencies and white noise for intermediate frequencies. For very high frequencies, the deflection noise density of typical cantilever deflection sensors goes up again ("blue noise," not shown here).

like Invar or operation at low temperatures helps to minimize $1 / f$ noise.

\section{Thermal stability}

A change in temperature can cause bending of the cantilever and a change in its eigenfrequency. In this respect, quartz is clearly superior to silicon as a cantilever material, as quartz can be cut along specific crystal orientations such that the variation of oscillation frequency of a tuning fork or cantilever is zero for a certain temperature $T_{0}$. For quartz cut in the $X+5^{\circ}$ direction, $T_{0}$ $\approx 300 \mathrm{~K}$, see, for example, Momosaki (1997). This cannot be accomplished with silicon cantilevers. In the dynamic operating modes (see Sec. VI), drifts in $f_{0}$, caused by variations in temperature, add to the vertical noise. The eigenfrequency [see Eq. (27)] is determined by the spring constant and the effective mass of the cantilever. The spring constant changes with temperature, due to thermal expansion and the change of Young's modulus $Y$ with temperature. Changes in the effective mass due to picking up a few atoms from the sample or transferring some atoms from the tip to the sample are insignificant, because a typical cantilever contains at least $10^{14}$ atoms. The resonance frequency of a cantilever is given in Eq. (15). With the velocity of sound in the cantilever material $v_{s}=\sqrt{Y / \rho}$, Eq. (15) can be expressed as (Chen, 1993)

$$
f_{0}=0.162 v_{s} \frac{t}{L^{2}} .
$$

The temperature dependence of the eigenfrequency is then given by

$$
\frac{1}{f_{0}} \frac{\partial f_{0}}{\partial T}=\frac{1}{v_{s}} \frac{\partial v_{s}}{\partial T}-\alpha
$$

where $\alpha$ is the thermal expansion coefficient. For silicon oriented along the [110] crystal direction (see Fig. 7), $\left(1 / v_{s}\right)\left(\partial v_{s} / \partial T\right)=-5.5 \times 10^{-5} \mathrm{~K}^{-1}$ and $\alpha=2.55 \times 10^{-6}$ $\mathrm{K}^{-1}$ at $T=290 \mathrm{~K}$ (Kuchling, 1982; Madelung, 1982). The resulting relative frequency shift for (rectangular) silicon cantilevers is then $-5.8 \times 10^{-5} \mathrm{~K}^{-1}$. This is a large noise source in classical FM-AFM, where relative frequency shifts can be as small as $-6 \mathrm{~Hz} / 151 \mathrm{kHz}=-4 \times 10^{-5}$ (see row 5 in Table I) and a temperature variation of $\Delta T=+0.69 \mathrm{~K}$ causes an equal shift in resonance frequency. The drift of $f_{0}$ with temperature is much smaller for cantilevers made of quartz. Figure 17 shows a comparison of typical frequency variations as a function of temperature for silicon and quartz. The data for silicon are calculated with Eq. (17), the quartz data are taken from Momosaki (1997). As can be seen, quartz is remarkably stable at room temperature compared to silicon. Less significant noise sources, like the thermal fluctuation of $A$, are discussed by Giessibl, Bielefeldt, et al. (1999). Hembacher et al. (2002) have measured the frequency variations of a quartz tuning fork sensor from room temperature to $5 \mathrm{~K}$.

\section{Operating modes of AFM's}

\section{Static atomic force microscopy}

In AFM, the force $F_{t s}$ which acts between the tip and sample is used as the imaging signal. In the static mode of operation, the force translates into a deflection $q^{\prime}$ $=F_{t s} / k$ of the cantilever. Because the deflection of the cantilever should be significantly larger than the deformation of the tip and sample, restrictions on the useful range of $k$ apply. In the static mode, the cantilever should be much softer than the bonds between the bulk atoms in tip and sample. Interatomic force constants in solids are in a range from $10 \mathrm{~N} / \mathrm{m}$ to about $100 \mathrm{~N} / \mathrm{m}$-in biological samples, they can be as small as $0.1 \mathrm{~N} / \mathrm{m}$. Thus typical values for $k$ in the static mode are $0.01-5 \mathrm{~N} / \mathrm{m}$. The eigenfrequency $f_{0}$ should be significantly higher than the desired detection bandwidth, i.e., if ten lines per second are recorded during imaging a width of say 100 atoms, $f_{0}$ should be at least $10 \times 2 \times 100 \mathrm{~s}^{-1}=2 \mathrm{kHz}$ in order to prevent resonant excitation of the cantilever.

Even though it has been demonstrated that atomic resolution is possible with static atomic force microscopy (Giessibl and Binnig, 1992b; Ohnesorge and Binng, 1993; Schimmel et al., 1999), the method can only be applied in certain cases. The magnitude of $1 / f$ noise can be reduced by low-temperature operation (Giessibl, 1992), where the coefficients of thermal expansion are very small, or by building the AFM of a material with a low thermal expansion coefficient. The long-range attractive forces have to be canceled by immersing tip and sample in a liquid (Ohnesorge and Binnig, 1993) or by partly compensating for the attractive force by pulling at the cantilever after jump-to-contact has occurred (Giessibl, 1991, 1992; Giessibl and Binnig, 1992b). Jarvis et al. $(1997,1996)$ have introduced a method for cancelling the long-range attractive force with an electromagnetic force applied to the cantilever.

While the experimental realization of static atomic force microscopy is difficult, the physical interpretation 
TABLE I. Operating parameters of various FM-AFM experiments: *, early experiments with nearly atomic resolution, experiments with standard parameters (classic NC-AFM) on semiconductors, metals, and insulators; **, small-amplitude experiments; ***, internal cantilever damping calculated from $\Delta E=2 \pi E / Q$. When $Q$ is not quoted in the original publication, a $Q$ value of 50000 is used as an estimate.

\begin{tabular}{|c|c|c|c|c|c|c|c|c|c|c|}
\hline Year & $\begin{array}{c}k \\
\mathrm{~N} / \mathrm{m}\end{array}$ & $\begin{array}{c}f_{0} \\
\mathrm{kHz}\end{array}$ & $\begin{array}{l}\Delta f \\
\mathrm{~Hz}\end{array}$ & $\begin{array}{c}A \\
\mathrm{~nm}\end{array}$ & $\begin{array}{c}\gamma \\
\mathrm{fN} \sqrt{\mathrm{m}}\end{array}$ & $\begin{array}{l}k A \\
\mathrm{nN}\end{array}$ & $\begin{array}{c}E \\
\mathrm{keV}\end{array}$ & $\begin{array}{l}\Delta E_{C L} \\
\mathrm{eV}^{* * *}\end{array}$ & Sample & Ref. \\
\hline $1994 *$ & 2.5 & 60.0 & -16 & 15.0 & -1.26 & 37.5 & 1.8 & 0.06 & $\mathrm{KCl}(001)$ & Giessibl and Trafas (1994) \\
\hline $1994 *$ & 2.5 & 60.0 & -32 & 3.3 & -0.29 & 8.25 & 0.1 & 0.4 & $\operatorname{Si}(111)$ & Giessibl (1994) \\
\hline 1995 & 17.0 & 114.0 & -70 & 34.0 & -66.3 & 544 & 61 & 14 & $\operatorname{Si}(111)$ & Giessibl (1995) \\
\hline 1995 & 43.0 & 276.0 & -60 & 40.0 & -75.6 & 1720 & 215 & 27 & $\operatorname{Si}(111)$ & Kitamura and Iwatsuki (1995) \\
\hline 1995 & 34.0 & 151.0 & -6 & 20.0 & -3.91 & 680 & 42 & 5 & $\operatorname{InP}(110)$ & Sugawara et al. (1995) \\
\hline 1996 & 23.5 & 153.0 & -70 & 19.0 & -28.8 & 447 & 27 & 3.3 & $\operatorname{Si}(111)$ & Lüthi et al. (1996) \\
\hline 1996 & 33.0 & 264.0 & -670 & 4.0 & -23.6 & 132 & 12 & 1.45 & $\operatorname{Si}(001)$ & Kitamura and Iwatsuki (1996) \\
\hline 1996 & 10.0 & 290.0 & -95 & 10.0 & -3.42 & 100 & 3.1 & 0.4 & $\operatorname{Si}(111)$ & Güthner (1996) \\
\hline 1997 & 30.0 & 168.0 & -80 & 13.0 & -21.9 & 390 & 16 & 2 & $\mathrm{NaCl}(001)$ & Bammerlin et al. (1997) \\
\hline 1997 & 28.0 & 270.0 & -80 & 15.0 & -15.7 & 420 & 20 & 2.5 & $\mathrm{TiO}_{2}(110)$ & Fukui et al. (1997) \\
\hline 1997 & 41.0 & 172.0 & -10 & 16.0 & -4.96 & 654 & 33 & 4 & $\operatorname{Si}(111)$ & Sugawara et al. (1997) \\
\hline 1999 & 35.0 & 160.0 & -63 & 8.8 & -10.1 & 338 & 10 & 1.4 & HOPG(0001) & Allers et al. (1999a) \\
\hline 1999 & 36.0 & 160.0 & -60.5 & 12.7 & -18.1 & 457 & 18 & 2.3 & InAs(110) & Schwarz et al. (1999) \\
\hline 1999 & 36.0 & 160.0 & -92 & 9.4 & -19.8 & 338 & 10 & 1.2 & $\mathrm{Xe}(111)$ & Allers et al. (1999b) \\
\hline 1999 & 27.4 & 152.3 & -10 & 11.4 & -2.2 & 312 & 11 & 1.4 & $\operatorname{Ag}(111)$ & Minobe et al. (1999) \\
\hline 2000 & 28.6 & 155.7 & -31 & 5.0 & -4.1 & 143 & 2.2 & 0.04 & $\operatorname{Si}(111)$ & Lantz et al. (2000) \\
\hline 2000 & 30.0 & 168.0 & -70 & 6.5 & -6.6 & 195 & 4.0 & 0.5 & $\mathrm{Cu}(111)$ & Loppacher et al. (2000) \\
\hline 2001 & 3.0 & 75.0 & -56 & 76 & -46.9 & 228 & 54.1 & 7 & $\mathrm{Al}_{2} \mathrm{O}_{3}(0001)$ & Barth and Reichling (2001) \\
\hline 2002 & 24.0 & 164.7 & -8 & 12.0 & -1.5 & 288 & 2.2 & 1.4 & $\mathrm{KCl}_{0.6} \mathrm{Br}_{0.4}$ & Bennewitz, Pfeiffer, et al. (2002) \\
\hline 2002 & 46.0 & 298.0 & -20 & 2.8 & -0.46 & 129 & 1.1 & 0.13 & $\operatorname{Si}(111)$ & Eguchi and Hasegawa (2002) \\
\hline $2000 * *$ & 1800 & 16.86 & -160 & 0.8 & -387 & 1440 & 3.6 & 11 & $\operatorname{Si}(111)$ & Giessibl et al. (2000) \\
\hline $2001 * *$ & 1800 & 20.53 & 85 & 0.25 & +29.5 & 450 & 0.4 & 1 & $\operatorname{Si}(111)$ & Giessibl, Bielefeldt, et al. (2001) \\
\hline
\end{tabular}

of static AFM images is simple: The image is a map $z\left(x, y, F_{t s}=\right.$ const $)$.

\section{Dynamic atomic force microscopy}

In the dynamic operation modes, the cantilever is deliberately vibrated. The cantilever is mounted on an actuator to allow the external excitation of an oscillation. There are two basic methods of dynamic operation: amplitude-modulation (AM) and frequency-modulation

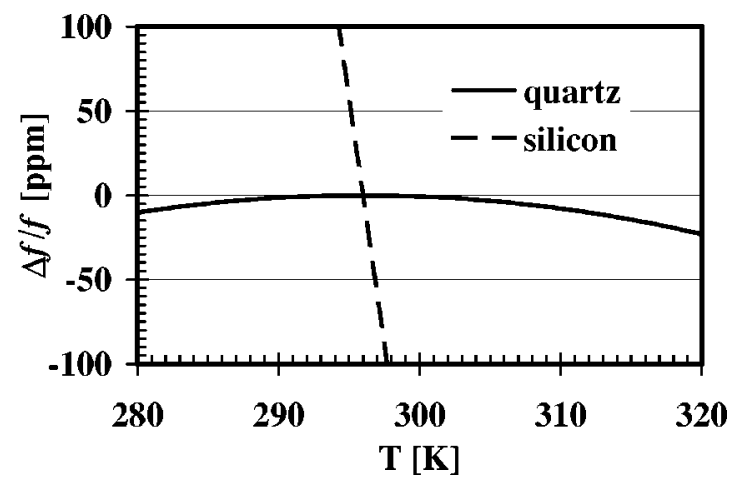

FIG. 17. Frequency variation as a function of temperature for silicon [110]-oriented cantilevers and quartz tuning forks in $\mathrm{X}$ $+5^{\circ}$ cut (see Momosaki, 1997).
(FM) operation. In AM-AFM (Martin, Williams, and Wickramasinghe, 1987), the actuator is driven by a fixed amplitude $A_{\text {drive }}$ at a fixed frequency $f_{\text {drive }}$, where $f_{\text {drive }}$ is close to but different from $f_{0}$. When the tip approaches the sample, elastic and inelastic interactions cause a change in both the amplitude and the phase (relative to the driving signal) of the cantilever. These changes are used as the feedback signal. The change in amplitude in AM mode does not occur instantaneously with a change in the tip-sample interaction, but on a time scale of $\tau_{A M} \approx 2 Q / f_{0}$. With $Q$ factors reaching 100000 in vacuum, the AM mode is very slow. Albrecht, Grutter, Horne, and Rugar (1991) solved this problem by introducing the frequency-modulation (FM) mode, in which the change in the eigenfrequency occurs within a single oscillation cycle on a time scale of $\tau_{F M} \approx 1 / f_{0}$.

Both AM and FM modes were initially meant to be "noncontact" modes, i.e., the cantilever was far away from the surface and the net force between the front atom of the tip and the sample was clearly attractive. The AM mode was later used very successfully at a closer distance range in ambient conditions involving repulsive tip-sample interactions ("Tapping Mode"; Zhong et al., 1993), and Erlandsson et al. (1997) obtained atomic resolution on $\mathrm{Si}$ in vacuum with an etched tungsten cantilever operated in AM mode in 1996. Using the FM mode in vacuum improved the resolution dramati- 


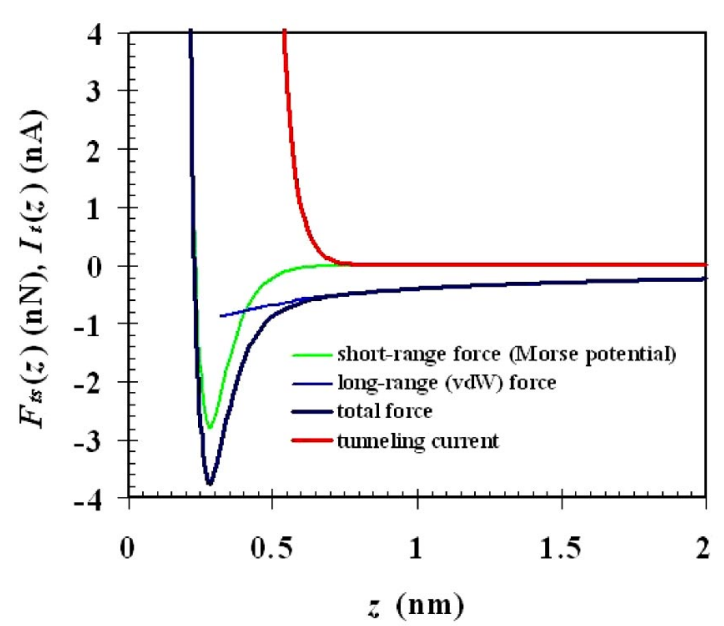

FIG. 18. (Color in online edition) Plot of tunneling current $I_{t}$ and force $F_{t s}$ (typical values) as a function of distance $z$ between center of front atom and plane defined by the centers of surface atom layer.

cally (Giessibl, 1994; Giessibl and Trafas, 1994). Finally atomic resolution (Giessibl, 1995) was obtained. A detailed description of the FM mode is given in Sec. VI.

\section{CHALLENGES FACED BY ATOMIC FORCE MICROSCOPY WITH RESPECT TO SCANNING TUNNELING MICROSCOPY}

In a scanning tunneling microscope, a tip has to be scanned across a surface with a precision of picometers while a feedback mechanism adjusts the $z$ position such that the tunneling current is constant. This task seems daunting, and the successful realization of scanning tunneling microscopy is an amazing accomplishment. Yet, implementing an AFM capable of atomic resolution poses even more obstacles. Some of these challenges become apparent when comparing the characteristics of the physical observables used in the two types of microscopes. Figure 18 is a plot of tunneling current and tipsample force as a function of distance. For experimental measurements of force and tunneling current, see, for example, Schirmeisen et al. (2000). The tunneling current is a monotonic function of the tip-sample distance and increases sharply with decreasing distance. In contrast, the tip-sample force has long- and short-range components and is not monotonic.

\section{A. Stability}

van der Waals forces in vacuum are always attractive, and if chemical bonding between tip and sample can occur, the chemical forces are also attractive for distances greater than the equilibrium distance. Because the tip is mounted on a spring, approaching the tip can cause a sudden jump-to-contact when the stiffness of the cantilever is less than a certain value.

This instability occurs in the quasistatic mode if

$$
k<\max \left(-\frac{\partial^{2} V_{t s}}{\partial z^{2}}\right)=k_{t s}^{\max }
$$

(Tabor and Winterton, 1969; McClelland et al., 1987; Burnham and Colton, 1989). The jump-to-contact can be avoided even for soft cantilevers by oscillating the cantilever at a large enough amplitude $A$ :

$$
k A>\max \left(-F_{t s}\right)=F_{t s}^{\max }
$$

(Giessibl, 1997). If hysteresis occurs in the $F_{t s}(z)$ relation, the energy $\Delta E_{t s}$ needs to be supplied to the cantilever for each oscillation cycle. If this energy loss is large compared to the intrinsic energy loss of the cantilever, amplitude control can become difficult [see the discussion after Eq. (47)]. A new conjecture regarding $k$ and $A$ is then

$$
\frac{k}{2} A^{2} \geqslant \Delta E_{t s} \frac{Q}{2 \pi} \text {. }
$$

The validity of these criteria is supported by an analysis of the values of $k$ and $A$ for many NC-AFM experiments with atomic resolution in Table I.

Fulfilment of the stability criteria thus requires either the use of large amplitudes, cantilevers with large spring constants, or both. However, using large amplitudes has critical disadvantages, which are discussed in Sec. VIII.

\section{B. Nonmonotonic imaging signal}

The magnitude of the tunneling current increases continuously as the tip-sample distance decreases, i.e., the tunneling current is a strictly monotonic decreasing function of the distance (see Fig. 5 in Sec. II.A.2 above). This property allows a simple implementation of a feedback loop: the tunneling current is fed into a logarithmic amplifier to produce an error signal that is linear with the tip-sample distance.

In contrast, the tip-sample force is not monotonic. In general, the force is attractive for large distances, and upon decreasing the distance between tip and sample, the force turns repulsive (see Fig. 18). Stable feedback is only possible on a branch of the force curve, where it is monotonic.

Because the tunneling current is monotonic over the whole distance range, whereas the tip-sample force is not, it is much easier to establish a $z$ distance feedback loop for an STM than for an AFM.

\section{Contribution of long-range forces}

The force between tip and sample is composed of many contributions: electrostatic, magnetic, van der Waals, and chemical forces in vacuum. In ambient conditions there are also meniscus forces. While electrostatic, magnetic, and meniscus forces can be eliminated by equalizing the electrostatic potential between tip and sample, using nonmagnetic tips, and operating in vacuum, the van der Waals forces cannot be switched off. For imaging by AFM with atomic resolution, it is desirable to filter out the long-range force contributions 
and measure only the force components that vary at the atomic scale. In an STM, the rapid decay of the tunneling current with distance naturally blocks contributions of tip atoms that are further distant from the sample, even for fairly blunt tips. In contrast, in static atomic force microscopy, long- and short-range forces add up to the imaging signal. In dynamic atomic force microscopy, attenuation of the long-range contributions is achieved by proper choice of the cantilever's oscillation amplitude $A$ (see Sec. VII.A.3).

\section{Noise in the imaging signal}

Forces can be measured by the deflection of a spring. However, measuring the deflection is not a trivial task and is subject to noise, especially at low frequencies ( $1 / f$ noise). In static atomic force microscopy, the imaging signal is given by the dc deflection of the cantilever, which is subject to $1 / f$ noise. In dynamic atomic force microscopy, the low-frequency noise is discriminated if the eigenfrequency $f_{0}$ is larger than the $1 / f$ corner frequency. With a bandpass filter with a center frequency around $f_{0}$, only the white noise density is integrated across the bandwidth $B$ of the bandpass filter.

Frequency-modulation atomic force microscopy, described in detail in Sec. VI, helps to overcome three of these four challenges. The nonmonotonic force-vsdistance relation is a remaining complication for atomic force microscopy.

\section{EARLY AFM EXPERIMENTS}

The first description of the AFM by Binnig et al. (1986) already lists several possible ways to operate the microscope: contact and noncontact, static, and dynamic modes. Initially, AFM's were mainly operated in the static contact mode. However, soon after the invention of the AFM, Dürig, Gimzewski, and Pohl (1986) measured the forces acting during tunneling in an STM in ultrahigh vacuum with a dynamic technique. In these experiments, the interaction between a tungsten STM tip and a thin film of Ag condensed on a metal cantilever was studied. The thermally excited oscillation of the metal cantilever was observed in the spectrum of the tunneling current, and the force gradient between tip and sample caused a shift in the resonance frequency of the cantilever. In a later experiment, Dürig, Züger, and Pohl (1990) used Ir tips and an Ir sample. While variations of the force on atomic scale were not reported in these experiments, it was shown that both repulsive (W tip, Ag sample) and attractive forces (Ir tip, Ir sample) of the order of a few $\mathrm{nN}$ could act during STM operation.

G. Binnig, Ch. Gerber, and others started the IBM Physics Group at the Ludwig-Maximilian-Universität in Munich. The author joined this group in May 1988 and helped to build a low-temperature ultrahigh-vacuum AFM to probe the resolution limits of the AFM. If atomic resolution was possible, we thought that the best bet would be to try it at low temperatures in order to minimize the detrimental effects of thermal noise. The microscope was fitted to a quite complex vacuum system which was designed by G. Binnig, Ch. Gerber, and T. Heppell with colleagues from VG Special Systems, Hastings, England. Because it was anticipated that the design of the instrument would have to go through many iterations involving the breaking of the vacuum, the vacuum system was designed in an effort to keep the bakeout time short and to allow rapid cooling to $4 \mathrm{~K}$ (see Giessibl, Gerber, and Binnig, 1991). Our instrument could resolve atoms in STM mode on graphite at $T$ $=4 \mathrm{~K}$ in 1989, but AFM operation with atomic resolution was not yet possible. As AFM test samples, we used ionic crystals and in particular alkali halides. Alkali halides can be viewed as consisting of hard spheres that are charged by plus/minus one elementary charge (Ashcroft and Mermin, 1981). These materials are easily prepared by cleaving in vacuum, where large [001] planes with fairly low step densities develop.

In late 1989, E. Meyer et al. (Meyer, Heinzelmann, Rudin, and Güntherodt, 1990) showed quasiatomic resolution on $\operatorname{LiF}(001)$ in ambient conditions. The AFM images were explained with the "contact-hard-spheres model" by Meyer et al. (Meyer, Heinzelmann, Brodbeck, et al., 1990), which assumes that the front atom of the tip and the sample atoms are hard spheres. Also in 1990, G. Meyer and N. M. Amer (1990) published a paper about the successful imaging of $\mathrm{NaCl}$ in ultrahigh vacuum at room temperature with quasiatomic resolution. Quasiatomic resolution means that the images reflect atomic periodicities, but no atomic defects. The images appear to arise from a tip that has several or possibly many atomic contacts (minitips) spaced by one or several surface lattice vectors. This hypothesis is supported by the common observation in contact atomic force microscopy that the resolution appears to improve after the tip is scanned for a while. Wear can cause the tip to develop a set of minitips which are spaced by multiple sample surface lattice vectors, yielding a quasiatomic resolution. This mechanism is not observed and not expected to occur in today's noncontact AFM experiments.

In both contact AFM experiments (E. Meyer et al. and G. Meyer et al.), only one type of ion was apparent in the force microscope images. In 1990, we improved our 4-K ultrahigh-vacuum instrument by mounting the whole vacuum system on air legs and adding a vibration insulation stage directly at the microscope. The major experimental challenge was the detection of the cantilever deflection. As in the first AFM by Binnig (1986), tunneling detection was used to measure the deflection of a micromachined $\mathrm{V}$-shaped cantilever with a spring constant of $k=0.37 \mathrm{~N} / \mathrm{m}$, made by Albrecht et al. (1990). The cantilever was made from $\mathrm{SiO}_{2}$ and plated with a thin gold film for electrical conductance. The tunneling tip had to be adjusted to an area of a few $\mu \mathrm{m}^{2}$ before the microscope was inserted into the low-temperature vacuum system. As it turned out later, successful tunneling between the platinum-coated tungsten tip and the gold-plated cantilever was only possible if the tip had 

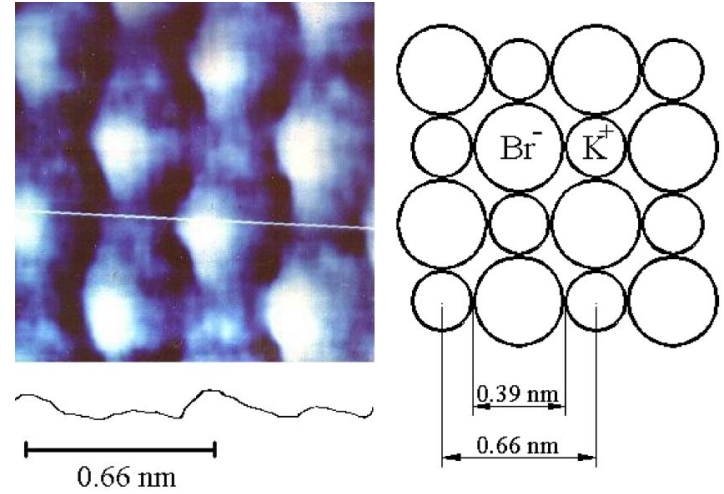

FIG. 19. (Color in online edition) Atomically resolved image of $\mathrm{KBr}(001)$ in contact AFM mode. The small and large protrusions are attributed to $\mathrm{K}^{+}$and $\mathrm{Br}^{-}$ions, respectively. From Giessibl and Binnig, 1992b.

drifted towards the fixed end of the cantilever beam during cooling of the instrument from room temperature to $4 \mathrm{~K}$. When the tunneling tip was adjacent to the free end of the cantilever, a jump-to-contact between tunneling tip and cantilever occurred and stable tunneling conditions were hard to achieve. However, if the tunneling tip met the cantilever at a distance $L_{\text {tunneling }}$ from the fixed end of the cantilever with total length $L$, the effective stiffness of the cantilever increased by a factor of $\left(L / L_{\text {tunneling }}\right)^{3}$ [see Eq. (14)] and a jump-to-contact was less likely to occur. Endurance was critical, because only in one of about ten cooling cycles did all parts of the complicated microscope work. $\mathrm{KBr}$ (cleaved in situ) was used as a sample. After the sample was approached to the cantilever, jump-to-contact occurred and the sample area where the cantilever had landed was destroyed. After jump-to-contact occurred, the pressure on the tip region was released by pulling back the sample, so that the cantilever still stayed in contact with the sample, but the repulsive force between the front atom of the cantilever and the sample was reduced to $\approx 1 \mathrm{nN}$. With the reduced tip-sample force, the sample was moved laterally to an undisturbed area, and atomic resolution was immediately obtained.

In summer of 1991, we finally succeeded in obtaining true atomic resolution on $\mathrm{KBr}$. Figure 19 shows the $\mathrm{KBr}$ (001) surface imaged in contact mode. Both ionic species are visible, because repulsive forces are used for imaging. The small bumps are interpreted as $\mathrm{K}^{+}$ions and the large bumps as $\mathrm{Br}^{-}$ions. Today even with refined noncontact AFM, only one atomic species appears as a protrusion in images of ionic crystals. Most likely, the dominant interaction between front atom and sample in noncontact AFM is electrostatic, so the charge of the front atom determines whether cations or anions appear as protrusions; see, for example, Livshits, Shluger, and Rohl (1999), Livshits et al. (1999), and Shluger et al. (1999).

Figure 20 shows atomic resolution on $\mathrm{KBr}$ with linear singularities and atomic defects. This image was obtained by scanning an area of $5 \times 5 \mathrm{~nm}^{2}$ for awhile and then doubling the scan size to $10 \times 10 \mathrm{~nm}^{2}$. The fast-

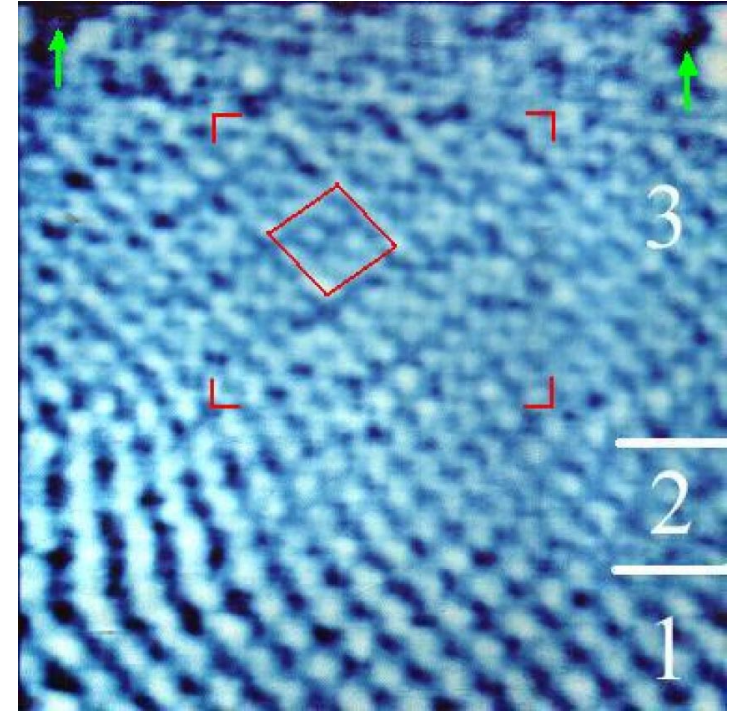

FIG. 20. (Color in online edition) Atomically resolved image of $\mathrm{KBr}(001)$ in contact AFM mode. Scan width of $5 \mathrm{~nm}$ in region 1 , is continuously increased from $5 \mathrm{~nm}$ to $10 \mathrm{~nm}$ in region 2 and $10 \mathrm{~nm}$ in region 3 (see text). The $\sqrt{3} \times \sqrt{3} R 45^{\circ}$ superstructure and the slight depression in the central $5 \mathrm{~nm}$ $\times 5 \mathrm{~nm}^{2}$ area (enclosed by angles) is probably caused by the repulsive force of $1 \mathrm{nN}$ between the front atom of the tip and the sample. The square shows the unit cell of the $\sqrt{3}$ $\times \sqrt{3} R 45^{\circ}$ reconstruction; the arrows indicate atomic-size defects. From Giessibl and Binnig, 1992a.

scanning direction was horizontal, the slow-scanning direction vertical from bottom to top. In the lower section in Fig. 20 (region 1) the scan size was $5 \times 5 \mathrm{~nm}^{2}$. Region 2 is a transition area, where the $x$ - and $y$-scan widths were continuously increased to $10 \mathrm{~nm}$ (an analog scan electronics was used where the widths of both scanning axes were independently controlled by a potentiometer in real time). In region 3, the scan size was set at 10 $\times 10 \mathrm{~nm}^{2}$. Initially, we interpreted the singularity as a monoatomic step (Binnig, 1992). However, the height difference between the central area in Fig. 20 and the surrounding area is much smaller than a single step (3.3 $\AA)$. Therefore today it appears that the central area is a "scan window," i.e., a region slightly damaged by the pressure of the scanning cantilever. Even scanning at very small loads of a $\mathrm{nN}$ or so disturbed the surface slightly and created a depressed area with a $\sqrt{3}$ $\times \sqrt{3} R 45^{\circ}$ superstructure on the $\mathrm{KBr}$ surface. The presence of atomic defects (arrows in Fig. 20), linear defects, and superstructures strengthened our confidence in the true atomic resolution capability of the AFM. However, the experimental difficulties with low-temperature operation, sample preparation, tunneling detection, etc. made it quite impractical for routine measurements.

In 1993, Ohnesorge and Binnig (1993) pursued a different method for canceling the damaging long-range forces. The long-range attractive forces which cause jump-to-contact were reduced by immersing the cantilever and sample in a liquid, as explained by Israelachvili (1991). Ohnesorge and Binnig (1993) achieved true atomic resolution by atomic force microscopy across 


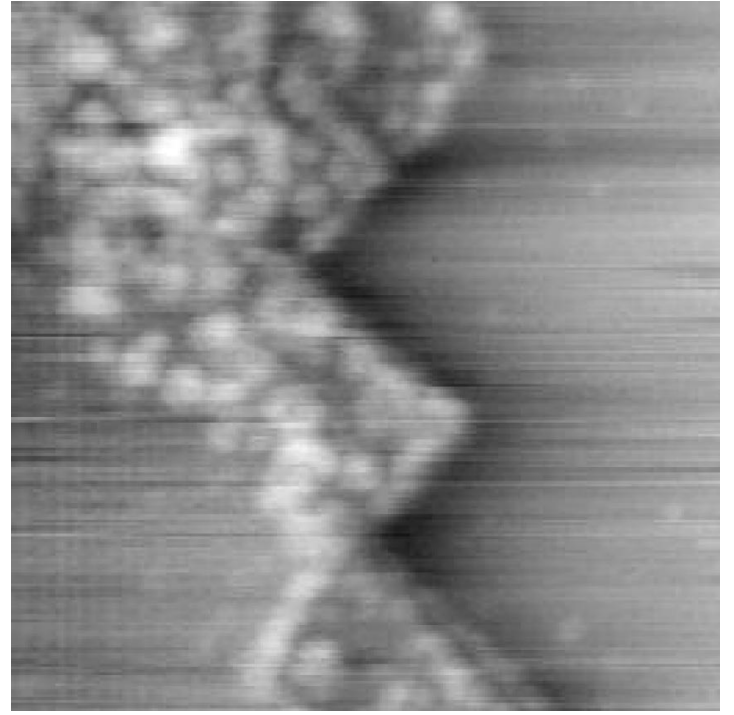

FIG. 21. Noncontact AFM image of a cleavage face of $\mathrm{KCl}$ (001) with mono- and double steps of a height of 3.1 and $6.2 \AA$, respectively. Image size, $120 \mathrm{~nm} \times 120 \mathrm{~nm}$. From Giessibl and Trafas, 1994.

steps on calcite in repulsive and attractive mode. True atomic resolution of inert surfaces by AFM had thus been clearly established. However, the enigmatic icon of atomic-resolution microscopy, $\operatorname{Si}(111)-(7 \times 7)$, remained an unsolved challenge. Even experts in experimental atomic force microscopy were convinced that this goal was impossible to reach because of silicon's high reactivity and the strong bonds that are formed between cantilever tips and the Si surface.

\section{THE RUSH FOR SILICON}

Imaging the $\mathrm{Si}(111)-(7 \times 7)$ reconstruction was crucial for the success of the STM, and therefore imaging silicon by AFM with atomic resolution was a goal for many AFM researchers. However, so far atomic resolution had not been obtained on reactive surfaces. The ions in alkali-halides form more or less closed noble-gas shells and are therefore inert. In contrast, silicon is known to form strong covalent bonds with a cohesive energy of roughly $2 \mathrm{eV}$ per bond. The jump-to-contact problem outlined in Sec. III.A is even more severe for silicon, and using silicon cantilevers on silicon samples in contact mode, in vacuum, had been proven not to work. At Park Scientific Instruments, the frequencymodulation technique pioneered by Albrecht et al. (1991) was used at that time in ambient conditions, and it was tempting to incorporate the technique into our newly designed ultrahigh-vacuum microscope (the "AutoProbe VP"). Marco Tortonese had developed piezoresistive cantilevers during his time as a graduate student in Cal Quate's group in Stanford and made them available to us. In vacuum, the piezoresistive cantilevers had excellent $Q$ values and were thus predestined for use in the FM mode. In late 1993, Giessibl and Trafas (1994) observed single steps and kinks on $\mathrm{KBr}$ using the FM

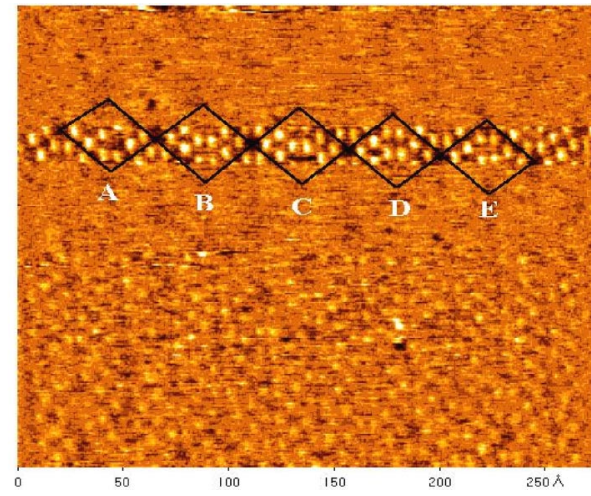

FIG. 22. (Color in online edition) First AFM image of the silicon $7 \times 7$ reconstruction with true atomic resolution. Parameters: $k=17 \mathrm{~N} / \mathrm{m}, f_{0}=114 \mathrm{kHz}, A=34 \mathrm{~nm}, \Delta f=-70 \mathrm{~Hz}$, and $Q=28$ 000. Reprinted from Giessibl, 1995. Copyright (1995) American Association for the Advancement of Science.

method (see Fig. 21). Also in 1993, Giessibl (1994) observed atomic rows on $\mathrm{Si}(111)-(7 \times 7)$, and in May 1994 (Giessibl, 1995), the first clear images of the $7 \times 7$ pattern appeared (see Fig. 22).

A different route had been pursued by the group of H.-J. Güntherodt: Howald, Lüthi, Meyer, Güthner, and Gütherodt (1994) coated the tip of the cantilever with PTFE (poly-tetra-fluoro-ethylen) and found that atomic steps and even the $\operatorname{Si}(111) 7 \times 7$ unit cell periodicity could be imaged in contact mode in vacuum (see Fig. 23). Erlandsson et al. (1997) showed that atomic resolution on $\mathrm{Si}(111) 7 \times 7$ is also possible with the amplitudemodulation technique (see Fig. 24).

\section{FREQUENCY-MODULATION ATOMIC FORCE MICROSCOPY}

\section{A. Experimental setup}

In FM-AFM, a cantilever with eigenfrequency $f_{0}$ and spring constant $k$ is subject to controlled positive feedback such that it oscillates with a constant amplitude $A$ (Albrecht et al., 1991; Dürig et al., 1992) as shown in Fig. 25. The deflection signal first enters a bandpass filter. Then the signal splits into three branches: one branch is phase shifted, routed through an analog multiplier, and fed back to the cantilever via an actuator; one branch is used to compute the actual oscillation amplitude-this signal is used to calculate a gain input $g$ for the analog multiplier; and one branch is used to feed a frequency detector. The frequency $f$ is determined by the eigenfrequency $f_{0}$ of the cantilever and the phase shift $\varphi$ between the mechanical excitation generated at the actuator and the deflection of the cantilever. If $\varphi=\pi / 2$, the loop oscillates at $f=f_{0}$.

Forces between tip and sample cause a change in $f$ $=f_{0}+\Delta f$. The eigenfrequency of a harmonic oscillator is given by $\left(k^{*} / m^{*}\right)^{0.5} /(2 \pi)$, where $k^{*}$ is the effective spring constant and $m^{*}$ is the effective mass. If the second derivative of the tip-sample potential $k_{t s}$ $=\partial^{2} V_{t s} / \partial z^{2}$ is constant for the whole range covered by the oscillating cantilever, $k^{*}=k+k_{t s}$. If $k_{t s} \ll k$, the 


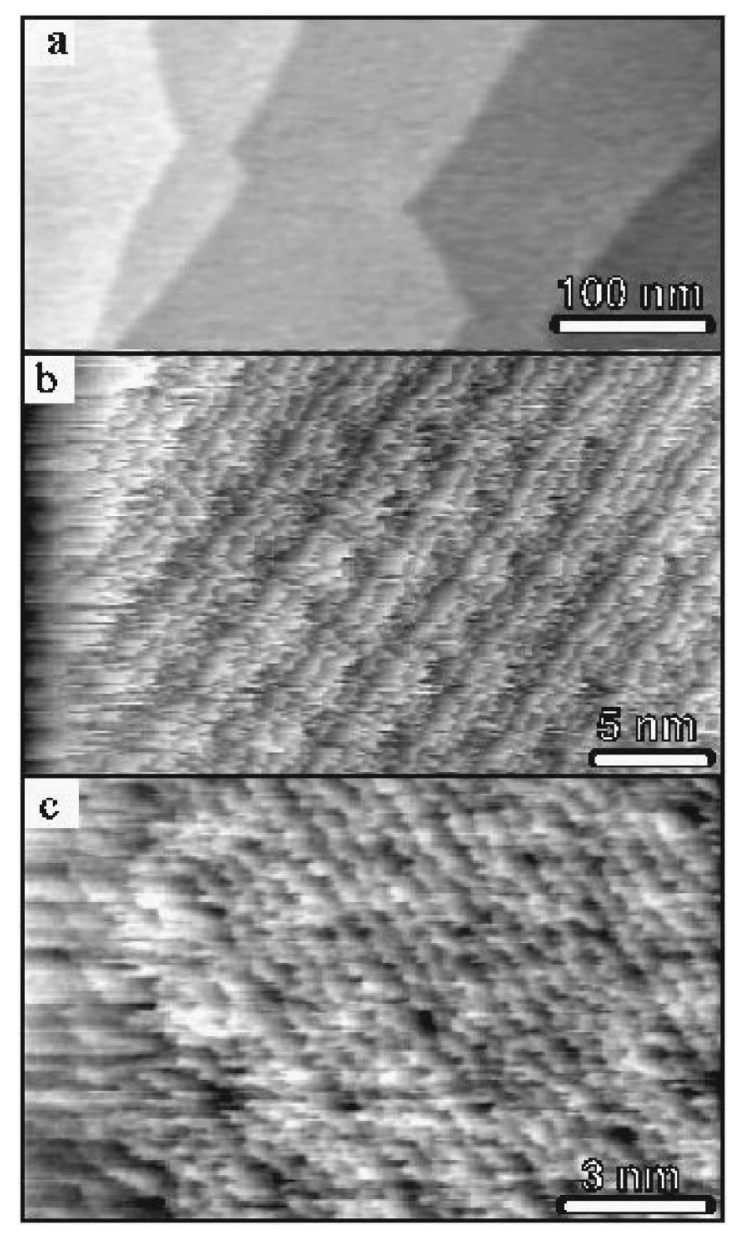

FIG. 23. Comparison of normal and lateral force microscopy images: (a) normal-force image on the surface of $\mathrm{Si}(111) 7$ $\times 7$; the step heights are 3 and $6 \AA$; (b),(c) lateral-force image on $\mathrm{Si}(111) 7 \times 7$. A repulsive force of $10^{-9} \mathrm{~N}$ is applied between the probing tip and sample. Variations of the lateral force are digitized while keeping the normal force constant. From Howald et al., 1994.

square root can be expanded as a Taylor series and the shift in eigenfrequency is approximately given by

$$
\Delta f=\frac{k_{t s}}{2 k} f_{0} .
$$

The case in which $k_{t s}$ is not constant is treated in the next section. By measuring the frequency shift $\Delta f$, one can determine the tip-sample force gradient.

The oscillator circuit is a critical component in FMAFM. The function of this device is understood best by analyzing the cantilever motion. The cantilever can be treated as a damped harmonic oscillator that is externally driven. For sinusoidal excitations $A_{\text {drive }} e^{i 2 \pi f_{\text {drive }} t}$ and a quality factor $Q \gg 1$, the response of the oscillation amplitude of the cantilever is given by

$$
\frac{A}{A_{\text {drive }}}=\frac{1}{1-f_{\text {drive }}^{2} / f_{0}^{2}+i f_{\text {drive }} /\left(f_{0} Q\right)} .
$$

The absolute value of the amplitude is given by

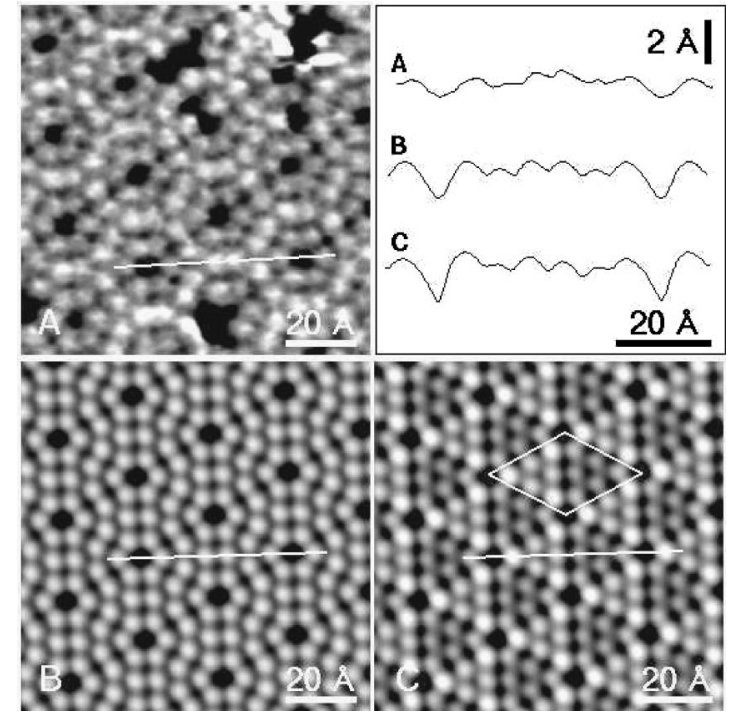

FIG. 24. AFM image of the silicon $7 \times 7$ reconstruction with amplitude-modulation (AM) mode. Image size $100 \times 100 \AA^{2}$. A comparison between (A) an AFM image and (B) emptyand (C) filled-state STM images. The gray scales in the images correspond to a height difference of $1 \AA$. The STM images were recorded with tip voltages of -2 and $+2.2 \mathrm{~V}$, respectively, and a constant current of $0.1 \mathrm{nA}$. The AFM image was low-pass filtered using a $3 \times 3$ convolution filter, while the STM images show unfiltered data. The cross sections through the four inequivalent adatoms are obtained from raw data. The 7 $\times 7$ unit cell is outlined in the filled-state STM image. The faulted and unfaulted halves correspond to the left-hand and right-hand sides, respectively. From Erlandsson et al., 1997.

$$
|A|=\frac{\left|A_{\text {drive }}\right|}{\sqrt{\left(1-f_{\text {drive }}^{2} / f_{0}^{2}\right)^{2}+f_{\text {drive }}^{2} /\left(f_{0}^{2} Q^{2}\right)}}
$$

and the phase angle between the driving and resulting signals is

$$
\varphi=\arctan \left(\frac{f_{\text {drive }}}{Q f_{0}\left(1-f_{\text {drive }}^{2} / f_{0}^{2}\right)}\right) .
$$

In the case of a closed feedback loop like that shown in Fig. 25, the driving frequency can no longer be chosen freely but is determined by $f_{0}$ of the cantilever, the phase shift $\varphi$, and the tip-sample forces. The purpose of the oscillator circuit is to provide controlled positive feedback (with a phase angle of $\varphi=\pi / 2$ ) such that the cantilever oscillates at a constant amplitude. This requirement is fulfilled with the setup shown in Fig. 25.

The cantilever deflection signal is first routed through a bandpass filter which cuts off the noise from unwanted frequency bands. The filtered deflection signal branches into an rms-to-dc converter and a phase shifter (see Horowitz and Hill, 1989). The rms-to-dc chip computes a dc signal that corresponds to the rms value of the amplitude. This signal is added to the inverted setpoint rms amplitude, yielding the amplitude error signal. The amplitude error enters a proportional $(P)$ and optional integral $(I)$ controller, and the resulting signal $g$ is multi- 


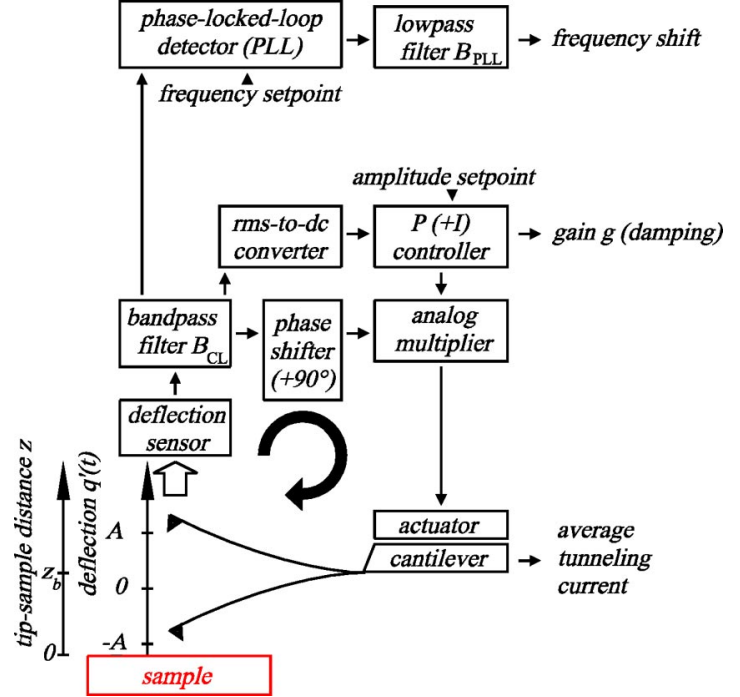

FIG. 25. (Color in online edition) Block diagram of the frequency-modulation AFM feedback loop for constant amplitude control and frequency-shift measurement. Three physical observables are available: frequency shift, damping signal, and (average) tunneling current.

plied with the phase-shifted cantilever deflection signal $q^{\prime \prime}$ with an analog multiplier chip. This signal drives the actuator. The phase shifter is adjusted so that the driving signal required for establishing the desired oscillation amplitude is minimal; $\varphi$ is exactly $\pi / 2$ in this case. Dürig et al. (1992) and Gauthier et al. (Gauthier, Sasaki, and Tsukuda; 2001; Gauthier, Perez, et al., 2002) have analyzed the stability issues related to this forced motion.

The filtered cantilever deflection signal is fed into a frequency-to-voltage converter. Initially, analog circuits were used as frequency-to-voltage converters (Albrecht et al., 1991). Recently, commercial digital phase-lockedloop (PLL) detectors made by Nanosurf, ${ }^{2}$ and analog quartz-stabilized PLL's (Kobayashi et al., 2001) became available which are more precise and more convenient. The PLL allows one to set a reference frequency $f_{\text {ref }}$ and outputs a signal that is proportional to the difference between the input frequency $f$ and the reference frequency $f_{r e f}$. This signal $\Delta f=f-f_{r e f}$ is used as the imaging signal in FM-AFM. Some researchers use the PLL's oscillator signal to drive the cantilever. The advantage is the greater spectral cleanliness of the PLL oscillator signal. A disadvantage is that the cantilever drive loop becomes more convoluted, and once the PLL is out of lock, the oscillation of the cantilever stops.

\section{B. Experimental parameters}

While it was believed initially that the net force between the front atom of the tip and the sample had to be

\footnotetext{
${ }^{2}$ These are the easyPLL Digital FM Detectors made by Nanosurf AG, Liestal, Switzerland CH-8804.
}

attractive when atomic resolution was desired, theoretical (Sokolov et al., 1999; Jarvis et al., 2001) and experimental evidence (Giessibl, Bielefeldt, et al., 2001; Giessibl, Hembacher, et al., 2001b) suggests that atomic resolution even on highly reactive samples is possible with repulsive forces. Nevertheless, the dynamic modes are commonly still called "noncontact" modes. For atomic studies in vacuum, the FM mode is now the preferred AFM technique.

FM-AFM was introduced by Albrecht, Grutter, Horne, and Rugar (1991) in magnetic force microscopy. In these experiments, Albrecht et al. imaged a thin-film CoPtCr magnetic recording disk [Fig. 7(a) in Albrecht et al. (1991)] with a cantilever that had a spring constant $k \approx 10 \mathrm{~N} / \mathrm{m}$, eigenfrequency $f_{0}=68485 \mathrm{~Hz}$, amplitude $A=5 \mathrm{~nm}, Q$ value of 40000 (Albrecht, 2000), and a tip with a thin magnetic film coverage. The noise level and imaging speed were improved significantly over those obtained by amplitude-modulation techniques. In 1993, the frequency-modulation method was implemented in the prototype of a commercial STM/AFM for ultrahigh vacuum (Giessibl and Trafas, 1994). Initial experiments on $\mathrm{KCl}$ yielded excellent resolution and, soon after, the $\operatorname{Si}(111)-(7 \times 7)$ surface was imaged with true atomic resolution for the first time (Giessibl, 1995). FM-AFM has five operating parameters:

(1) The spring constant of the cantilever $k$.

(2) The eigenfrequency of the cantilever $f_{0}$.

(3) The quality factor value of the cantilever $Q$.

(4) The oscillation amplitude $A$.

(5) The frequency shift of the cantilever $\Delta f$.

The first three parameters are determined by the type of cantilever that is used, while the last two parameters can be freely adjusted. The initial parameters which provided true atomic resolution $(k=17 \mathrm{~N} / \mathrm{m}$, $f_{0}=114 \mathrm{kHz}, Q=28000, A=34 \mathrm{~nm}, \Delta f=-70 \mathrm{~Hz}$ ) were found empirically. Surprisingly, the amplitude necessary for obtaining good results was very large compared to atomic dimensions. The necessity of using large amplitudes for obtaining good results seems counterintuitive, because the tip of the cantilever spends only a small fraction of an oscillation cycle in close vicinity to the sample. In hindsight, it appears that the large amplitudes were required to prevent instabilities of the cantilever oscillation (see Sec. III.A). Apparently, the product between spring constant and amplitude (column " $k A[\mathrm{nN}]$ " in Table I) has to be larger than $\approx 100 \mathrm{nN}$ to provide a sufficiently strong withdrawing force. In the experiments conducted in 1994 (see rows 1 and 2 in Table I), this condition was not met, and correspondingly, the resolution was not yet quite atomic. An additional lower-threshold condition for $A$ was proposed: $E=\frac{1}{2} k A^{2}$ (column " $E[\mathrm{keV}]$ " in Table I) should be large compared to $\Delta E_{t s}$ defined in Eq. (44). This condition is required for maintaining stable oscillation amplitudes, as shown below. As shown in Table I, atomic resolution on silicon and other samples was reproduced by other groups with similar operating parameters, $\Delta f \approx$ $-100 \mathrm{~Hz}, \quad k \approx 20 \mathrm{~N} / \mathrm{m}, f_{0} \approx 200 \mathrm{kHz}$, and $A \approx 10 \mathrm{~nm}$. 
Several commercial vendors now offer frequencymodulated AFM's that operate with these parameters. ${ }^{3}$

The use of high- $Q$ cantilevers with a stiffness of $k$ $\approx 20 \mathrm{~N} / \mathrm{m}$ oscillating with an amplitude of $A \approx 10 \mathrm{~nm}$ has enabled many groups to routinely achieve atomic resolution by FM-AFM. As shown in Table I, this mode is used in many laboratories now, and we therefore call it the "classic" FM-AFM mode. While the operating parameters of the classic FM-AFM mode provide good results routinely, it was not proved initially that these parameters yielded optimal resolution. The search space for finding the optimal parameters was not completely open, because micromachined cantilevers were only available with a limited selection of spring constants. A theoretical study later showed (Giessibl, Bielefeldt, et al., 1999), that the optimal amplitudes are in the $\AA$ range, requiring spring constants of the order of a few hundred $\mathrm{N} / \mathrm{m}$, much stiffer than the spring constants of commercially available cantilevers. This result has been verified experimentally by achieving unprecedented resolution with a cantilever with $k=1800 \mathrm{~N} / \mathrm{m}$ and sub-nm oscillation amplitudes (Giessibl, Hembacher, et al., 2000; Giessibl, Bielefeldt, et al., 2001). Eguchi and Hasegawa (2002) have also achieved extremely high resolution with a silicon cantilever with a stiffness of $46.0 \mathrm{~N} / \mathrm{m}$ and an oscillation amplitude of only $2.8 \mathrm{~nm}$.

\section{PHYSICAL OBSERVABLES IN FM-AFM}

\section{A. Frequency shift and conservative forces}

\section{Generic calculation}

The oscillation frequency is the main observable in FM-AFM, and it is important to establish a connection between frequency shift and the forces acting between tip and sample. While the frequency can be calculated numerically (Anczykowski et al., 1996), an analytic calculation is important for finding the functional relationships between operational parameters and the physical tip-sample forces. The motion of the cantilever (spring constant $k$, effective mass $m^{*}$ ) can be described by a weakly disturbed harmonic oscillator. Figure 26 shows the deflection $q^{\prime}(t)$ of the tip of the cantilever: it oscillates with an amplitude $A$ at a distance $q(t)$ from a sample. The closest point to the sample is $q=d$ and $q(t)=q^{\prime}(t)+d+A$. The Hamiltonian of the cantilever is

$$
H=\frac{p^{2}}{2 m^{*}}+\frac{k q^{\prime 2}}{2}+V_{t s}(q)
$$

where $p=m^{*} d q^{\prime} / d t$. The unperturbed motion is given by

$$
q^{\prime}(t)=A \cos \left(2 \pi f_{0} t\right),
$$

\footnotetext{
${ }^{3}$ These include the JEOL ultrahigh-vacuum STM AFM (JEOL Ltd., Akishima, Tokyo, Japan) and the Omicron ultrahigh-vacuum AFM/STM (Omicron Nanotechnology $\mathrm{GmbH}$, Taunusstein, Germany D-65232).
}

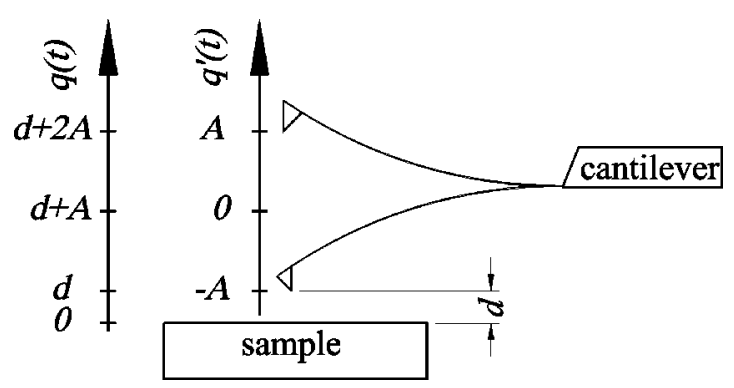

FIG. 26. Schematic view of an oscillating cantilever at its upper and lower turnaround points. The minimum tip-sample distance is $d$ and the amplitude is $A$.

and the frequency is

$$
f_{0}=\frac{1}{2 \pi} \sqrt{\frac{k}{m^{*}}} .
$$

If the force gradient $k_{t s}=-\partial F_{t s} / \partial z$ is constant during the oscillation cycle, the calculation of the frequency shift is trivial:

$$
\Delta f=f_{0} \frac{k_{t s}}{2 k} \text {. }
$$

However, in classic FM-AFM $k_{t s}$ varies by orders of magnitude during one oscillation cycle, and a perturbation approach as shown below has to be employed for the calculation of the frequency shift.

The first derivation of the frequency shift in FM-AFM (Giessibl, 1997) utilized canonical perturbation theory (see, for example, Goldstein, 1980). The result of this calculation was

$$
\Delta f=-\frac{f_{0}}{k A^{2}}\left\langle F_{t s} q^{\prime}\right\rangle
$$

where the brackets indicate averaging across one oscillation cycle.

The applicability of first-order perturbation theory depends on the magnitude of the perturbation, i.e., on the ratio between $V_{t s}$ and the energy of the oscillating cantilever $E=H_{0}$. In FM-AFM, $E$ is typically in the range of several keV's (see Table I), while $V_{t s}$ is only a few electron volts and first-order perturbation theory yields results for $\Delta f$ with excellent precision.

An alternate approach to the calculation of $\Delta f$ has been taken by Baratoff (1997), Dürig (1999a, 1999b), and Livshits, Shluger, and Rohl (1999). This approach also derives the magnitude of the higher harmonics and the constant deflection of the cantilever. The method involves solving Newton's equation of motion for the cantilever (effective mass $\mu^{*}$, spring constant $k$ ):

$$
\mu^{*} \frac{d^{2} q^{\prime}}{d t^{2}}=-k q^{\prime}+F_{t s}\left(q^{\prime}\right) .
$$

The cantilever motion is assumed to be periodic and therefore is expressed as a Fourier series with fundamental frequency $f$ :

$$
q^{\prime}(t)=\sum_{m=0}^{\infty} a_{m} \cos (m 2 \pi f t)
$$


Insertion into Eq. (30) yields

$$
\sum_{m=0}^{\infty} a_{m}\left[-(m 2 \pi f)^{2} \mu^{*}+k\right] \cos (m 2 \pi f t)=F_{t s}\left(q^{\prime}\right) .
$$

Multiplication by $\cos (l 2 \pi f t)$ and integration from $t=0$ to $t=1 / f$ yields

$$
\begin{aligned}
a_{m} & \left.-(m 2 \pi f)^{2} \mu^{*}+k\right] \pi\left(1+\delta_{m 0}\right) \\
& =2 \pi f \int_{0}^{1 / f} F_{t s}\left(q^{\prime}\right) \cos (m 2 \pi f t) d t
\end{aligned}
$$

by making use of the orthogonality of the angular functions

$$
\int_{0}^{2 \pi} \cos (m x) \cos (l x) d x=\pi \delta_{m l}\left(1+\delta_{m 0}\right) .
$$

If the perturbation is weak, $q^{\prime}(t) \approx A \cos (2 \pi f t)$ with $f$ $=f_{0}+\Delta f, f_{0}=(1 / 2 \pi) \sqrt{k / \mu^{*}}$, and $|\Delta f| \ll f_{0}$. To first order, the frequency shift is given by

$$
\begin{aligned}
\Delta f & =-\frac{f_{0}^{2}}{k A} \int_{0}^{1 / f_{0}} F_{t s}\left(q^{\prime}\right) \cos \left(2 \pi f_{0} t\right) d t \\
& =-\frac{f_{0}}{k A^{2}}\left\langle F_{t s} q^{\prime}\right\rangle,
\end{aligned}
$$

which of course equals the result of the Hamilton-Jacobi method.

The results of these calculations are also applicable for amplitude-modulation atomic force microscopy (Bielefeldt and Giessibl, 1999). Hölscher, Allers, et al. (1999) have also used a canonical perturbation-theory approach and extended it to show that the frequency shift as a function of amplitude for inverse power forces can be expressed as a rational function for all amplitudes, not just in the large-amplitude limit. Sasaki and Tsukada have obtained a similar result to Eq. (29) with a different type of perturbation theory (Sasaki and Tsukada, 1998, 1999; Tsukada et al., 2002).

2. An intuitive expression for frequency shifts as a function of amplitude

For small amplitudes, the frequency shift is a very simple function of the tip-sample forces-it is proportional to the tip-sample force gradient $k_{t s}$. For large amplitudes, the frequency shift is given by the rather complicated equations (29) and (35). With integration by parts, these complicated formulas transform into a very simple expression that resembles Eq. (28) (Giessibl, 2001):

$$
\Delta f(z)=f_{0} \frac{\left\langle k_{t s}(z)\right\rangle}{2 k}
$$

with

$$
\left\langle k_{t s}(z)\right\rangle=\frac{1}{\frac{\pi}{2} A^{2}} \int_{-A}^{A} k_{t s}\left(z-q^{\prime}\right) \sqrt{A^{2}-q^{\prime 2}} d q^{\prime} .
$$

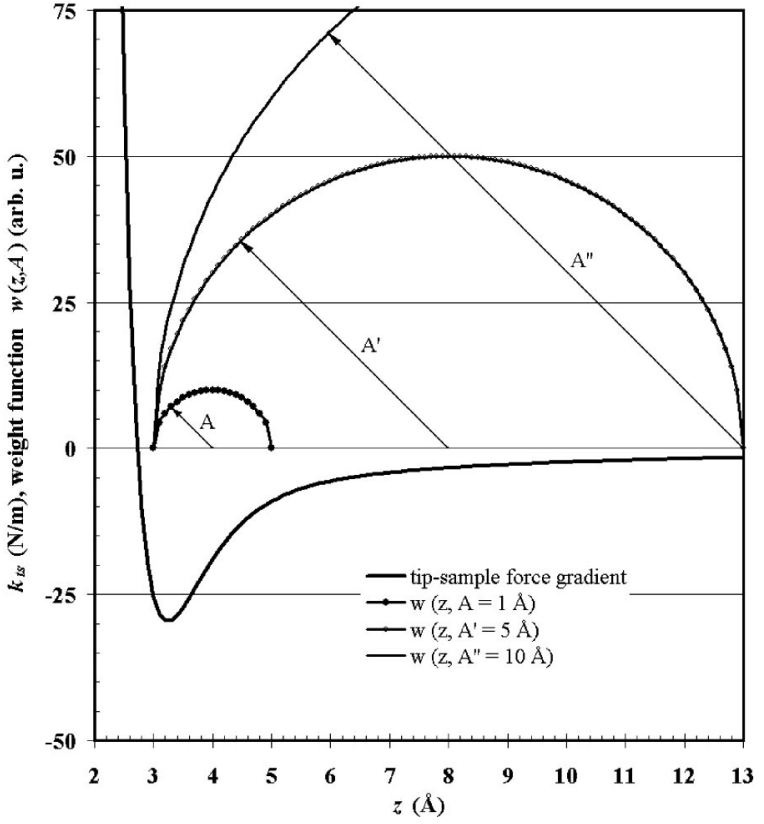

FIG. 27. Calculation of the frequency shift $\Delta f: \Delta f$ is a convolution of a semispherical weight function with the tip-sample force gradient. The radius $A$ of the weight function is equal to the oscillation amplitude of the cantilever. The weight function $w$ is plotted in arbitrary units in this scheme- $w$ has to be divided by $\pi A^{2} / 2$ for normalization (see Fig. 28).

This expression is closely related to Eq. (28): the constant $k_{t s}$ of Eq. (28) is replaced by a weighted average $\left\langle k_{t s}\right\rangle$, where the weight function $w\left(q^{\prime}, A\right)$ is a semicircle with radius $A$ divided by the area of the semicircle $\Gamma$ $=\pi A^{2} / 2$ (see Fig. 27). For $A>0$, the semicircular weight function with its normalization factor $2 / \pi A^{2}$ is a representation of Dirac's delta function. Figure 28 shows the convolution with the proper normalization factor, and it is immediately apparent from this figure how the use of small amplitudes increases the weight of the short-range atomic forces over the unwanted long-range forces. The amplitude in FM-AFM allows one to tune the sensitivity of the AFM to forces of various ranges.

\section{Frequency shift for a typical tip-sample force}

The interaction of the macroscopic tip of an AFM with a sample is a complicated many-body problem, and $F_{t s}$ cannot be described by a simple function. However, quite realistic model forces can be constructed from linear combinations of the following basic types: (a) inverse-power forces, (b) power forces, and (c) exponential forces. Analytic expressions for the frequency shift as a function of tip-sample distance $z$ and amplitude $A$ are listed in Giessibl and Bielefeldt (2000). A typical tip-sample force is composed of long-range contributions and short-range contributions. This force can be approximated by a long-range van der Waals component and a short-range Morse-type interaction:

$$
F_{t s}(z)=\frac{C}{z+\sigma}+2 \kappa E_{\text {bond }}\left(-e^{-\kappa(z-\sigma)}+e^{-2 \kappa(z-\sigma)}\right) .
$$




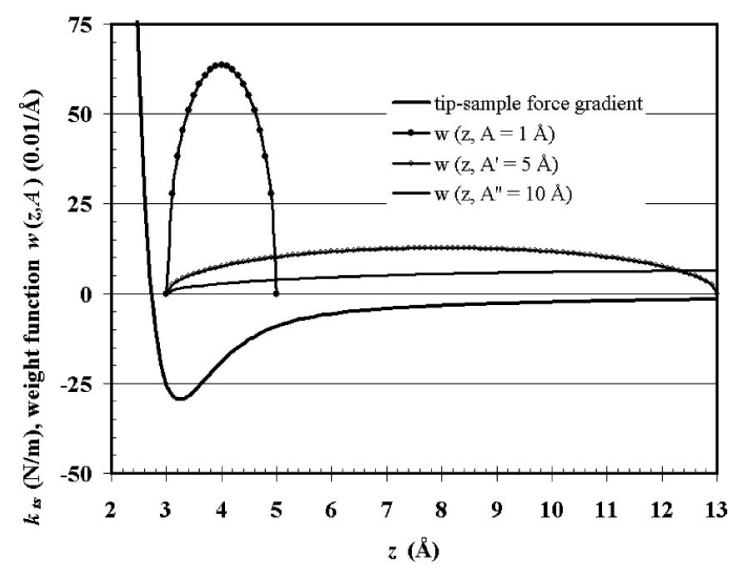

FIG. 28. Calculation of the frequency shift $\Delta f: \Delta f$ is a convolution of a weight function $w$ with the tip-sample force gradient. For small amplitudes, short-range interactions contribute heavily to the frequency shift, while long-range interactions are attenuated. However, for large amplitudes the long-range interactions cause the main part of the frequency shift, and short-range interactions play a minor role.

Here $C$ depends on the tip angle and the Hamaker constant of tip and sample, and $E_{\text {bond }}, \sigma$, and $\kappa$ are the bonding energy, equilibrium distance, and decay length of the Morse potential, respectively. With the results derived in Giessibl and Bielefeldt (2000), the resulting frequency shift is

$$
\begin{aligned}
\Delta f(z, A)= & \frac{f_{0}}{k A} \frac{C}{z+\sigma}\left[F_{1}^{1,1 / 2}\left(\frac{-2 A}{z+\sigma}\right)-F_{2}^{1,3 / 2}\left(\frac{-2 A}{z+\sigma}\right)\right] \\
& -f_{0} \frac{2 \kappa E_{\text {bond }}}{k A}\left\{e^{-\kappa z}\left[M_{1}^{1 / 2}(-2 \kappa A)-M_{2}^{3 / 2}(-2 \kappa A)\right]\right. \\
& \left.+e^{-2 \kappa z}\left[M_{1}^{1 / 2}(-4 \kappa A)-M_{2}^{3 / 2}(-4 \kappa A)\right]\right\}, \quad \text { (39) }
\end{aligned}
$$

where $F_{c}^{a, b}(z)$ is the hypergeometric function and $M_{b}^{a}(z)$ is Kummer's function (Abramowitz and Stegun, 1970).

Equation (39) describes the frequency shift as a function of amplitude and tip-sample distance. For small amplitudes, the frequency shift is independent of the amplitude and proportional to the tip-sample force gradient $k_{t s}$ [Eq. (28)]. For amplitudes that are large compared to the range of the tip-sample force, the frequency shift is a function of the amplitude, $\Delta f \propto A^{-1.5}$. If amplitudes larger than the range of the relevant forces are used, it is helpful to introduce a "normalized frequency shift" $\gamma$ defined by

$$
\gamma(z, A):=\frac{k A^{3 / 2}}{f_{0}} \Delta f(z, A) .
$$

For large amplitudes, $\gamma(z, A)$ asymptotically approaches a constant value (see Fig. 2 in Giessibl and Bielefeldt, 2000), i.e., $\lim _{A \rightarrow \infty} \gamma(z, A) \equiv \gamma_{l A}(z)$. The normalized frequency shift is calculated from the tip-sample force with

$$
\gamma_{l A}(z)=\frac{1}{\sqrt{2} \pi} \int_{0}^{\infty} \frac{F_{t s}\left(z+z^{\prime}\right)}{\sqrt{z^{\prime}}} d z^{\prime} .
$$

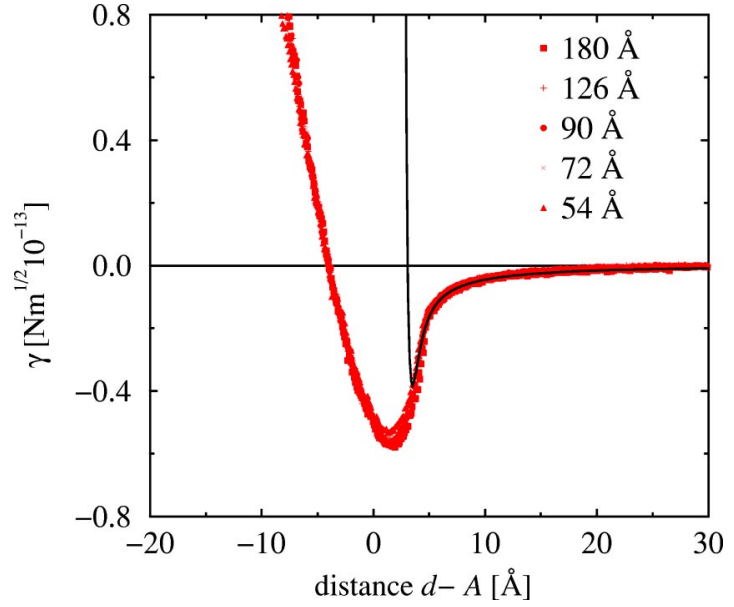

FIG. 29. (Color in online edition) Experimental normalized frequency-shift versus distance data, acquired with a lowtemperature ultrahigh-vacuum AFM with a graphite sample surface and a silicon cantilever. The average distance between the center of the tip's front atom and the plane defined by the centers of the surface atom layer is $d$, thus the minimal tipsample distance is $d-A$. Five experimental frequency-shift versus distance data sets with amplitudes from 54 to $180 \AA$ are expressed in the normalized frequency shift $\gamma=k A^{3 / 2} \Delta f / f_{0}$. The five experimental data sets match exactly, proving the validity of the concept of a normalized frequency shift. The black curve is a simulated $\gamma(d-A)$ curve using a Lennard-Jones short-range force and a $1 /(d-A)$ long-range force. In the repulsive regime, the deviation between the experimental dots and the simulated curve is substantial, because the $1 /(d$ $-A)^{12}$ dependence of the repulsive Lennard-Jones potential describes only the interaction of the tip and sample atom. The sample atom is embedded in a lattice with finite stiffness; graphite, the sample surface material, is a very soft material. Hertzian contact theory (see, for example, Chen, 1993) is an appropriate model when the repulsive forces are large enough to cause overall sample deformations. From Hölscher et al., 2000.

The normalized frequency shift helps to characterize AFM experiments and has a similar role as the tunneling impedance in scanning tunneling microscopy on metals. Hölscher et al. (2000) have performed frequency-shift versus distance measurements with a silicon cantilever on a graphite surface, with amplitudes ranging from 54 to $180 \AA$, and verified the concept of the normalized frequency shift $\gamma=\Delta f \times k \times A^{3 / 2} / f_{0}$ as the pertinent imaging parameter in classic FM-AFM (see Fig. 29). Five frequency-shift curves taken with amplitudes ranging from 54 to $180 \AA$ match precisely when rescaled using the normalized frequency shift. Thus for small amplitudes the frequency shift is very sensitive to short-range forces, because short-range forces have a very strong force gradient, while for large amplitudes, long-range forces contribute heavily to the frequency shift. Figure 30 shows the tip-sample force defined in Eq. (38) and the corresponding force gradient and normalized frequency shift $\gamma_{l A}$. The parameters for the short-range interaction are adopted from Perez et al. (1998): $\kappa$ $=12.76 \mathrm{~nm}^{-1}, E_{\text {bond }}=2.273 \mathrm{eV}$, and $\sigma=2.357 \AA$. The 


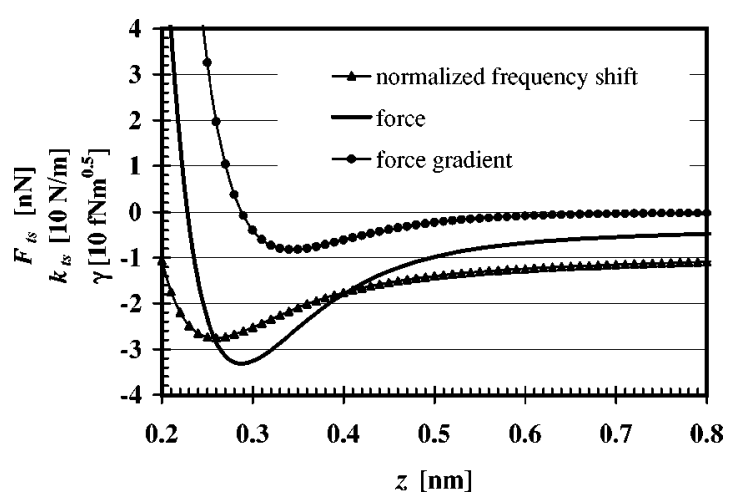

FIG. 30. Force $F_{t s}(z)$, force gradient $k_{t s}(z)$, and largeamplitude normalized frequency shift $\gamma_{l A}(z)$ for the tipsample force defined in Eq. (38). When the cantilever's oscillation amplitude $A$ is small compared to the range of the tipsample forces $\lambda$, the frequency shift is proportional to the force gradient, while for $A \gg \lambda$, the frequency shift is proportional to $\gamma_{l A}(z)$.

force gradient is vanishing for $z>6 \AA$, while the normalized frequency shift for large amplitudes reaches almost half its maximum at this distance. The dependence of the frequency shift on amplitude shows that small amplitudes increase the sensitivity to short-range forces! The ability to adjust the amplitude in FM-AFM compares to tuning an optical spectrometer to a passing wavelength. When short-range interactions are to be probed, the amplitude should be in the range of the short-range forces. While using amplitudes in the $\AA$ range has been problematic with conventional cantilevers because of the instability problem described in Sec. III.A, stiff sensors such as the qPlus sensor displayed in Fig. 11 are well suited for small-amplitude operation.

\section{Deconvolution of forces from frequency shifts}

Frequency shifts can be measured with high accuracy and low noise, while the measurement of dc forces is subject to large noise. However, forces and not frequency shifts are of primary physical interest. A number of methods have been proposed to derive forces from the frequency shift curves.

The first type of method requires the relation of frequency shift versus distance $\Delta f(z)$ over the region of interest. Because force and frequency shift are connected through a convolution, a deconvolution scheme is needed to connect forces (or force gradients) to the frequency shift and vice versa. Giessibl (1997) has proposed constructing a model force composed from basic functions (inverse power and exponential forces) and fitting the parameters (range and strength) of the model force so that its corresponding frequency shift matches the experimental frequency shift. Gotsmann et al. (1999) have proposed a numerical algorithm, and Dürig (1999b) has developed an iterative scheme for force deconvolution. Giessibl (2001) has proposed a simple and intuitive matrix method to deconvolute forces from frequency shifts.

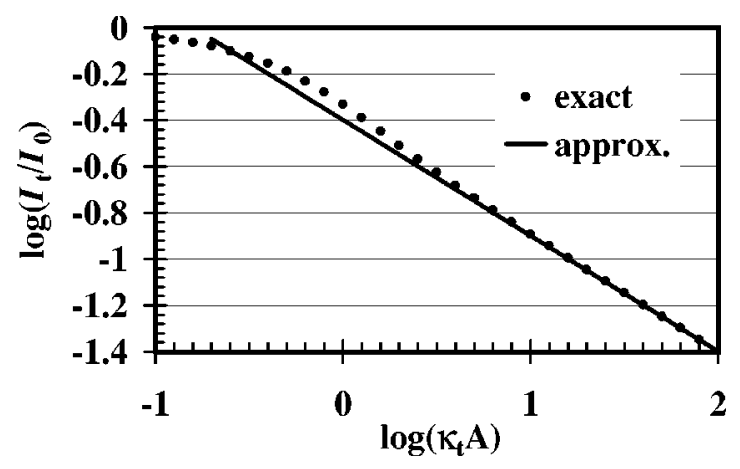

FIG. 31. Averaged tunneling current as a function of amplitude for a fixed minimum tip-sample distance.

The second type of spectroscopy method requires a knowledge of the frequency as a function of cantilever amplitude $\Delta f(A)$. Hölscher, Allers, et al. (1999) and Hölscher (2002) have modified the method elucidated in Sec. 12 of Landau's textbook on classical mechanics (Landau and Lifshitz, 1990, 13th ed.) to recover the interaction potential from the dependence of the oscillation period $T=1 / f$ on energy $E=k A^{2} / 2$.

For the third approach, developed by Dürig (2000), the full tip-sample potential curve can be recovered within the $z$ interval covered by the cantilever motion if the amplitudes and phases of all the higher harmonics of the cantilever motion are known. This method is very elegant because, in principle, the higher harmonics can be measured in real time, which eliminates the need to take time-consuming $\Delta f(z)$ or $\Delta f(A)$ spectra. Dürig's method is particularly promising for small-amplitude operations, because already the first few harmonics at $2 f, 3 f, \ldots$ contain characteristic information about the tip-sample potential.

\section{B. Average tunneling current for oscillating tips}

When the tip of the cantilever and the sample are both conductive, simultaneous STM and FM-AFM operation is possible, i.e., the tunneling current $I_{t}$ as well as the frequency shift can be recorded while scanning the surface. In most cases, the bandwidth of the tunneling current preamplifier is much smaller than the oscillation frequency $f_{0}$ of typical cantilevers. The measured tunneling current is given by the time-average over one oscillation cycle. With the exponential distance dependence $I_{t}(z)=I_{0} e^{-2 \kappa_{t} z}$ [see Eq. (1)] we find

$$
\left\langle I_{t}(z, A)\right\rangle=I_{0} e^{-2 \kappa_{t} z} M_{1}^{1 / 2}\left(-4 \kappa_{t} A\right),
$$

where $M_{b}^{a}(\zeta)$ is the Kummer function (Abramowitz and Stegun, 1970). When $\kappa_{t} A \gg 1$,

$$
\left\langle I_{t}(z, A)\right\rangle \approx I_{t}(z, 0) / \sqrt{4 \pi \kappa_{t} A} .
$$

Figure 31 shows the tunneling current as a function of the product between $\kappa_{t}$ and $A$. For $A=5 \mathrm{~nm}$ and $\kappa_{t}$ $=1 \AA^{-1}$, the average tunneling current is $\approx 1 / 25$ of the value when the cantilever does not oscillate. Because the noise of the current measurement decreases with an in- 
creasing average tunneling current, the use of small amplitudes improves the quality of simultaneous STM and FM-AFM measurements.

It should be noted that Eq. (43) is an upper threshold. When using large amplitudes $\left(\kappa_{t} A \gg 1\right)$, the tunneling current vs time is a series of Gaussian functions spaced by $1 / f$ where $f$ is the oscillation frequency of the cantilever. Especially when using cantilevers with large eigenfrequencies, the tunneling current varies very rapidly with time. Because of slew-rate and bandwidth limitations, typical tunneling preamplifiers are unable to convert these rapid current variations in output voltage swings. Thus the experimental average current can become even smaller than that given by Eq. (43).

\section{Damping and dissipative forces}

Conservative tip-sample forces cause a frequency shift. A nonconservative component in the tip-sample force, that is, a hysteresis in the force-versus-distance graph

$$
\Delta E_{t s}(\vec{x})=\oint_{\Lambda} \vec{F}_{t s}\left(\vec{x}+\vec{x}^{\prime}\right) d \vec{x}^{\prime},
$$

where $\Lambda$ is the trajectory of the oscillating cantilever, causes energy loss in the motion of the cantilever. This energy loss is measurable. The cantilever itself already dissipates energy (internal dissipation). When the tip of the cantilever is far from the sample, the damping of the cantilever is due to internal dissipation and the energy loss per oscillation cycle is given by

$$
\Delta E_{C L}=2 \pi \frac{E}{Q},
$$

where $E=k A^{2} / 2$ is the energy of the cantilever and $Q$ is its quality factor. When the phase angle between the excursion of the actuator and the excursion of the cantilever is exactly $\varphi=\pi / 2$, the cantilever oscillates at frequency $f_{0}$ and the driving signal is $A_{\text {drive }}=A e^{i \pi / 2} / Q$. Hence the driving amplitude and dissipation are connected:

$$
\left|A_{\text {drive }}\right|=|A| \frac{\Delta E_{C L}}{2 \pi E} .
$$

When the tip oscillates close to the sample, additional damping occurs and the driving signal $A_{\text {drive }}$ is increased by the oscillator control electronics to $A_{\text {drive }}^{\prime}$ in order to maintain a constant amplitude $A$ where

$$
\left|A_{\text {drive }}^{\prime}\right|=|A| \frac{\Delta E_{C L}+\Delta E_{t s}}{2 \pi E}=|A|\left(\frac{1}{Q}+\frac{\Delta E_{t s}}{2 \pi E}\right) .
$$

Equation (47) has an important implication for the optimal $Q$ factor of the cantilever. While a high $Q$ factor results in low-frequency noise [see Eq. (50) below], Eq. (47) shows that the $Q$ value of the cantilever should not be much higher than the ratio $2 \pi E / \Delta E_{t s}$. If $Q$ is much higher than this value, it is difficult for the oscillator circuit to maintain a constant amplitude, because small changes in $\Delta E_{t s}$ require a major correction in the control output $g$.

Measurement of the damping signal yields the dissipation in the approach and retract phases of the oscillating tip, where

$$
\Delta E_{t s}=2 \pi \frac{E}{Q}\left(\frac{\left|A_{\text {drive }}^{\prime}\right|}{\left|A_{\text {drive }}\right|}-1\right) .
$$

The ratio $\left|A_{\text {drive }}^{\prime}\right| /\left|A_{\text {drive }}\right|$ is easily accessible in the dc input $(g)$ of the analog multiplier chip shown in Fig. 25 -an increase in the tip-sample dissipation $\Delta E_{t s}$ is reflected in an increased gain signal $g^{\prime}$ in the oscillator electronics and $g^{\prime} / g=\left|A_{\text {drive }}^{\prime}\right| /\left|A_{\text {drive }}\right|$. Several authors have recorded this signal simultaneously with the frequency shift and thus measured both elastic and nonelastic interaction forces simultaneously. See, for example, Bammerlin et al. (1997); Luthi et al. (1997); Ueyama et al. (1998); Hug and Baratoff (2002).

Physical origins of dissipation are discussed by Abdurixit et al. (1999); Dürig (1999a); Hoffmann, Jeffery, et al. (2001); Gauthier, Kantorovich, et al. (2002); Giessibl, Herz, and Mannhart (2002); and Hug and Baratoff (2002).

It should also be noted that dispersions in the oscillator circuit and in the actuator assembly can lead to artifacts in the interpretation of damping data, because $\left|A_{\text {drive }}\right|=|A| / Q$ only holds for $f=f_{0}$. Anczykowski et al. (1999) have introduced a method that yields the correct dissipation energy even for cases in which the phase angle between actuator and cantilever is not $\varphi=\pi / 2$.

Mechanical resonances in the actuator assembly are likely to occur at the high resonance frequencies of conventional cantilevers. These resonances can cause sharp variations of the phase with frequency and thus create artifacts in the measurement of $\Delta E_{t s}$. A self-oscillation technique for cantilevers (Giessibl and Tortonese, 1997) helps to avoid these resonances.

\section{NOISE IN FREQUENCY-MODULATION ATOMIC FORCE MICROSCOPY}

\section{A. Generic calculation}

The vertical noise in FM-AFM can be calculated in the same fashion as in the STM case (see Fig. 5); it is given by the ratio between the noise in the imaging signal and the slope of the imaging signal with respect to $z$ :

$$
\delta z=\frac{\delta \Delta f}{\left|\frac{\partial \Delta f}{\partial z}\right|} .
$$

Figure 32 shows a typical frequency-shift-versus-distance curve. Because the distance between the tip and sample is measured indirectly through the frequency shift, it is clearly evident that the noise in the frequency measurement $\delta f=\delta \Delta f$ translates into vertical noise $\delta z$ and is given by the ratio between $\delta \Delta f$ and the slope of the frequency shift curve $\Delta f(z)$ [Eq. (49)]. Low vertical 


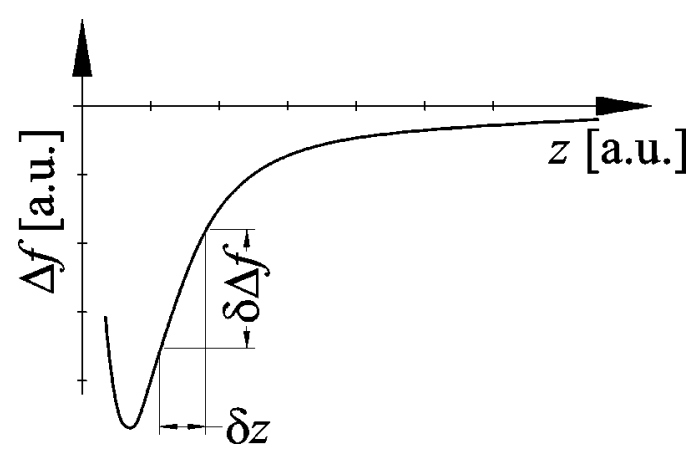

FIG. 32. Schematic plot of frequency shift $\Delta f$ as a function of tip-sample distance $z$. The noise in the tip-sample distance measurement is given by the noise of the frequency measurement $\Delta f$ divided by the slope of the frequency-shift curve.

noise is obviously obtained for a low-noise frequency measurement and a steep slope of the frequency-shift curve. Additional boundary conditions apply: if the force between front atom and the surface is too large, the front atom or larger sections of tip or sample can shear off. It is interesting to note that in FM-AFM the noise will increase again upon further reducing the tipsample distance when approaching the minimum of the $\Delta f(z)$ curve. Because the frequency shift is not monotonic with respect to $z$, stable feedback of the microscope is only possible either on the branch of $\Delta f$ with positive slope or on the one with negative slope. In FMAFM with atomic resolution, the branch with positive slope is usually chosen. However, when using very small amplitudes, it is also possible to work on the branch with negative slope (see Giessibl, Hembacher, et al., 2001b).

It is of practical importance to note that the minimum of the frequency-versus-distance curve shown in Fig. 32 is a function of the lateral tip position. Directly over a sample atom, the minimum can be very deep. However, at other sample sites there may be small negative frequency shift. Imaging can only be performed with frequency-shift setpoints that are reachable on every $(x, y)$ position on the imaged sample area, otherwise a tip crash occurs.

\section{B. Noise in the frequency measurement}

Equation (49) shows that the accuracy of the frequency-shift measurement determines directly the vertical resolution in FM-AFM. What is the accuracy of the measurement of the oscillation frequency of the cantilever? Martin et al. (1987) and McClelland et al. (1987) have studied the influence of thermal noise on the cantilever, and Albrecht et al. (1991) and Smith (1995) have calculated the thermal limit of the frequency noise. Leaving aside prefactors of the order of $\pi$, all these authors come to a similar conclusion, namely, that the square of the relative frequency noise is given by the ratio between the thermal energy of the cantilever $\left(k_{B} T\right)$ and the mechanical energy stored in it $\left(0.5 k A^{2}\right)$, divided by its quality factor $Q$ and multiplied by the

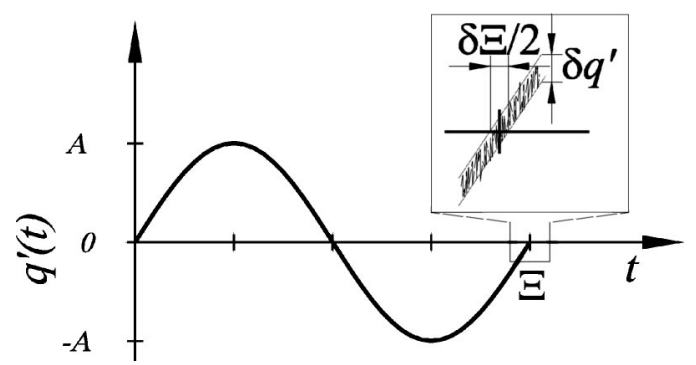

FIG. 33. Typical cantilever deflection signal as it appears on an oscilloscope. The oscillation frequency is given by the inverse time lag between two consecutive zero crossings with positive velocity.

ratio between bandwidth $B$ and cantilever eigenfrequency $f_{0}$. Specifically, Albrecht et al. (1991) find

$$
\frac{\delta f_{\text {thermal }}}{f_{0}}=\sqrt{\frac{k_{B} T B}{\pi k A^{2} f_{0} Q}} .
$$

Albrecht et al. (1991) support Eq. (50) with measurements on the dependence of $\delta f_{0}$ upon $Q$ (Fig. 5 in Albrecht et al., 1991) and $A$ (Fig. 6 in Albrecht et al., 1991) and clearly state that Eq. (50) contains only the thermal cantilever noise and disregards the noise of the deflection sensor. Correspondingly, the frequency noise becomes larger than predicted by Eq. (50) for large $Q$ values in Fig. 5 of Albrecht et al. (1991). These deviations are traced to interferometer noise, e.g., noise in the cantilever's deflection sensor. Albrecht et al. (1991) do not provide measurements of $\delta f_{0}$ as a function of bandwidth $B$. Equation (50) predicts a $B^{0.5}$ dependence. However, theoretical arguments by Dürig et al. (1997) and an analysis and measurements by Giessibl (2002) indicate a $B^{1.5}$ dependence of frequency noise. The following analysis shows the reasons for that.

The frequency is given by the inverse of the time lag $\Xi$ between two consecutive zero crossings of the cantilever with positive velocity. However, the deflection of the cantilever $q^{\prime}$ is subject to a noise level $\delta q^{\prime}$ as shown in Fig. 33. The deflection noise $\delta q^{\prime}$ has two major contributions: thermal excitation of the cantilever outside of its resonance frequency and instrumental noise in the measurement of the deflection $q^{\prime}$. The oscillation period $\Xi$ can only be measured with an rms accuracy $\delta \Xi$. The uncertainty of the time of the zero crossing is $\delta \Xi / 2$, where $\delta \Xi / 2$ is given by the ratio between the cantilever deflection noise and the slope of the $q^{\prime}(t)$ curve:

$$
\frac{\delta \Xi}{2}=\frac{\delta q^{\prime}}{2 \pi f_{0} A} .
$$

Because $f_{0}=1 / \Xi, \delta f_{0} / f_{0}=\delta \Xi / \Xi$ and

$$
\frac{\delta f_{\text {detector }}}{f_{0}}=\frac{\delta q^{\prime}}{\pi A} \text {. }
$$

Equation (52) applies only to frequency changes on a time scale of $1 / f_{0}$. When measuring frequency variations over a longer time scale, more zero crossings can be used to determine the frequency change, and the precision of the frequency measurement increases. The out- 
put of the frequency detector (phase-locked-loop, see Fig. 25) typically has a low-pass filter with bandwidth $B_{F M} \ll f_{0}$; thus the effective frequency noise is smaller than the value after Eq. (52) (see Giessibl, 2002). With $\delta q^{\prime}=n_{q^{\prime}} \sqrt{B_{F M}}$, we find

$$
\delta f_{\text {detector }}=\frac{n_{q^{\prime}}}{\pi A} B_{F M}^{3 / 2} .
$$

The scaling law $\delta f \propto B_{F M}^{3 / 2}$ was first found by Dürig et al. (1997). The frequency noise has two major contributors: (a) thermal noise of the cantilever and (b) detector noise. Because the two noise sources are statistically independent, we find (Becker, 1969)

$$
\delta f=\sqrt{\delta f_{\text {detector }}^{2}+\delta f_{\text {thermal }}^{2}}
$$

with

$$
\delta f_{\text {thermal }}=\sqrt{k_{B} T B f_{0} /\left(\pi k A^{2} Q\right)} .
$$

The detector deflection noise density $n_{q^{\prime} \text { detector }}$ is determined by the physical setup of the deflection sensor and describes the quality of the deflection sensor. For practical purposes, it can be assumed to be constant for frequencies around $f_{0}$. Good interferometers reach deflection noise densities of $100 \mathrm{fm} / \sqrt{\mathrm{Hz}}$. For most setups, thermal noise is negligible compared to detector noise.

In summary, the frequency noise is proportional to the deflection noise density times $B^{1.5}$ and inversely proportional to the amplitude. While Eq. (55) suggests the use of cantilevers with infinitely high $Q$, Eq. (47) and the discussion after it imply that $Q$ should not be significantly larger than the ratio between the energy stored in the cantilever and the energy loss per oscillation cycle due to the tip-sample interaction. If $Q$ is much higher than this value, controlling the amplitude of the cantilever can become difficult, and instabilities are likely to occur. Frequency noise is discussed in greater depth and compared to experimental noise measurements by Giessibl (2002).

\section{Optimal amplitude for minimal vertical noise}

Both the nominator and denominator in the generic FM-AFM noise [Eq. (49)] are functions of the amplitude-the frequency noise is proportional to $1 / A$, the slope of the frequency shift curve is constant at first and drops as $A^{-1.5}$ for large amplitudes. Thus there is minimal noise for amplitudes on the order of the range $\lambda$ of the tip sample force $F_{t s}$ (Giessibl, Bielefeldt, et al., 1999):

$$
A_{\text {optimal }} \approx \lambda \text {. }
$$

Here, we calculate the vertical noise for a specific example. We consider a tip-sample interaction given by a Morse potential with a depth of $-2.15 \mathrm{eV}$, a decay length of $\kappa=1.55 \AA^{-1}$, and an equilibrium distance of $\sigma=2.35 \AA$. As a cantilever, we consider a qPlus sensor as shown in Fig. 11 with $k=1800 \mathrm{~N} / \mathrm{m}$ and $n_{q^{\prime}}$ $=100 \mathrm{fm} / \sqrt{\mathrm{Hz}}$, operated with $B_{F M}=100 \mathrm{~Hz}$. Figure 34 shows the vertical noise as a function of amplitude for a fixed closest tip-sample distance of $z_{\min }=4 \AA$. Minimum

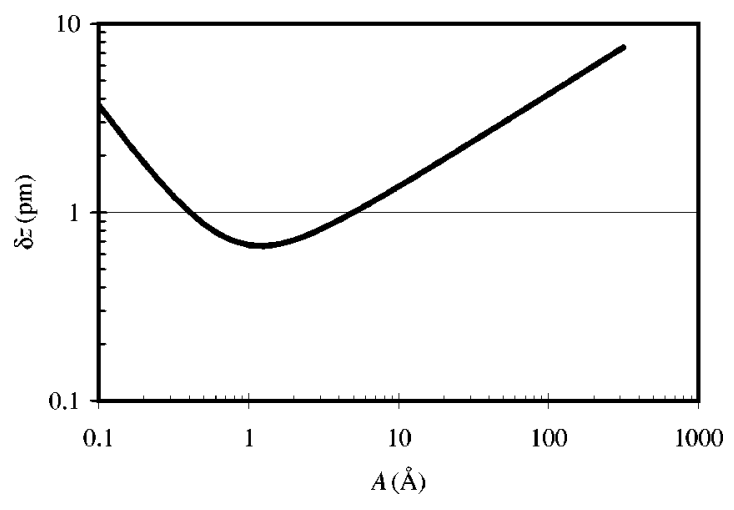

FIG. 34. Vertical noise as a function of amplitude for the tipsample potential (Morse type) described in the text. The amplitude value where minimal noise results (here $A_{\text {optimal }}$ $\approx 1 \AA$ ) is approximately equal to the range of the tip-sample force. The absolute noise figure for this optimal amplitude is a function of bandwith, noise performance of the cantilever deflection sensor, and temperature.

noise occurs for $\log A / \mathrm{m}=-9.9$, i.e., for $A \approx 1.26 \AA$, while for $A=340 \AA$ the noise is about one order of magnitude larger. For chemical forces, $\lambda \approx 1 \AA$. However, operating a conventional cantilever with amplitudes in the $\AA$ range close to a sample is impossible because of the jump-to-contact problem (Sec. III.A). The cantilever spring constant $k$ needs to be at least a few hundred $\mathrm{N} / \mathrm{m}$ to enable operation with amplitudes in the $\AA$ range.

\section{APPLICATIONS OF CLASSIC FREQUENCY- MODULATION ATOMIC FORCE MICROSCOPY}

\section{A. Imaging}

Shortly after the first demonstration of true atomic resolution of Si by AFM, Kitamura and Iwatsuki (1995); Güthner (1996); Lüthi et al. (1996); and Nakagiri et al. (1997) succeeded in imaging Si with atomic resolution using FM-AFM with similar parameters. In November 1994, Patrin (1995) succeeded in imaging $\mathrm{KCl}$, an insulator with FM-AFM (see Fig. 35). Other semiconductors (Sugawara et al., 1995; Morita and Sugawara, 2002), more ionic crystals (Bammerlin et al., 1997; Reichling and Barth, 1999, 2002; Bennewitz, Bammerlin, and Meyer, 2002), metal oxides (Fukui et al., 1997; Raza et al., 1999; Barth and Reichling, 2002; Fukui and Iwasawa, 2002; Hosoi et al., 2002; Pang and Thornton, 2002), metals (Loppacher et al., 1998; Minobe et al., 1999), organic monolayers (Gotsmann et al., 1998; Yamada, 2002), adsorbed molecules (Sasahara and Onishi, 2002; Sugawara, 2002), and even a film of xenon physisorbed on graphite (Allers et al., 1998; see Fig. 36) were imaged with atomic resolution. FM-AFM can also be used for high-resolution Kelvin probe microscopy by studying the influence of electrostatic forces on the image (Kitamura and Iwatsuki, 1998; Arai and Tomitori, 2002). Classic frequency-modulation atomic force microscopy provided a new tool to study problems that were not accessible by STM. Sugawara et al. (1995; see 


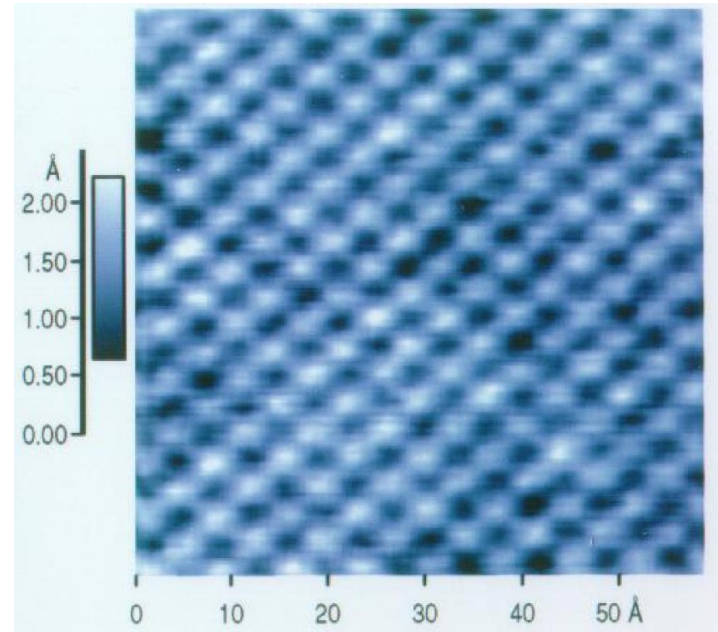

FIG. 35. (Color in online edition) First frequency-modulated atomic force microscopy (FM-AFM) image of an insulator $(\mathrm{KCl})$ with true atomic resolution. Instrument and parameters similar to Fig. 22. From Patrin, 1995.

Fig. 37) imaged defects in InP. While InP could be imaged by STM's, the bias voltage required in an STM caused the defects to move. By FM-AFM, a zero-bias operation became possible which allowed the study of the defects without moving them by the electric field. Yokoyama et al. (1999), in the same group, imaged Ag on $\mathrm{Si}$ by FM-AFM. In Fig. 38, the $\alpha-\mathrm{Al}_{2} \mathrm{O}_{3}$ surface in its $\sqrt{31} \times \sqrt{31} R+9^{\circ}$ high-temperature reconstruction is imaged by FM-AFM. These data demonstrate the use of FM-AFM for the surface science of insulators (see also Pethica and Egdell, 2001).

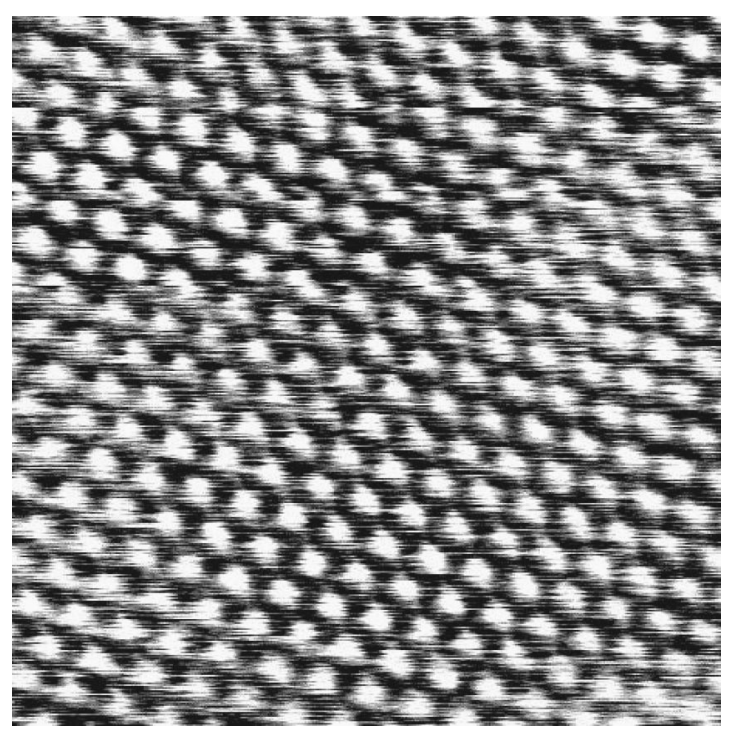

FIG. 36. FM-AFM image of a xenon thin film. Image size 70 $\times 70 \AA^{2}$. The maxima correspond to individual $\mathrm{Xe}$ atoms. Sputtered Si-tip, $f_{0}=160 \mathrm{kHz}, \Delta f=-92 \mathrm{~Hz}, A=9.4 \mathrm{~nm}, T$ $=22 \mathrm{~K}$, approximately $20 \mathrm{pm}$ corrugation. From Allers et al., 1999b.

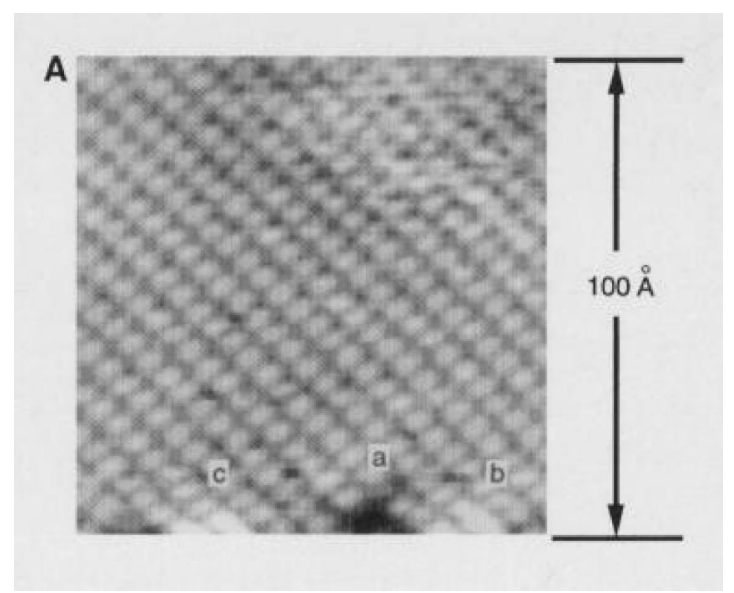

FIG. 37. Noncontact ultrahigh-vacuum AFM image of the cleaved InP(110) surface. The scan area was $100 \AA$ by $100 \AA$. Experimental conditions: spring constant of the cantilever $k$ $=34 \mathrm{~N} / \mathrm{m}$, mechanical resonant frequency $\nu_{0}=151 \mathrm{kHz}$, vibration amplitude $A=20 \mathrm{~nm}$, and frequency shift $\Delta \nu=-6 \mathrm{~Hz}$. Atomic defects (a) and adsorbates [(b) and (c)] are visible. Reprinted from Sugawara et al., 1995. Copyright (1995) American Association for the Advancement of Science.

\section{B. Spectroscopy}

At room temperature, lateral and vertical thermal drift usually prevents the performance of spectroscopy experiments directly over a specific atom, and frequency-versus-distance measurements suffer from thermal drift (Giessibl, 1995). However, at low temperatures, it is possible to perform spectroscopic measurements (Allers et al., 2002). Hölscher, Schwarz, et al. (1999) have performed frequency-versus-distance mea-

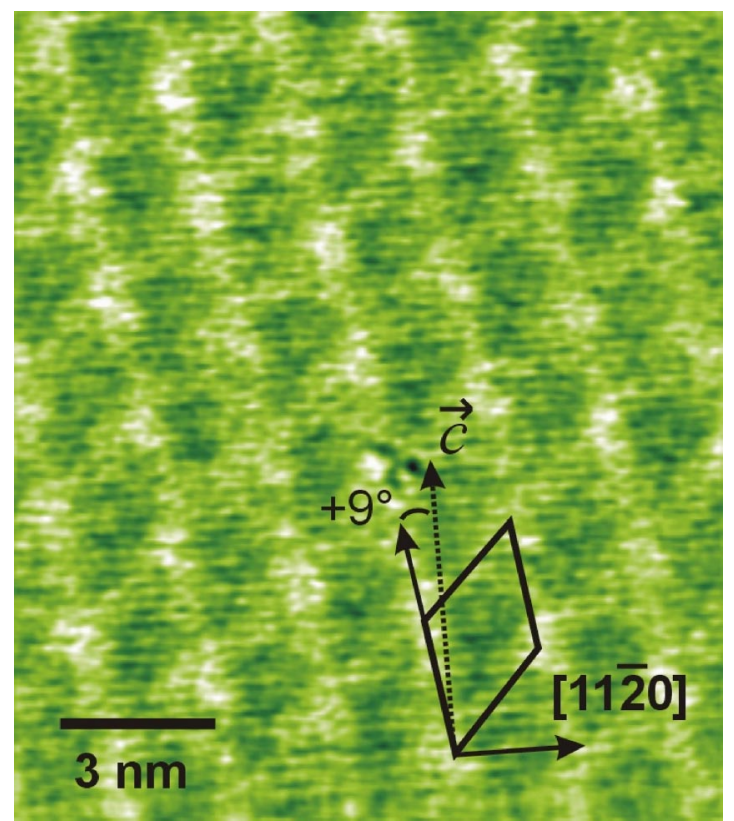

FIG. 38. (Color in online edition) FM-AFM image of an $\mathrm{Al}_{2} \mathrm{O}_{3}$ surface. Si-tip, $f_{0}=75 \mathrm{kHz}, \Delta f=-92 \mathrm{~Hz}, k=3 \mathrm{~N} / \mathrm{m}, A$ $=76 \mathrm{~nm}$, ambient temperature. From Barth and Reichling, 2001. Reprinted with permission by Nature (London). 


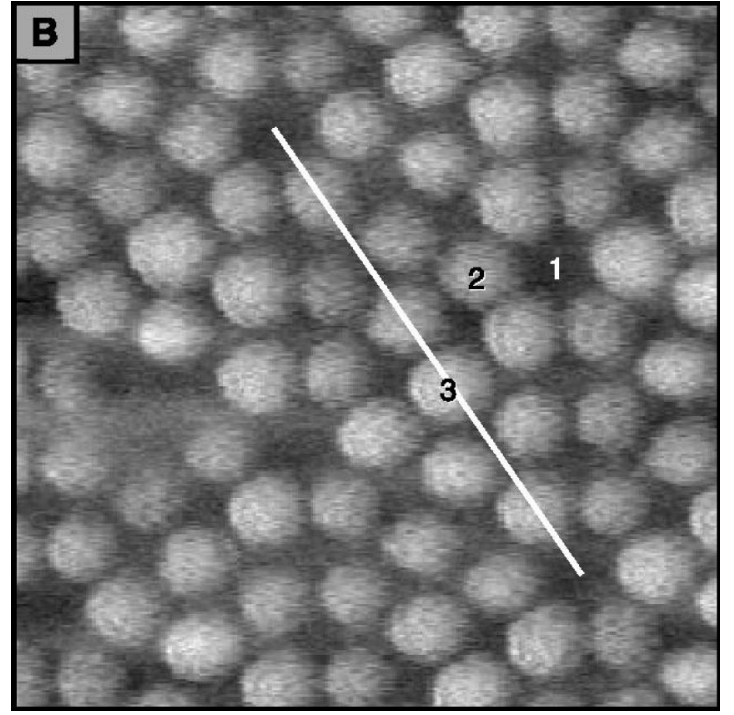

FIG. $39.6 \mathrm{~nm} \times 6 \mathrm{~nm}$ constant-frequency-shift image $(\Delta f$ $=-38 \mathrm{~Hz}$, rms error $1.15 \mathrm{~Hz}$, scan speed $2 \mathrm{~nm} / \mathrm{s}$ ). The labels 1 , 2 , and 3 indicate the positions of frequency-distance measurements in Fig. 40. Reprinted from Lantz et al., 2001. Copyright (2001) American Association for the Advancement of Science.

surements with a silicon cantilever and a graphite sample with several different amplitudes and used their deconvolution algorithm to calculate the tip-sample potential.

In 2001, Lantz et al. (2001) performed spectroscopy over specific atomic sites on the silicon surface. Figure 39 shows three distinct sites in the silicon $7 \times 7$ unit cell, where frequency-shift data were collected. Figure 40 shows the corresponding frequency-shift data and the corresponding forces, calculated with the algorithm proposed by Dürig (1999b). This is a significant breakthrough, because the measured forces are mainly caused by the interaction of two single atoms (see also the perspective by de Lozanne, 2001).

\section{NEW DEVELOPMENTS}

\section{A. Dissipation measurements and theory}

As early as 1991, Denk and Pohl (1991) used FMAFM to record the drive signal required to maintain a constant cantilever amplitude. In the distance regime covered by their early experiment, they found that the major dissipation mechanism is due to Ohmic losses of currents that are induced by the variable capacitance (due to oscillation) of the tip-sample assembly in connection with a constant tip-sample bias voltage. They obtained dissipation images on semiconductor heterostructures with a feature size of some $10 \mathrm{~nm}$ and coined the term "scanning dissipation microscopy." Lüthi et al. (1997) recorded the damping signal in atomic resolution experiments on silicon. Today, a number of theories have been proposed to explain the energy loss in dynamic atomic force microscopy (Dürig, 1999a; Gauthier and Tsukada, 1999; Sasaki and Tsukada, 2000; Kantorov-
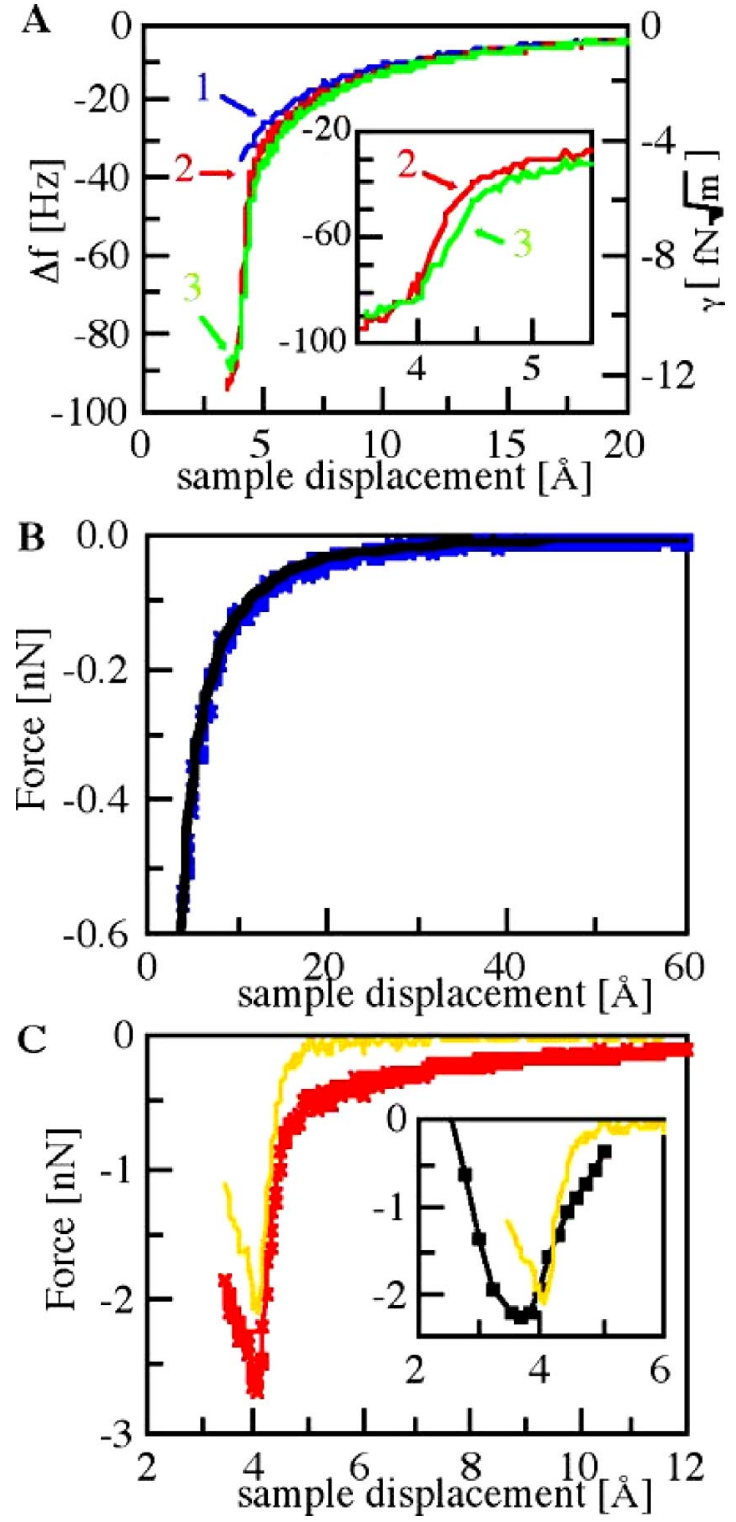

FIG. 40. (Color in online edition) Frequency-shift versus distance data measured above the positions labeled 1, 2, and 3 in Fig. 39. Reprinted from Lantz et al., 2001. Copyright (2001) American Association for the Advancement of Science.

ich, 2001). Recently, dissipative lateral forces have also been studied; see Pfeiffer et al. (2002) and below.

\section{B. Off-resonance technique with small amplitudes}

Pethica early identified the problem of the long-range background forces and searched for a way to minimize them (AFM challenge number 3, Sec. III). In dynamic force microscopy, the contribution of various force components $F_{i}$ with a corresponding range $\lambda_{i}$ to the imaging signal is a function of the cantilever oscillation amplitude $A$. For $A \gg \lambda$, the imaging signal is proportional to $\Sigma_{i} F_{i} \sqrt{\lambda_{i}}$, while for $A \ll \lambda$, the imaging signal is proportional to $\Sigma_{i} F_{i} / \lambda_{i}$ (see Sec. VII.A). Thus, for small amplitudes, the imaging signal is proportional to the force gradient and the weight of short-range forces is much 
larger than the weight of long-range forces. This fact has been used in an off-resonance technique developed by Hoffmann, Oral, Grimble, Özer, Jeffrey, and Pethica (2001). In this technique, a tungsten cantilever with $k$ $\approx 300 \mathrm{~N} / \mathrm{m}$ is oscillated at a frequency far below its resonance frequency with an amplitude $A_{0}$ of the order of $0.5 \AA$. When the cantilever comes close to the sample, the oscillation amplitude changes according to

$$
A=\frac{A_{0}}{1+k_{t s} / k}
$$

with a tip-sample stiffness $k_{t s}$. Two other AFM challenges, namely, the instability problem and the $1 / f$ noise problem, are also solved because of the stiff cantilever and the dynamic mode. Conceptually, this smallamplitude off-resonance technique is very attractive due to its simplicity in implementation and interpretation. A lock-in technique can be used to measure $A$, which improves the signal-to-noise ratio. The quality of the images presented is so far not as good as the quality of classic or small-amplitude FM-AFM data, possibly because the scanning speed is slow and thermal drift is a problem. Ongoing work has to show whether the image quality issues are due merely to technical imperfections or to more fundamental reasons. Atomic dissipation processes (Hoffmann, Jeffery, et al., 2001) and forceversus-distance data (Oral et al., 2001) have been measured with the technique.

\section{Dynamic mode with stiff cantilevers and small amplitudes}

Intuitively, the amplitudes that are used in classic FMAFM are much too large: if a silicon atom were magnified to the size of an orange, the average distance of the cantilever used in classic noncontact AFM mode would amount to $15 \mathrm{~m}$. The necessity of such large amplitudes was outlined in Sec. III. Intuitively, it was clear that greater sensitivity to short-range forces should be achieved with small amplitudes. It was even planned to use the thermal amplitude (Giessibl, 1994) to enhance short-range force contributions. However, empirical findings showed that because of the stability issues outlined above, large amplitudes had to be used with the relatively soft cantilevers that were available. Similar detours were taken in the development of the STM in several respects - the first STM's were insulated from external vibrations by levitation on superconducting magnets, and the first STM tips were fabricated using complicated mechanical and chemical preparation techniques, while later STM's used much simpler systems for vibration insulation and tip preparation (see Binnig, 1997, p. 59).

In FM-AFM, it was finally shown that small amplitudes do work, but only if extremely stiff cantilevers are used. After the theoretical proof of the benefits of using small amplitudes with stiff cantilevers (Giessibl et al., 1999), we tried to convince manufacturers of piezoresistive cantilevers to make devices with $k \approx 500$ $\mathrm{N} / \mathrm{m}$ - without success. However, the theoretical findings gave us enough confidence to modify a quartz tuning

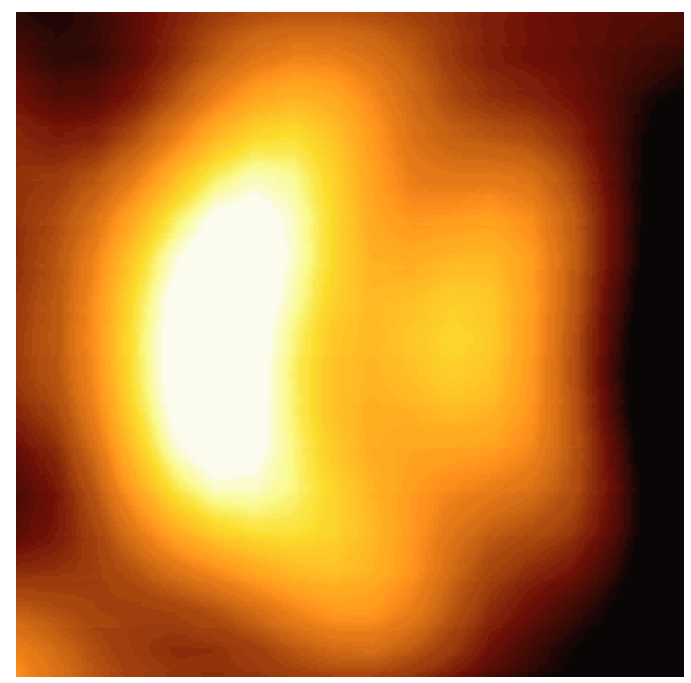

FIG. 41. (Color in online edition) Image of a single adatom on $\mathrm{Si}(111)-(7 \times 7)$. A $3 s p^{3}$ state points towards the surface normal on the Si (111) surface, and the image of this atom should be symmetric with respect to the $z$ axis. Because images in an AFM are a convolution of tip and sample states, and the sample state is well known in this case, the tip state is most likely to be two $3 s p^{3}$ states originating in a single Si tip atom (see Giessibl, Hembacher et al., 2000). Image size: 6.6 $\times 6.6 \AA^{2}$ lateral, $1.4 \AA$ vertical.

fork to a quartz cantilever sensor with a stiffness of roughly $2 \mathrm{kN} / \mathrm{m}$ (the qPlus sensor; see Fig. 11 and Giessibl, 1996, 1998). Even the first experiments were successful, yielding AFM images of silicon with excellent resolution. For the first time, clear features within the image of a single atom were observed (see Fig. 41). The structure of these images was interpreted as originating from the orbitals of the tip atom, the first observation of

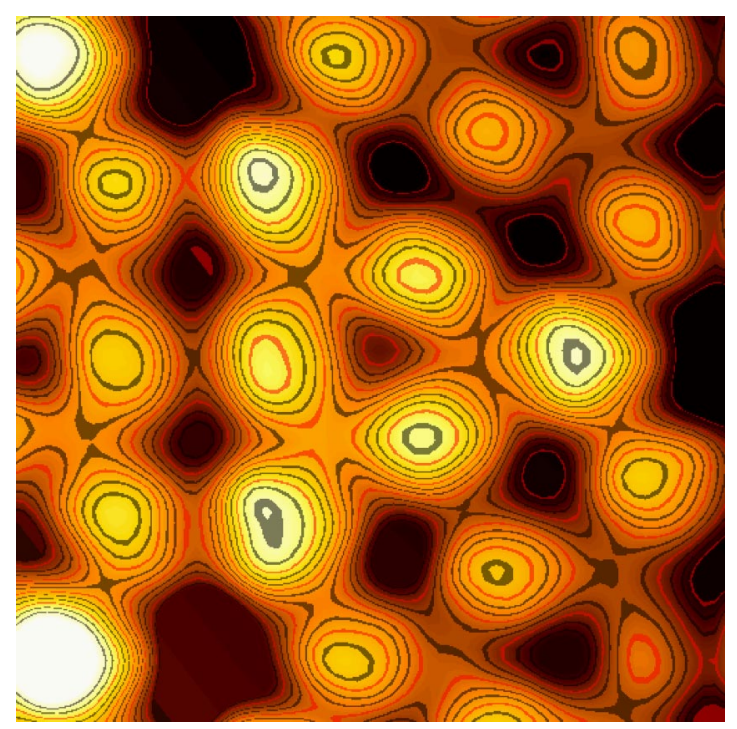

FIG. 42. (Color in online edition) Image of a $\mathrm{Si}(111)-(7 \times 7)$ surface imaged with a qPlus sensor. Parameters: $k=1800$ $\mathrm{N} / \mathrm{m}, A=2.5 \AA, f_{0}=14772 \mathrm{~Hz}, \Delta f=+4 \mathrm{~Hz}, \gamma=28 \mathrm{fN} \sqrt{\mathrm{m}}$. Image size: $40 \AA$ lateral, $1.4 \AA$ vertical. Source: Giessibl, Bielefeldt, et al., 2001. 


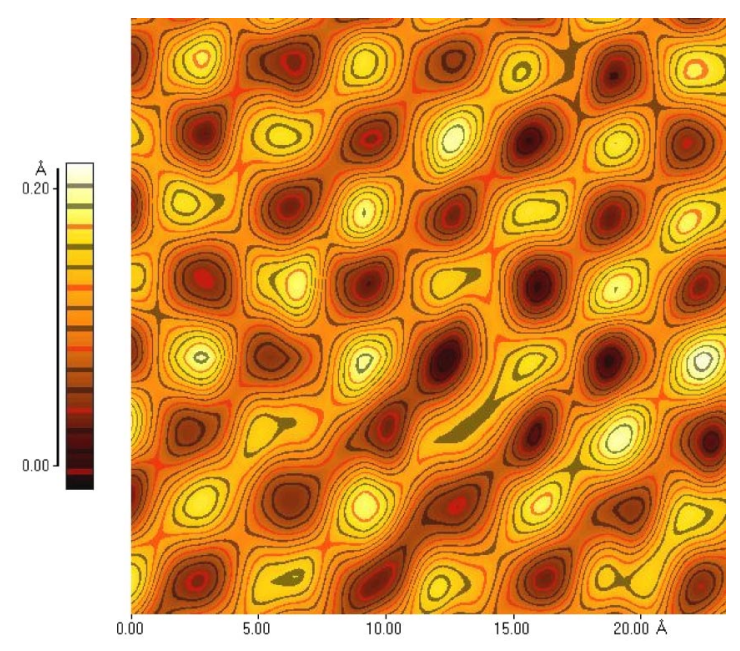

FIG. 43. (Color in online edition) Image of a $\mathrm{KCl}(001)$ surface imaged with a qPlus sensor. Parameters: $k=1800 \mathrm{~N} / \mathrm{m}, A$ $=9 \AA, f_{0}=14772 \mathrm{~Hz}, \Delta f=-4 \mathrm{~Hz}, \gamma=-13 \mathrm{fN} \sqrt{\mathrm{m}}$. Image size: $23.5 \AA$ lateral, $0.3 \AA$ vertical. The contour lines are spaced vertically by approximately $3 \mathrm{pm}$.

charge structure within atoms in real space (Giessibl, Hembacher, et al., 2000). Figure 41 is an image of a single silicon adatom. Silicon adatoms display a single $s p^{3}$ dangling bond sticking out perpendicular from the surface. Thus the image of this atom is expected to be spherically symmetric with respect to the vertical axis. We interpret the image as being caused by an overlap of two $s p^{3}$ dangling bonds from the tip with the single dangling bond from the surface; for a detailed description see Giessibl, Hembacher, et al. (2000); Giessibl, Bielefeldt, et al. (2001). On the subatomic level, the image is sensitive to the chemical identity and the structural surroundings of the front atom of the tip. First attempts to engineer tips with a known symmetry are under way (Giessibl, Hembacher, et al., 2001b). Figure 42 is an image of a $\operatorname{Si}(111)-(7 \times 7)$ surface imaged with a qPlus sensor with a [111]-oriented Si tip (see Fig. 11) with extremely small amplitudes $(2.5 \AA)$ and even positive frequency shifts, i.e., repulsive forces. The tip was found to be extremely stable compared to [001]-oriented tips.

It should be noted that the claim of subatomic resolution capability is under debate. Hug et al. (2001) pro- posed that the experimental observations of subatomic resolution could be artifacts due to feedback errors. However, Giessibl, Hembacher, et al. (2001a) concluded that analysis of the feedback signals rules out feedback artifacts. So far, subatomic resolution has not been reported using classic noncontact AFM. The smallamplitude technique with very stiff cantilevers allows the achievement of tip-sample distances close to the bulk distances and obtains single-atom images with nontrivial internal structures (subatomic resolution) on silicon (Giessibl, Bielefeldt, et al., 2001) and rare-earth metal atoms (Herz et al., 2003). The enhanced resolution of short-range forces as a result of using small amplitudes was confirmed experimentally by Eguchi and Hasegawa (2002). Furthermore, Huang, Cuma, and Liu (2003) have performed $a b$ initio calculations and confirmed that atomic substructures linked to atomic orbitals are expected to occur when tip and sample reach distances similar to bulk next neighbor spacings.

The capacity of the stiff-cantilever small-amplitude technique to image standard insulators with moderate short-range forces is shown in Fig. 43, where a $\mathrm{KCl}$ [001] surface is imaged with a qPlus sensor with a silicon tip.

\section{Dynamic lateral force microscopy}

Experiments on atomic friction became possible with the invention of the lateral force microscope, introduced in 1987 by Mate et al. (1987). The resolution power of the lateral force microscope has been improving steadily, opening many applications, including high-resolution wear studies on $\mathrm{KBr}$ (Gnecco et al., 2002). However, the observation of single atomic defects has not been achieved by quasistatic lateral force microscopy. Because of the similarity of the challenges faced by normal force and lateral force microscopy, the frequencymodulation method has been tried. Pfeiffer et al. (2002) imaged atomic steps with this technique, and recently Giessibl et al. (2002) achieved true atomic resolution with a large-stiffness, small-amplitude lateral frequencymodulated AFM (see Fig. 12). In addition to the frequency shift, the dissipated power between tip and sample was measured as the difference between the power required for maintaining a constant amplitude when the cantilever is close to the sample and the power
$\mathbf{A}$

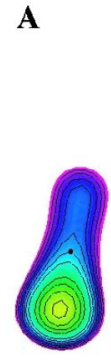

$4 \AA$
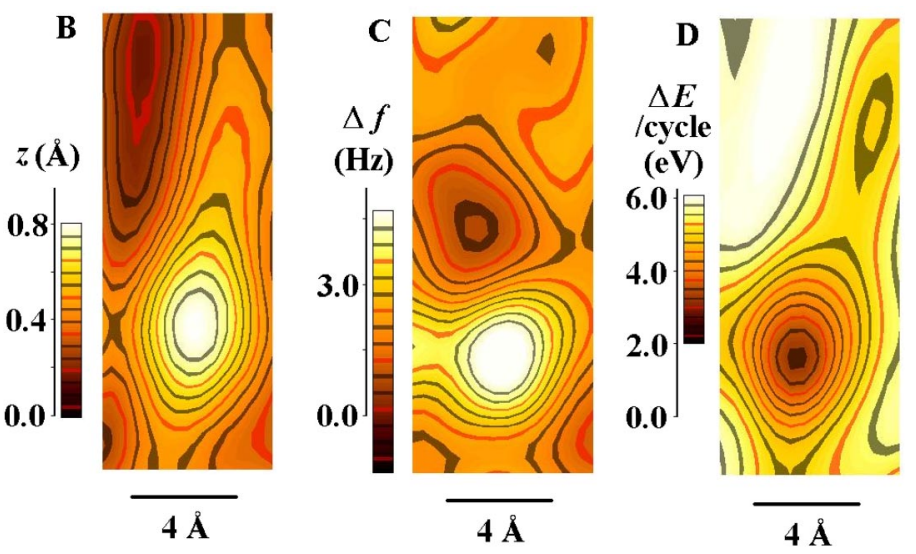

FIG. 44. (Color in online edition) Lateral force microscopy data on a single adatom of $\mathrm{Si}(111)$ imaged with a qPlus lateral sensor: (A) Simulated constant average current topographic image; (B) experimental topographic image of a single adatom; (C) experimental data of frequency shift; (D) experimental data of dissipation energy. 
required when the cantilever is far away from the sample, yielding a connection to friction forces. Figure 44 shows experimental data on the conservative and dissipative force components between a single adatom on a Si surface and a single atom tip. When the cantilever is vibrating laterally directly over an adatom, almost no extra dissipation occurs, while when approaching and retracting the tip from the side, a dissipation of the order of a few eV per oscillation cycle is measured. The data are interpreted with a theory going back to Tomlinson (1929).

\section{SUMMARY AND CONCLUSIONS}

Imaging flat surfaces with atomic resolution in direct space-regardless of their electrical conductivity-is standard practice now thanks to atomic force microscopy. The theoretical understanding of AFM has been advanced considerably and a direct link between the experimental observables (frequency shift, damping, and average tunneling current) and the underlying physical concepts (conservative and dissipative forces) has been established. Forces can be deconvoluted from frequency-shift data easily and low-temperature spectroscopy experiments show an outstanding agreement between theoretical and experimental tip-sample forces. Atomic force microscopy yields information about the strength and geometry of chemical bonds between single atoms.

Four techniques for atomic-resolution force microscopy in vacuum are in use today: the classic frequencymodulation technique with large amplitudes and soft cantilevers (used by most experimenters); frequency modulation with small amplitudes and stiff cantilevers; the off-resonance technique originated by the Pethica group; and the amplitude-modulation method. The future will show if one of these techniques will survive as an optimal method or if all or some techniques will remain in use. Lateral force microscopy with true atomic resolution has been demonstrated using the smallamplitude/stiff-cantilever technique.

\section{OUTLOOK}

While FM atomic force microscopy is an established experimental technique, applications in the surface science of insulators are just starting to emerge. Atomic force microscopy is still more complicated than scanning tunneling microscopy, and these complications appear to deter many scientists. However, significant progress has been made in recent years, and exciting results can be expected in the surface science of insulators, where the AFM is a unique tool for atomic studies in direct space. The possibilities of atomic-resolution force microscopy are overwhelming: access to the very scaffolding of matter, the chemical bond. Progress in our physical understanding and subsequent simplification of the use and interpretation of AFM's has been significant over the past few years. Atomic force microscopy offers additional observables-forces and damping - that are even vectors with three spatial components-the tunneling current in an STM is a scalar entity. There are strong indications that dynamic force microscopy allows the imaging of features within single-atom images attributed to atomic valence orbitals. Thus the whole field of classic STM studies could be revisited with the enhanced resolution technique.

One of the greatest challenges of the AFM is the preparation of well-defined tips. As in the early days of the STM, tip preparation has acquired a certain mystique, with recipes ranging from sputtering to controlled collisions. Because the tip is closer to the sample in the AFM than in the STM, the stability of the front atom is more important in the AFM. Also, the chemical identity and backbond geometry of the tip is crucial in an AFM. The use of nanotubes as tips appeared to be promising, but, the observations expressed in Fig. 4 of Binnig and Rohrer (1987) regarding the necessity of a rigid, coneshaped tip are to be heeded, especially in the AFM, where forces between tip and sample are larger than in the STM.

Atomic manipulation by scanning probe microscopes is an exciting challenge. Arranging atoms on conducting surfaces has been possible by use of the STM for a decade (Eigler and Schweizer, 1990; Crommie et al., 1993; Meyer et al., 1996; Kliewer et al., 2000; Manoharan et al., 2000). Morita and Sugawara (2002) have successfully extracted single atoms from a $\mathrm{Si}(111)-(7 \times 7)$ surface in a controlled fashion with a force microscope. An exciting extension of this work would be possible if atoms could also be deposited with atomic precision by atomic force microscopy. The construction of one-dimensional conductors or semiconductors on insulating substrates would allow the building of electronic circuits consisting only of a few atoms. While it appears difficult to manipulate atoms with a probe that oscillates to and from the surface with amplitudes of the order of $10 \mathrm{~nm}$, the continuous decrease in amplitudes used in dynamic force microscopy might allow us someday soon to move atoms in a controlled manner by atomic force microscopy.

\section{ACKNOWLEDGMENTS}

The author thanks Jochen Mannhart for his longtime collaboration, enthusiastic support, and many discussions. Special thanks to all current and former colleagues in Augsburg for their contributions: $\mathrm{H}$. Bielefeldt, Ph. Feldpausch, S. Hembacher, A. Herrnberger, M. Herz, U. Mair, Th. Ottenthal, Ch. Schiller, and K. Wiedenmann. Many thanks to G. Binnig, Ch. Gerber, and C. F. Quate, who have contributed in many ways to the author's work on the atomic force microscope. The author is indebted to the following colleagues for supplying figures and for discussions: T. Akitoshi, T. Albrecht, W. Allers, A. Baratoff, C. Barth, G. Binnig, U. Dürig, R. Erlandsson, Ch. Gerber, H.-J. Güntherodt, L. Howald, H. Hug, M. Lantz, R.B. Marcus, E. Meyer, O. Ohlsson, T. Oosterkamp, C. F. Quate, M. Reichling, A. Schwarz, U. D. Schwarz, M. Tortonese, 
and R. Wiesendanger. L. Howald and D. Brändlin from Nanosurf AG have made crucial contributions to our project by providing prototypes and final versions of their frequency-to-voltage converters and fruitful discussions about FM-AFM. Special thanks to G. Binnig, K. Dransfeld, U. Dürig, S. Fain, S. Hembacher, and J. Mannhart for editorial suggestions. This work was supported by the Bundesministerium für Bildung und Forschung (Project No. 13N6918).

\section{REFERENCES}

Abdurixit, A., A. Baratoff, and E. Meyer, 1999, "Molecular dynamics simulations of dynamic force microscopy: application to the Si(111)-7×7 surface," Appl. Surf. Sci. 157, 355360.

Abramowitz, M., and I. Stegun, 1970, Eds., Handbook of Mathematical Functions, 9th ed. (Dover, New York).

Akamine, S., R. C. Barrett, and C. F. Quate, 1990, "Improved atomic force microscopy images using cantilevers with sharp tips," Appl. Phys. Lett. 57, 316-318.

Albrecht, T. R., 2000, private communication.

Albrecht, T. R., S. Akamine, T. E. Carver, and C. F. Quate, 1990, "Microfabrication of cantilever styli for the atomic force microscope," J. Vac. Sci. Technol. A 8, 3386-3396.

Albrecht, T. R., P. Grutter, H. K. Horne, and D. Rugar, 1991, "Frequency modulation detection using high-Q cantilevers for enhanced force microscope sensitivity," J. Appl. Phys. 69, 668-673.

Allers, W., A. Schwarz, and U. D. Schwarz, 2002, in Noncontact Atomic Force Microscopy, edited by S. Morita, R. Wiesendanger, and E. Meyer (Springer, Berlin), Chap. 14, pp. 233-256.

Allers, W., A. Schwarz, U. D. Schwarz, and R. Wiesendanger, 1999a, "Dynamic scanning force microscopy at low temperatures on a van der Waals surface: graphite (0001)," Appl. Surf. Sci. 140, 247-252.

Allers, W., U. D. Schwarz, A. Schwarz, and R. Wiesendanger, 1998, "A scanning force microscope with atomic resolution in ultrahigh vacuum and at low temperatures," Rev. Sci. Instrum. 69, 221-225.

Allers, W., U. D. Schwarz, A. Schwarz, and R. Wiesendanger, 1999b, "Dynamic scanning force microscopy at low temperatures on a noble-gas crystal: Atomic resolution on the xenon [111] surface," Europhys. Lett. 48, 276-279.

Anczykowski, B., B. Gotsmann, H. Fuchs, J. P. Cleveland, and V. B. Elings, 1999, "How to measure energy dissipation in dynamic mode atomic force microscopy," Appl. Surf. Sci. 140, 376-382.

Anczykowski, B., D. Krüger, and H. Fuchs, 1996, "Cantilever dynamics in quasinoncontact force microscopy: Spectroscopic aspects," Phys. Rev. B 53, 15 485-15 488.

Arai, T., and M. Tomitori, 2002, in Noncontact Atomic Force Microscopy, edited by S. Morita, R. Wiesendanger, and E. Meyer (Springer, Berlin), Chap. 4, pp. 79-92.

Ashcroft, N. W., and N. D. Mermin, 1981, Solid State Physics (Saunders College, Philadelphia).

Bammerlin, M., R. Lüthi, E. Meyer, A. Baratoff, J. Lü, M. Guggisberg, C. Gerber, L. Howald, and H. J. Güntherodt, 1997, "True atomic resolution on the surface of an insulator via ultrahigh vacuum dynamic force microscopy," Probe Microsc. 1, 3-9.
Baratoff, A., 1997, "Perturbation theory of large-amplitude dynamic force microscopy," talk at First International Workshop on Noncontact-Atomic Force Microscopy, Osaka, Japan (unpublished).

Barth, C., and M. Reichling, 2001, "Imaging the atomic arrangements on the high-temperature reconstructed $\alpha-\mathrm{Al}_{2} \mathrm{O}_{3}(0001)$ surface," Nature (London) 414, 54-56.

Barth, C., and M. Reichling, 2002, in Noncontact Atomic Force Microscopy, edited by S. Morita, R. Wiesendanger, and E. Meyer (Springer, Berlin), Chap. 8, 135-146.

Bartzke, K., T. Antrack, K.-H. Schmidt, E. Dammann, and C. Schatterny, 1993, "The needle sensor-a micromechanical detector for atomic force microscopy," Int. J. Optoelectron. 8, 669-676.

Baym, G., 1969, Lectures on Quantum Mechanics (Benjamin, New York).

Bazant, M. Z., and E. Kaxiras, 1997, "Environment-dependent interatomic potential for bulk silicon," Phys. Rev. B 56, 8542 8552.

Becker, R., 1969, Theorie der Wärme, 3rd ed. (Springer, Berlin).

Bennewitz, R., M. Bammerlin, and E. Meyer, 2002, in Noncontact Atomic Force Microscopy, edited by S. Morita, R. Wiesendanger, and E. Meyer (Springer, Berlin), Chap. 5, pp. 93108.

Bennewitz, R., C. Gerber, and E. Meyer, 2000, in "Proceedings of the Second International Workshop on Noncontact Atomic Force Microscopy, Pontresina, Switzerland, September 1-4, 1999," Appl. Surf. Sci. 157, 207-428.

Bennewitz, R., O. Pfeiffer, S. Schär, V. Barwich, E. Meyer, and L. Kantorovich, 2002, "Atomic corrugation in nc-AFM of alkali halides,” Appl. Surf. Sci. 188, 232-237.

Bielefeldt, H., and F. J. Giessibl, 1999, "A simplified but intuitive analytical model for intermittent-contact mode force microscopy based on Hertzian mechanics," Surf. Sci. 440, L863-L867.

Binnig, G., 1986, “Atomic Force Microscope and Method for Imaging Surfaces with Atomic Resolution," US Patent No. 4,724,318.

Binnig, G., 1992, "Force microscopy," Ultramicroscopy 42-44, 7-15.

Binnig, G., 1997, Aus dem Nichts, 2nd ed. (Piper, München).

Binnig, G., C. F. Quate, and C. Gerber, 1986, "Atomic force microscope," Phys. Rev. Lett. 56, 930-933.

Binnig, G., and H. Rohrer, 1985, "The scanning tunneling microscope," Sci. Am. 253 (2), 40-46.

Binnig, G., and H. Rohrer, 1987, "Nobel Lecture: Scanning tunneling microscopy-from birth to adolescence," Rev. Mod. Phys. 59, 615-625.

Binnig, G., and H. Rohrer, 1999, "In touch with atoms," Rev. Mod. Phys. 71, S324-S330.

Binnig, G., H. Rohrer, C. Gerber, and E. Weibel, 1982, "Surface studies by scanning tunneling microscopy," Phys. Rev. Lett. 49, 57-61.

Binnig, G., H. Rohrer, C. Gerber, and E. Weibel, 1983, “7×7 reconstruction on $\mathrm{Si}(111)$ resolved in real space," Phys. Rev. Lett. 50, 120-123.

Burnham, N., and R. J. Colton, 1989, "Measuring the nanomechanical and surface forces of materials using an atomic force microscope," J. Vac. Sci. Technol. A 7, 2906-2913.

Chen, C. J., 1993, Introduction to Scanning Tunneling Microscopy (Oxford University Press, New York). 
Ciraci, S., A. Baratoff, and I. P. Batra, 1990, "Tip-sample interaction effects in scanning-tunneling and atomic-force microscopy," Phys. Rev. B 41, 2763-2775.

Coulson, C. A., and R. McWeeny, 1991, Coulson's Valence (Oxford University Press, New York).

Crommie, M. F., C. P. Lutz, and D. M. Eigler, 1993, "Confinement of electrons to quantum corrals on a metal surface," Science 262, 218-220.

de Lozanne, A., 2001, "Atomic force microscopy: You may squeeze the atoms but don't mangle the surface!" Science 291, 2561-2562.

Denk, W., and D. W. Pohl, 1991, "Local electrical dissipation imaged by scanning force microscopy," Appl. Phys. Lett. 59, 2171-2173.

Drakova, D., 2001, “Theoretical modelling of scanning tunneling microscopy, scanning tunneling spectroscopy and atomic force microscopy," Rep. Prog. Phys. 64, 205-290.

Dürig, U., 1999a, "Conservative and dissipative interactions in dynamic force microscopy," Surf. Interface Anal. 27, 467473.

Dürig, U., 1999b, "Relations between interaction force and frequency shift in large-amplitude dynamic force microscopy," Appl. Phys. Lett. 75, 433-435.

Dürig, U., 2000, "Interaction sensing in dynamic force microscopy," New J. Phys. 2, 5.1-5.12.

Dürig, U., J. K. Gimzewski, and D. W. Pohl, 1986, "Experimental observation of forces acting during scanning tunneling microscopy," Phys. Rev. Lett. 57, 2403-2406.

Dürig, U., H. P. Steinauer, and N. Blanc, 1997, "Dynamic force microscopy by means of the phase-controlled oscillator method," J. Appl. Phys. 82, 3641-3651.

Dürig, U., O. Züger, and D. W. Pohl, 1990, "Observation of metallic adhesion using the scanning tunneling microscope," Phys. Rev. Lett. 65, 349-352.

Dürig, U., O. Züger, and A. Stalder, 1992, "Interaction force detection in scanning probe microscopy: Methods and applications," J. Appl. Phys. 72, 1778-1798.

Edwards, H., L. Taylor, and W. Duncan, 1997, "Fast, high resolution atomic force microscopy using a quartz tuning fork as actuator and sensor," J. Appl. Phys. 82, 980-984.

Eguchi, T., and Y. Hasegawa, 2002, "High-resolution atomic force microscopic imaging of the $\operatorname{Si}(111)(7 \times 7)$ surface: Contribution of short-range force to the images," Phys. Rev. Lett. 89, 266105.

Eigler, D. M., and E. K. Schweizer, 1990, "Positioning single atoms with a scanning tunnelling microscope," Nature (London) 344, 524-526.

Erlandsson, R., L. Olsson, and P. Martensson, 1997, "Inequivalent atoms and imaging mechanisms in ac-mode atomic-force microscopy of Si(111) $(7 \times 7)$," Phys. Rev. B 54, R8309R8312.

Foster, A., A. Shluger, C. Barth, and M. Reichling, 2002, in Noncontact Atomic Force Microscopy, edited by S. Morita, R. Wiesendanger, and E. Meyer (Springer, Berlin), Chap. 17, pp. 305-348.

French, R. H., 2000, "Origins and applications of London dispersion forces and Hamaker constants in ceramics," J. Am. Ceram. Soc. 83, 2117-2146.

Fukui, K., and Y. Iwasawa, 2002, in Noncontact Atomic Force Microscopy, edited by S. Morita, R. Wiesendanger, and E. Meyer (Springer, Berlin), Chap. 10, pp. 167-182.
Fukui, K., H. Onishi, and Y. Iwasawa, 1997, “Atom-resolved image of the $\mathrm{TiO}_{2}(110)$ surface by noncontact atomic force microscopy,” Phys. Rev. Lett. 79, 4202-4205.

Garcia, R., and R. Perez, 2002, "Dynamic atomic force microscopy methods," Surf. Sci. Rep. 47, 197-301.

Gauthier, M., L. Kantorovich, and M. Tsukada, 2002, in Noncontact Atomic Force Microscopy, edited by S. Morita, R. Wiesendanger, and E. Meyer (Springer, Berlin), Chap. 19, pp. 371-394.

Gauthier, M., R. Perez, T. Arai, M. Tomitori, and M. Tsukada, 2002, "Interplay between nonlinearity, scan speed, damping, and electronics in frequency modulation atomic-force microscopy,” Phys. Rev. Lett. 89, 146104.

Gauthier, M., N. Sasaki, and M. Tsukada, 2001, "Dynamics of the cantilever in noncontact dynamic force microscopy: The steady-state approximation and beyond," Phys. Rev. B 64, 085409.

Gauthier, M., and M. Tsukada, 1999, "Theory of noncontact dissipation force microscopy," Phys. Rev. B 60, $11716-$ 11722.

Giessibl, F. J., 1991, Rastertunnel- und Rasterkraftmikroskopie bei 4,2 K im Ultrahochvakuum, Ph.D. thesis (LudwigMaximilians-Universität, München, Germany).

Giessibl, F. J., 1992, "Theory for an electrostatic imaging mechanism allowing atomic resolution of ionic crystals by atomic force microscopy," Phys. Rev. B 45, 13 815-13 818.

Giessibl, F. J., 1994, "Atomic force microscopy in ultrahigh vacuum,” Jpn. J. Appl. Phys., Part 1 33, 3726-3734.

Giessibl, F. J., 1995, "Atomic resolution of the silicon (111)- $(7 \times 7)$ surface by atomic force microscopy," Science 267, 68-71.

Giessibl, F. J., 1996, "Vorrichtung zum berührungslosen Abtasten einer Oberfläche und Verfahren dafür," Offenlegungsschrift German Patent Office DE 19633546.

Giessibl, F. J., 1997, "Forces and frequency shifts in atomic resolution dynamic force microscopy," Phys. Rev. B 56, $16010-16015$.

Giessibl, F. J., 1998, "High-speed force sensor for force microscopy and profilometry utilizing a quartz tuning fork," Appl. Phys. Lett. 73, 3956-3958.

Giessibl, F. J., 2000, “Atomic resolution on $\mathrm{Si}(111)-(7 \times 7)$ by noncontact atomic force microscopy with a force sensor based on a quartz tuning fork," Appl. Phys. Lett. 76, 14701472.

Giessibl, F. J., 2001, “A direct method to calculate tip-sample forces from frequency shifts in frequency-modulation atomic force microscopy," Appl. Phys. Lett. 78, 123-125.

Giessibl, F. J., 2002, in Noncontact Atomic Force Microscopy, edited by S. Morita, R. Wiesendanger, and E. Meyer (Springer, Berlin), Chap. 2, pp. 11-46.

Giessibl, F. J., and H. Bielefeldt, 2000, "Physical interpretation of frequency-modulation atomic force microscopy," Phys. Rev. B 61, 9968-9971.

Giessibl, F. J., H. Bielefeldt, S. Hembacher, and J. Mannhart, 1999, "Calculation of the optimal imaging parameters for frequency modulation atomic force microscopy," Appl. Surf. Sci. 140, 352-357.

Giessibl, F. J., H. Bielefeldt, S. Hembacher, and J. Mannhart, 2001, "Imaging of atomic orbitals with the atomic force microscope-experiments and simulations," Ann. Phys. (Leipzig) 10, 887-910. 
Giessibl, F. J., and G. Binnig, 1992a, "Atomic resolution of potassium bromide (001) with an atomic force microscope and calculation of tip shape," unpublished.

Giessibl, F. J., and G. Binnig, 1992b, "True atomic resolution on $\mathrm{KBr}$ with a low-temperature atomic force microscope in ultrahigh vacuum," Ultramicroscopy 42-44, 281-286.

Giessibl, F., C. Gerber, and G. Binnig, 1991, "A lowtemperature atomic force/scanning tunneling microscope for ultrahigh vacuum," J. Vac. Sci. Technol. B 9, 984-988.

Giessibl, F. J., S. Hembacher, H. Bielefeldt, and J. Mannhart, 2000, "Subatomic features on the silicon (111)- $(7 \times 7)$ surface observed by atomic force microscopy," Science 289, 422425.

Giessibl, F. J., S. Hembacher, H. Bielefeldt, and J. Mannhart, 2001a, "Response to technical comment: Subatomic features in atomic force microscopy images," Science 291, 2509a.

Giessibl, F. J., S. Hembacher, H. Bielefeldt, and J. Mannhart, 2001b, "Imaging silicon with crystallographically oriented tips by atomic force microscopy," Appl. Phys. A: Mater. Sci. Process. 72, 15-17.

Giessibl, F. J., M. Herz, and J. Mannhart, 2002, "Friction traced to the single atom," Proc. Natl. Acad. Sci. U.S.A. 99, 12 006-12 010.

Giessibl, F. J., and M. Tortonese, 1997, "Self oscillating mode for frequency modulation non-contact atomic force microscopy," Appl. Phys. Lett. 70, 2529-2531.

Giessibl, F. J., and B. M. Trafas, 1994, "Piezoresistive cantilevers utilized for scanning tunneling and scanning force microscope in ultrahigh vacuum," Rev. Sci. Instrum. 65, 1923-1929. Gnecco, E., R. Bennewitz, and E. Meyer, 2002, “Abrasive wear on the atomic scale," Phys. Rev. Lett. 88, 215501.

Goldstein, H., 1980, Classical Mechanics (Addison-Wesley, Reading, MA).

Gotsmann, B., C. Schmidt, C. Seidel, and H. Fuchs, 1998, "Molecular resolution of an organic monolayer by dynamic AFM," Eur. Phys. J. B 4, 267-268.

Gotsmann, B., C. Schmidt, C. Seidel, and H. Fuchs, 1999, "Determination of tip-sample interaction forces from measured dynamic force spectroscopy curves," Appl. Surf. Sci. 140, 314-319.

Güntherodt, H.-J., and R. Wiesendanger, 1991, Eds., Scanning Tunneling Microscopy I-III (Springer, Berlin).

Güthner, P., 1992, Untersuchung der lokalen elektrischen Eigenschaften dünner ferroelektrischer Polymere, Ph.D. thesis (University of Konstanz) konstanzer Dissertationen Vol. 357.

Güthner, P., 1996, "Simultaneous imaging of $\mathrm{Si}(111) 7 \times 7$ with atomic resolution in scanning tunneling microscopy, atomic force microscopy, and atomic force microscopy noncontact mode," J. Vac. Sci. Technol. B 14, 2428-2431.

Güthner, P., U. C. Fischer, and K. Dransfeld, 1989, "Scanning near-field acoustic microscopy," Appl. Phys. B: Lasers Opt. 48, 89-92.

Hamaker, H. C., 1937, "The London-van der Waals attraction between spherical particles," Physica (Amsterdam) 4, 10581072.

Hartmann, U., 1991, "Theory of van der Waals microscopy," J. Vac. Sci. Technol. B 9, 465-469.

Hembacher, S., F. J. Giessibl, and J. Mannhart, 2002, "Evaluation of a force sensor based on a quartz tuning fork for operation at low temperatures and ultra-high vacuum," Appl. Surf. Sci. 188, 445-449.

Herz, M., F. J. Giessibl, and J. Mannhart, 2003, "Probing the shape of atoms in real space," Phys. Rev. B 68, 045301.
Hölscher, H., 2002, in Noncontact Atomic Force Microscopy, edited by S. Morita, R. Wiesendanger, and E. Meyer (Springer, Berlin), Chap. 18, pp. 349-370.

Hölscher, H., W. Allers, U. D. Schwarz, A. Schwarz, and R. Wiesendanger, 1999, "Calculation of the frequency shift in dynamic force microscopy," Appl. Surf. Sci. 140, 344-351.

Hölscher, H., A. Schwarz, W. Allers, U. D. Schwarz, and R. Wiesendanger, 2000, "Quantitative analysis of dynamic-forcespectroscopy data on graphite(0001) in the contact and noncontact regimes," Phys. Rev. B 61, 12 678-12 681.

Hölscher, H., U. D. Schwarz, and R. Wiesendanger, 1999, "Determination of tip-sample interaction potentials by dynamic force spectroscopy," Phys. Rev. Lett. 83, 4780-4783.

Hoffmann, P., 2003, in "Proceedings of the Fifth International Conference on Noncontact Atomic Force Microscopy, Montreal, Canada, August 12-14, 2002," Appl. Surf. Sci. (to be published).

Hoffmann, P. M., S. Jeffery, J. B. Pethica, H. Özgr Özer, and A. Oral, 2001, "Energy dissipation in atomic force microscopy and atomic loss processes," Phys. Rev. Lett. 87, 265502.

Hoffmann, P. M., A. Oral, R. A. Grimble, H. Özer, S. Jeffrey, and J. B. Pethica, 2001, "Direct measurement of interatomic force gradients using an ultra-low-amplitude atomic force microscope," Proc. R. Soc. London, Ser. A 457, 1161-1174.

Horowitz, P., and W. Hill, 1989, The Art of Electronics, 2nd ed. (Cambridge University Press, Cambridge/New York).

Hosoi, H., K. Sueoka, K. Hayakawa, and K. Mukasa, 2002, in Noncontact Atomic Force Microscopy, edited by S. Morita, R. Wiesendanger, and E. Meyer (Springer, Berlin), Chap. 7, pp. 125-134.

Howald, L., 1994, Rasterkraftmikroskopie an Silizium und Ionenkristallen in Ultrahochvakuum, Ph.D. thesis (Universität Basel, Switzerland).

Howald, L., R. Lüthi, E. Meyer, P. Güthner, and H.-J. Güntherodt, 1994, "Scanning force microscopy on the Si(111) $7 \times 7$ surface reconstruction," Z. Phys. B: Condens. Matter 93, 267-268.

Huang, M., M. Cuma, and F. Liu, 2003, "Seeing the atomic orbital: First-principles study of the effect of tip geometry on atomic force microscopy," Phys. Rev. Lett. 90, 256101.

Hug, H. J., and A. Baratoff, 2002, in Noncontact Atomic Force Microscopy, edited by S. Morita, R. Wiesendanger, and E. Meyer (Springer, Berlin), Chap. 20, pp. 395-432.

Hug, H. J., M. A. Lantz, A. Abdurixit, P. J. A. van Schendel, R. Hoffmann, P. Kappenberger, and A. Baratoff, 2001, "Technical Comment: Subatomic features in atomic force microscopy images," Science 291, 2509a.

Israelachvili, J., 1991, Intermolecular and Surface Forces, 2nd ed. (Academic, London).

Itoh, T., C. Lee, and T. Suga, 1996, "Deflection detection and feedback actuation using a self-excited piezoelectric $\mathrm{Pb}(\mathrm{Zr}, \mathrm{Ti}) \mathrm{O}_{3}$ microcantilever for dynamic scanning force microscopy," Appl. Phys. Lett. 69, 2036-2038.

Jarvis, M. R., R. Prez, and M. C. Payne, 2001, "Can atomic force microscopy achieve atomic resolution in contact mode?" Phys. Rev. Lett. 86, 1287-1290.

Jarvis, S. P., H. Tokumoto, and J. B. Pethica, 1997, "Measurement and interpretation of forces in the atomic force microscope," Probe Microsc. 1, 65-79.

Jarvis, S. P., H. Yamada, H. Tokumoto, and J. B. Pethica, 1996, "Direct mechanical measurement of interatomic potentials," Nature (London) 384, 247-249. 
Kantorovich, L. N., 2001, "A simple non-equilibrium theory of non-contact dissipation force microscopy,” J. Phys.: Condens. Matter 13, 945-958.

Karrai, K., and R. D. Grober, 1995, "Piezoelectric tip-sample distance control for near field optical microscopes," Appl. Phys. Lett. 66, 1842-1844.

Ke, S. H., T. Uda, I. Stich, and K. Terakura, 2001, "Firstprinciples simulation of atomic force microscopy image formation on a GaAs(110) surface: Effect of tip morphology," Phys. Rev. B 63, 245323.

Ke, S.-H., T. Uda, K. Terakura, R. Perez, and I. Stich, 2002, in Noncontact Atomic Force Microscopy, edited by S. Morita, R. Wiesendanger, and E. Meyer (Springer, Berlin), Chap. 16, pp. 279-304.

Kitamura, S., and M. Iwatsuki, 1995, "Observation of $7 \times 7$ reconstructed structure on the silicon(111) surface using ultrahigh vacuum noncontact atomic force microscopy," Jpn. J. Appl. Phys., Part 2 34, L145-L148.

Kitamura, S., and M. Iwatsuki, 1996, "Observation of silicon surfaces using ultrahigh-vacuum noncontact atomic force microscopy,” Jpn. J. Appl. Phys., Part 2 35, L668-L671.

Kitamura, S., and M. Iwatsuki, 1998, "High-resolution imaging of contact potential difference with ultrahigh-vacuum noncontact atomic force microscopy," Appl. Phys. Lett. 72, 31543156.

Kliewer, J., R. Berndt, and S. Crampin, 2000, "Controlled modification of individual adsorbate electronic structure," Phys. Rev. Lett. 85, 4936-4939.

Kobayashi, K., H. Yamada, H. Itoh, T. Horiuchi, and K. Matsushige, 2001, "Analog frequency modulation detector for dynamic force microscopy," Rev. Sci. Instrum. 72, 4383-4387.

Krupp, H., 1967, "Particle adhesion theory and experiment," Adv. Colloid Interface Sci. 1, 111-239.

Kuchling, H., 1982, Taschenbuch der Physik (Deutsch, Thun and Frankfurt/Main).

Kuk, Y., and P. J. Silverman, 1988, "Scanning tunneling microscope instrumentation," Rev. Sci. Instrum. 60, 165-180.

Landau, L., and E. M. Lifshitz, 1990, Lehrbuch der Theoretischen Physik I, 13th ed. (Akademie Verlag, Berlin).

Lantz, M., H. J. Hug, R. Hoffmann, P. van Schendel, P. Kappenberger, S. Martin, A. Baratoff, and H.-J. Güntherodt, 2001, "Quantitative measurement of short-range chemical bonding forces," Science 291, 2580-2583.

Lantz, M., H. J. Hug, P. Van Schendel, R. Hoffmann, S. Martin, A. Baratoff, A. Abdurixit, and H.-J. Güntherodt, 2000, "Low-temperature scanning force microscopy of the Si(111)(77) surface," Phys. Rev. Lett. 84, 2642-2645.

Law, B. M., and F. Rieutord, 2002, "Electrostatic forces in atomic force microscopy," Phys. Rev. B 66, 035402.

Livshits, A., A. Shluger, and A. Rohl, 1999, "Contrast mechanism in non-contact SFM images of ionic surfaces," Appl. Surf. Sci. 140, 327-332.

Livshits, A., A. Shluger, A. Rohl, and A. Foster, 1999, "Model of noncontact scanning force microscopy on ionic surfaces," Phys. Rev. B 59, 2436-2448.

Loppacher, C., R. Bennewitz, O. Pfeiffer, M. Guggisberg, M. Bammerlin, S. Schär, V. Barwich, A. Baratoff, and E. Meyer, 1998, "Phase variation experiments in non-contact dynamic force microscopy using phased-locked-loop techniques," Appl. Surf. Sci. 140, 287-292.

Loppacher, C., R. Bennewitz, O. Pfeiffer, M. Guggisberg, M. Bammerlin, S. Schär, V. Barwich, A. Baratoff, and E. Meyer,
2000, "Quantitative measurement of short-range chemical bonding forces," Phys. Rev. B 62, 13674-13679.

Lüthi, R., E. Meyer, M. Bammerlin, A. Baratoff, L. Howald, C. Gerber, and H.-J. Güntherodt, 1997, "Ultrahigh vacuum atomic force microscopy: True atomic resolution," Surf. Rev. Lett. 4, 1025-1029.

Lüthi, R., E. Meyer, M. Bammerlin, T. Lehmann, L. Howald, C. Gerber, and H.-J. Güntherodt, 1996, "Atomic resolution in dynamic force microscopy across steps on $\mathrm{Si}(111) 7 \times 7$," Z . Phys. B: Condens. Matter 100, 165-167.

Madelung, O., M. Schultz, and H. Weiss, Eds., 1982, Numerical Data and Functional Relationships in Science and Technology, Landolt-Börnstein, Vol. 17a (Springer, Berlin).

Manoharan, H., C. Lutz, and D. Eigler, 2000, "Quantum mirages formed by coherent projection of electronic structure," Nature (London) 403, 512-515.

Marcus, R., T. Ravi, T. Gmitter, K. Chin, D. Liu, W. Orvis, D. Ciarlo, C. Hunt, and J. Trujillo, 1990, "Formation of silicon tips with $1 \mathrm{~nm}$ radius," Appl. Phys. Lett. 56, 236-238.

Martin, Y., C. C. Williams, and H. K. Wickramasinghe, 1987, "Atomic force microscope-force mapping and profiling on a sub 100-^ scale,” J. Appl. Phys. 61, 4723-4729.

Mate, M., G. M. McClelland, R. Erlandsson, and C. Chiang, 1987, "Atomic-scale friction of a tungsten tip on a graphite surface," Phys. Rev. Lett. 59, 1942-1945.

McClelland, G. M., R. Erlandsson, and S. Chiang, 1987, "Atomic force microscopy: general principles and a new implementation," Rev. Prog. Quant. Nondestr. Eval. 6B, 1307-1314.

Meyer, E., H. Heinzelmann, D. Brodbeck, G. Overney, L. Howald, H. Hug, T. Jung, H.-R. Hidber, and H.-J. Güntherodt, 1990, "Atomic resolution on the surface of $\mathrm{LiF}(001)$ by atomic force microscopy," J. Vac. Sci. Technol. B 9, 1329-1332.

Meyer, E., H. Heinzelmann, H. Rudin, and H.-J. Güntherodt, 1990, "Atomic resolution on $\mathrm{LiF}(001)$ by atomic force microscopy,” Z. Phys. B: Condens. Matter 79, 3-4.

Meyer, G., and N. M. Amer, 1990, "Optical-beam-deflection atomic force microscopy: The $\mathrm{NaCl}(100)$ surface," Appl. Phys. Lett. 56, 2100-2101.

Meyer, G., S. Zöphel, and K.-H. Rieder, 1996, "Scanning tunneling microscopy manipulation of native substrate atoms: A new way to obtain registry information on foreign adsorbates," Phys. Rev. Lett. 77, 2113-2116.

Minobe, S. O. T., T. Uchihashi, Y. Sugawara, and S. Morita, 1999, "The atomic resolution imaging of metallic $\operatorname{Ag}(111)$ surface by noncontact atomic force microscopy," Appl. Surf. Sci. 140, 243-246.

Mody, C., 2002, "Probe Microscopists at Work and at Play: The Growth of American STM in the 1980s," unpublished.

Momosaki, E., 1997, Proceedings of the 1997 IEEE International Frequency Contr. Symposium (IEEE, New York), Vol. 56, pp. 552-565.

Morita, S., 2002, in Noncontact Atomic Force Microscopy, edited by S. Morita, R. Wiesendanger, and E. Meyer (Springer, Berlin), Chap. 1, pp. 1-10.

Morita, S., and Y. Sugawara, 2002, in Noncontact Atomic Force Microscopy, edited by S. Morita, R. Wiesendanger, and E. Meyer (Springer, Berlin), Chap. 3, pp. 47-78.

Morita, S., and M. Tsukada, 1999, "Proceedings of the First International Workshop on Noncontact Atomic Force Microscopy, Osaka, Japan, July 21-23, 1998," Appl. Surf. Sci. 140, 243-456. 
Morita, S., R. Wiesendanger, and E. Meyer 2002, Eds., Noncontact Atomic Force Microscopy, Nanoscience and Technology (Springer, Berlin).

Nakagiri, N., M. Suzuki, K. Okiguchi, and H. Sugimura, 1997, "Site discrimination of adatoms in $\mathrm{Si}(111)-7 \times 7$ by noncontact atomic force microscopy," Surf. Sci. Lett. 373, L329L332.

Ohnesorge, F., and G. Binning, 1993, "True atomic resolution by atomic force microscopy through repulsive and attractive forces," Science 260, 1451-1456.

Olsson, L., N. Lin, V. Yakimov, and R. Erlandsson, 1998, “A method for in situ characterization of tip shape in ac-mode atomic force microscopy using electrostatic interaction," $\mathrm{J}$. Appl. Phys. 84, 4060-4064.

Oral, A., R. A. Grimble, H. Özgur Özer, P. M. Hoffmann, and J. B. Pethica, 2001, "Quantitative atom-resolved force gradient imaging using noncontact atomic force microscopy," Appl. Phys. Lett. 79, 1915-1917.

Pang, C. L., and G. Thornton, 2002, in Noncontact Atomic Force Microscopy, edited by S. Morita, R. Wiesendanger, and E. Meyer (Springer, Berlin), Chap. 9, pp. 147-166.

Park, S. I., and R. C. Barrett, 1993, in Scanning Tunneling Microscopy, edited by J. A. Stroscio and W. J. Kaiser (Academic, Boston), Chap. 2, pp. 31-76.

Partin, J., 1995, “Atomic resolution of an insulator by noncontact AFM," Presentation at the 12th International Conference on Scanning Tunneling Microscopy 1995 (Snowmass, Colorado, USA).

Pauling, L., 1957, The Nature of the Chemical Bond (Cornell University, Ithaca, NY).

Perez, R., I. Stich, M. C. Payne, and K. Terakura, 1997, "Role of covalent tip-surface interactions in noncontact atomic force microscopy on reactive surfaces," Phys. Rev. Lett. 78, 678-681.

Perez, R., I. Stich, M. C. Payne, and K. Terakura, 1998, "Surface-tip interactions in noncontact atomic-force microscopy on reactive surfaces: Si(111)," Phys. Rev. B 58, 10 83510849.

Pethica, J. B., 1986, "Comment on 'Interatomic forces in scanning tunneling microscopy: Giant corrugations of the graphite surface,'” Phys. Rev. Lett. 57, 3235.

Pethica, J. B., and R. Egdell, 2001, "The insulator uncovered," Nature (London) 414, 27-29.

Pfeiffer, O., R. Bennewitz, A. Baratoff, and E. Meyer, 2002, "Lateral-force measurements in dynamic force microscopy," Phys. Rev. B 65, 161403(R).

Raza, H., C. L. Pang, S. A. Haycock, and G. Thornton, 1999,

"Evidence of concrete bond breaking steps in the $1 \times 1$ to 1 $\times 3$ phase transition of $\mathrm{TiO}_{2}(100)$," Phys. Rev. Lett. 82, 5265-5268.

Reichling, M., and C. Barth, 1999, "Scanning force imaging of atomic size defects on the $\mathrm{CaF}_{2}(111)$ surface," Phys. Rev. Lett. 83, 768-771.

Reichling, M., and C. Barth, 2002, in Noncontact Atomic Force Microscopy, edited by S. Morita, R. Weisendanger, and E. Meyer (Springer, Berlin), Chap. 6, pp. 109-124.

Rensen, W. H. J., N. F. van der Hulst, A. G. T. Ruiter, and P. E. West, 1999, "Atomic steps with tuning-fork-based noncontact atomic force microscopy," Appl. Phys. Lett. 75, 1640-1642.

Riordon, J., 2003, "Physical Review Letters' Top Ten. Number 4: Atomic force microscopy," APS News 12 (5), 3.

Rugar, D., and P. Hansma, 1990, "Atomic force microscopy," Phys. Today 43 (10), 23-30.
Ruiter, A., J. Veerman, K. O. van der Werf, and N. van Hulst, 1997, "Dynamic behavior of tuning fork shear-force feedback," Appl. Phys. Lett. 71, 28-30.

Rychen, J., T. Ihn, P. Studerus, A. Herrmann, and K. Ensslin, 1999, "A low-temperature dynamic mode scanning force microscope operating in high magnetic fields," Rev. Sci. Instrum. 70, 2765-2768.

Sarid, D., 1994, Scanning Force Microscopy, 2nd ed. (Oxford University Press, New York).

Sasahara, A., and H. Onishi, 2002, in Noncontact Atomic Force Microscopy, edited by S. Morita, R. Weisendanger, and E. Meyer (Springer, Berlin), Chap. 13, pp. 215-232.

Sasaki, N., and M. Tsukada, 1998, "The relation between resonance curves and tip-surface interaction potential in noncontact atomic-force microscopy," Jpn. J. Appl. Phys., Part 2 37, L533-L535.

Sasaki, N., and M. Tsukada, 1999, "Theory for the effect of the tip-surface interaction potential on atomic resolution in forced vibration system of noncontact AFM," Appl. Surf. Sci. 140, 339-343.

Sasaki, N., and M. Tsukada, 2000, "Effect of microscopic nonconservative process on noncontact atomic force microscopy," Jpn. J. Appl. Phys., Part 239 (12B), L1334-L1337.

Schiller, C., 2003, "Charakterisierung von STM/AFM Spitzen und atomar aufgelöste Messungen zum Abklingverhalten des Tunnelstroms," Diploma thesis (University of Augsburg) (English translation: "Characterization of STM/AFM tips and atomically resolved measurements of the decay characteristics of the tunneling current").

Schimmel, T., T., Koch, J. Küppers, and M. Lux-Steiner, 1999, "True atomic resolution under ambient conditions obtained by atomc force microscopy in the contact mode," Appl. Phys. A: Mater. Sci. Process. 68, 399-402.

Schirmeisen, A., G. Cross, A. Stalder, P. Grütter, and U. Dürig, 2000, "Metallic adhesion and tunnelling at the atomic scale," New J. Phys. 2, 29.1-29.10.

Schwarz, A., W. Allers, U. Schwarz, and R. Wiesendanger, 1999, "Simultaneous imaging of the In and As sublattice on InAs(110)-(1×1) with dynamic scanning force microscopy," Appl. Surf. Sci. 140, 293-297.

Schwarz, U. D., H. Hölscher, and R. Wiesendanger, 2001, in "Proceedings of the Third International Conference on NonContact Atomic Force Microscopy, Hamburg, Germany, July 16-19, 2000," Appl. Phys. A: Mater. Sci. Process. 72 (Supplement), S1-S141.

Shluger, A. L., L. N. Kantorovich, A. I. Livshits, and M. J. Gillan, 1997, "Ionic and electronic processes at ionic surfaces induced by atomic-force-microscope tips," Phys. Rev. B 56, $15332-15344$.

Shluger, A. L., A. I. Livshits, A. S. Foster, and C. R. A. Catlow, 1999, "Models of image contrast in scanning force microscopy on insulators," J. Phys.: Condens. Matter 11, R295-R322.

Smith, D. P. E., 1995, "Limits of force microscopy," Rev. Sci. Instrum. 66, 3191-3195.

Sokolov, I. Y., G. S. Henderson, and F. J. Wicks, 1999, "Pseudo-non-contact AFM imaging?" Appl. Surf. Sci. 140, 362-365.

Stillinger, F. H., and T. A. Weber, 1985, "Computer simulation of local order in condensed phases of silicon," Phys. Rev. B 31, 5262-5271.

Stroscio, J. A., and W. J. Kaiser, 1994, Eds., Scanning Tunneling Microscopy, 2nd ed. (Academic, Boston). 
Sugawara, Y., 2002, in Noncontact Atomic Force Microscopy, edited by S. Morita, R. Wiesendanger, and E. Meyer (Springer, Berlin), Chap. 11, pp. 183-192.

Sugawara, Y., M. Ohta, H. Ueyama, and S. Morita, 1995, "Defect motion on an $\operatorname{InP}(110)$ surface observed with noncontact atomic force microscopy," Science 270, 1646-1648.

Sugawara, Y., H. Ueyama, T. Uchihashi, M. Ohta, Y. Yanase, T. Shigematsu, M. Suzuki, and S. Morita, 1997, in Materials Research Society 1996 Fall Meeting (Boston, December 1996), Proceedings E: Defects in Electric Materials II, edited by J. Michel, T. Kennedy, K. Wada, and K. Thonke (Materials Research Society, Warrendale, PA), p. 16.

Tabor, D., and R. H. S. Winterton, 1969, "Direct measurement of normal and retarded van der Waals forces," Proc. R. Soc. London, Ser. A 312, 435-450.

Takayanagi, K., Y. Tanishiro, M. Takahashi, and S. Takahashi, 1985, "Structural analysis of $\mathrm{Si}(111)-7 \times 7$ by UHVtransmission electron diffraction and microscopy," J. Vac. Sci. Technol. A 3, 1502-1506.

Tobik, J., I. Stich, and K. Terakura, 2001, "Effect of tip morphology on image formation in noncontact atomic force microscopy: InP(110)," Phys. Rev. B 63, 245324.

Todorovic, M., and S. Schulz, 1998, "Magnetic force microscopy using nonoptical piezoelectric quartz tuning fork detection design with applications to magnetic recording studies," J. Appl. Phys. 83, 6229-6231.

Tomlinson, G. A., 1929, "A molecular theory of friction," Philos. Mag. 7, 905-939.

Tortonese, M., R. C. Barrett, and C. Quate, 1993, “Atomic resolution with an atomic force microscope using piezoresistive detection," Appl. Phys. Lett. 62, 834-836.

Tsai, D. P., and Y. Y. Lu, 1998, "Tapping-mode tuning fork force sensing for near-field scanning optical microscopy," Appl. Phys. Lett. 73, 2724-2726.

Tsukada, M., and S. Morita, 2002, in "Proceedings of the Fourth International Conference on Noncontact Atomic
Force Microscopy, Kyoto, Japan, September 21-23, 2001," Appl. Surf. Sci. 188, 231-554.

Tsukada, M., N. Sasaki, M. Gauthier, K. Tagami, and S. Watanabe, 2002, in Noncontact Atomic Force Microscopy, edited by S. Morita, R. Wiesendanger, and E. Meyer (Springer, Berlin), Chap. 15, pp. 257-278.

Ueyama, H., Y. Sugawara, and S. Morita, 1998, "Stable operation mode for dynamic noncontact atomic force microscopy," Appl. Phys. A: Mater. Sci. Process. 66(Supplement), S295S297.

Walls, F. L., 1985, in Precision Frequency Control, edited by E. Gerber and A. Ballato (Academic, Orlando, FL), pp. 276279.

Wang, L., 1998, "Analytical descriptions of the tapping-mode atomic force microscopy response," Appl. Phys. Lett. 73, 3781-3783.

Wickramasinghe, H. K., 1989, "Scanned-Probe Microscopes," Sci. Am. 261 (4), 74-81.

Wiesendanger, R., 1994, Scanning Probe Microscopy and Spectroscopy: Methods and Applications (Cambridge University Press, Cambridge, UK).

Wiesendanger, R., 1998, Scanning Probe Microscopy: Analytical Methods (Springer, Berlin).

Wolter, O., T. Bayer, and J. Greschner, 1991, "Micromachined silicon sensors for scanning force microscopy," J. Vac. Sci. Technol. A 9, 1353-1357.

Yamada, H., 2002, in Noncontact Atomic Force Microscopy, edited by S. Morita, R. Wiesendanger, and E. Meyer (Springer, Berlin), Chap. 12, pp. 193-214.

Yokoyama, K., T. Ochi, Y. Sugawara, and S. Morita, 1999, "Atomically resolved silver imaging on the $\operatorname{Si}(111)-(\sqrt{3}$ $\times \sqrt{3})$ - Ag surface using a noncontact atomic force microscope," Phys. Rev. Lett. 83, 5023-5026.

Zhong, Q., D. Innis, K. Kjoller, and V. B. Elings, 1993, "Fractured polymer silica fiber surface studied by tapping mode atomic-force microscopy," Surf. Sci. 290, L688-L692. 